\title{
IRREDUCIBILITY OF SOME UNITARY REPRESENTATIONS OF THE POINCARÉ GROUP WITH RESPECT TO THE POINCARÉ SUBSEMIGROUP, III
}

\author{
HITOSHI KANETA
}

The aim of this paper is to prove that irreducible unitary representations $\left(U^{\ell, \varepsilon}, \mathfrak{F}_{\mathcal{C}}^{\ell, \varepsilon}\right)$ of the Poincaré group $P=R^{4} \times{ }_{s} S L(2, C)$ are reducible as the representations of the Poincaré subsemigroup $P_{+}=V_{+} \times_{s} S L(2, C)$ with $V_{+}=\left\{x_{0}^{2}-x_{2}^{2}-x_{3}^{2} \geq 0, x_{0} \geq 0\right\}$. The representations mentioned above are those associated with the one-sheeted hyperboloid $V_{i M}=\left\{y_{0}^{2}-y_{1}^{2}-y_{2}^{2}\right.$ $\left.-y_{3}^{2}=-M^{2}\right\}(M>0)$ and the irreducible unitary representations $\pi_{(\ell, \varepsilon)}$ of $S U(1,1)$ not belonging to the discrete series (see the end of this introduction for the definition of the discrete series). To attain our purpose we shall determine all $P_{+}$-invariant, closed proper subspaces for the representations $\left(U^{\ell, \epsilon}, \mathfrak{S}^{\ell, \varepsilon}\right)$ (Theorems 1.1 and 4.1). Other irreducible unitary representations of $P$ are known to be irreducible even when they are restricted to $P_{+}[6]$.

In [6], [7] and this paper we are concerned with the question whether (Q) there exists a $P_{+}$-invariant, closed proper subspace for an irreducible unitary representation of $P$.

A physical aspect of this problem is as follows. From E. Wigner's view point of relativistic quantum mechanics an irreducible unitary representation $(U, \mathfrak{F})$ describes the dynamics of an elementary particle. In particular the one-parameter unitary group $U(t, 0,0,0, e)(t \in R)$ on $\mathscr{F}$ stands for the dynamical transformation group. On the other hand some elementary particles (a neutral pion, for example) are known to decay spontaneously. If one tries to explain the phenomena from Wigner's point of view, one naturally expects that there exists a proper closed subspace $\mathscr{D}$ of $\mathfrak{S}$ such that $\mathscr{D}$ is invariant under $U(t, 0,0,0, e)(t \geq 0)$ and $U(0,0,0,0, g)$ $\left(g \in S L(2, C)\right.$ ), equivalently such that $\mathscr{D}$ is $P_{+}$-invariant. We are very likely to suspect the existence of an irreducible unitary representation of $P$ with

Received November 29, 1980. 
this property. In reality, however, there do exist such representations.

As in [7] the Hilbert transform and the Frobenius method for ordinary differential equations with a regular singularity find their applications here too. But the most effective measures are provided by the eigenfunction expansion theorems in $[4,5]$. This is because we must deal with second and first order ordinary differential operators $L_{k, \ell}$ and $M_{k, \ell}$ respectively, acting on $L^{2}(R)^{2 k+1}$ (see $(1,13),(1,14)$ ). Of course these operators are connected with the Laplacians $\Delta$ and $\Delta^{\prime}$ of $S L(2, C)$ respectively.

In $\S 1$, after the definition of the representation $\left(U^{\ell, \varepsilon}, \mathfrak{F}_{\mathcal{E}}^{\ell, s}\right)$ we shall show that, if the statement $(Q)$ above is valid for this representation, there exists a non-trivial sequence $\left\{D_{k}\right\}_{k \in Z_{++}}$of closed subspaces in $L^{2}(R)_{2 k+1}$ or $L^{2}(R)$ such that it satisfies certain conditions (Q.1) and (Q.2) in Lemma 1.4. Conversely, once such sequences are given (Proposition 1.5, Theorems 2.2 and 3.1), we can construct $P_{+}$-invariant subspaces $\mathscr{D}_{ \pm}^{\ell, e}$ of $\mathfrak{S}_{\mathcal{C}}^{\ell, s}$ (Theorems 1.1 and 4.1) mainly due to Proposition 1.6. To determine all nontrivial sequences $\left\{D_{k}\right\}_{k \in Z_{++}}$satisfying the conditions (Q.1) and (Q.2) is, therefore, the core of our argument. The simplest case, in which $\pi_{(\ell, \varepsilon)}$ is the unit representation of $S U(1,1)$, namely $(\ell, \varepsilon)=(0,0)$, is discussed in $\S 1$, while the other cases are investigated in $\S \S 2$ and 3 . In the final section, $\S 4$, we shall describe all the $P_{+}$-invariant, closed proper subspaces of $\mathfrak{S}^{\ell, s}$ for $(\ell, \varepsilon) \neq(0,0)$.

Notation and terminology.

$Z$ is the set of integers and $Z_{+}=\{n \in Z ; n \geq 0\}$.

$R$ is the set of real numbers, $R_{+}=\{\lambda \in R ; \lambda>0\}$ and $R^{*}=R \backslash\{0\}$.

$C$ is the set of complex numbers and $C^{*}=C \backslash\{0\} . \quad T=\{z \in C ;|z|=1\}$. $D_{\tau}=\left\{z \in C ;|\operatorname{Im} z|<\pi / 2, \bar{D}_{\tau}=\{z \in C ;|\operatorname{Im} z| \leq \pi / 2\}\right.$ and $\dot{D}_{\tau}=\bar{D}_{\tau} \mid\{ \pm i \pi / 2\}$. Throughout this paper $\sigma=\tau-i \pi / 2 . \quad V_{i M}=\left\{y \in R^{4} ; y_{0}^{2}-y_{1}^{2}-y_{2}^{2}-y_{3}^{2}=-M^{2}\right\}(M>0)$ and $B_{3}=R \times(0, \pi) \times(0,2 \pi) . \quad R_{k} f(\sigma)={ }^{t}\left(f_{-k}(-\sigma), \cdots, f_{k}(-\sigma)\right)$ for a function $f(\sigma)={ }^{t}\left(f_{k}(\sigma), \cdots, f_{-k}(\sigma)\right), k \in Z_{+} / 2$. A polynomial in $\log \sigma$ with holomorphic coefficients will be denoted by $h(\sigma, \log \sigma)$, namely $h(\sigma, \log \sigma)=\sum_{n} h_{n}(\sigma) \times$ $(\log \sigma)^{n}$, where $h_{n}(\sigma)$ are holomorphic in a vicinity of zero. $G_{\alpha}=(\alpha-i \operatorname{sh} \tau)^{-1}$ $(\operatorname{Re} \alpha>0)$. For the definition of the function $P_{\mu \nu}^{k}(z)$, see [13, p. 120]. We abbreviate the integral $\int_{R} f(\tau) d \tau$ to $\int f d \tau$ or $\langle f\rangle . \quad a \propto b$ means $a=c b$ for some $c \in C^{*} .((a \pm b))=(a+b)(a-b)$, and $\operatorname{sign} \nu= \pm 1$ if $\pm \nu>0$. $M_{m, n}$ is the set of all complex $m \times n$-matrices, $M_{n}=M_{n, n}, M_{n}^{+}=\left\{A \in M_{n}\right.$; $A \geq 0\}$ and $M_{n}^{++}=\left\{A \in M_{n} ; A>0\right\} . \quad I_{n}$ stands for the unit matrix in $M_{n}$. 
For $A=\left(a_{j k}\right)$ in $M_{m, n}$ denote by ${ }^{t} A=\left(a_{k j}\right)$ the transposed matrix of $A$ and set $\bar{A}=\left(\bar{a}_{j k}\right), A^{*}={ }^{t} \bar{A}$ and $|A|=\max _{k} \sum_{j}\left|a_{j k}\right|$.

$C^{r}(S)^{n}(r=0,1, \cdots, \infty)$ for a $C^{\infty}$-manifold $S$ is the totality of $C^{n}$-valued $C^{r}$-functions on $S . \quad C_{0}^{r}(S)^{n}=f \in C^{r}(S)^{n} ; f$ is compactly supported $\} . \quad C_{0}^{0}(S)^{n}$ $=C_{0}(S)^{n} . \quad H_{r}(R)\left(r \in Z_{+}\right)$is the Sobolev space of order $r$ on $R . H_{r}(R)^{n}=$ $\sum_{j=1}^{n} \oplus H_{r}(R)$ and $L^{2}(R)^{n}=H_{0}(R)^{n}$. Let $(B, \Sigma)$ be a measurable space, where $B$ is a Borel subset of $R^{n}$ and $\Sigma$ is the set of all Borel sets in $B$. $L^{2}(B, \mu)$ is the usual $L^{2}$-space defined in terms of the measure $\mu$ on $(B, \Sigma)$. Let $\rho$ be a $M_{n}^{++}$-valued Borel measurable function on $B$. $L^{2}(B, \rho)=$ $L^{2}(B, \rho d x)$ stands for the Hilbert space consisting of $C^{n}$-valued Borel measurable functions $f$ on $B$ such that $\int_{B} f^{*}(x) \rho(x) f(x) d x<\infty$, where $d x$ is the Lebesgue measure. a.e. means almost everywhere with respect to the Lebesgue measure.

Let $L$ be a linear operator $L: H_{1} \rightarrow H_{2}$. Then $\operatorname{Ker} L$ is the kernel of $L$. When both $H_{j}$ are Hilbert spaces, $L^{*}$ means the (formal) adjoint of $L$. $L H_{1}$ denotes the range of $L$, namely $\left\{L h ; h \in H_{1}\right.$ lies in the domain of $\left.L\right\}$. Let $H_{0}$ be a subspace of $H_{2}$. Then $L \mid H_{0}$ denotes the restriction of $L$ to $H_{0}$. $D^{\perp}=\{h \in H ; h$ is orthogonal to $D\}$ for a Hilbert space $H$ and its subset $D$. $\langle$,$\rangle and \|\|$ stand for the inner product and the norm on a Hilbert space $\left(C^{n}, L^{2}(B, \mu)\right.$, etc.) respectively. However, $\langle x, y\rangle=x_{0} y_{0}-x_{1} y_{1}-x_{2} y_{2}$ $-x_{3} y_{3}$ for $x, y$ in $R^{4}$ and $\langle f\rangle=\int_{R} f(\tau) d \tau$ for an integrable function $f$ on $R$. Throughout this paper Hilbert spaces are understood to be separable. $G=S L(2, C), G_{0}=S U(1,1)=\left\{\left(\frac{\alpha}{\beta} \frac{\beta}{\alpha}\right) ;|\alpha|^{2}-|\beta|^{2}=1\right\}$ and $P=R^{4} \times{ }_{s} S L(2, C)$ with the multiplication $(x, g)\left(x^{\prime}, g^{\prime}\right)=\left(x+g^{*-1} x^{\prime} g^{-1}, g g^{\prime}\right)$, where any $x=$ $\left(x_{0}, x_{1}, x_{2}, x_{3}\right)$ in $R^{4}$ is identified with the matrix $\left(\begin{array}{ll}x_{0}-x_{3} & x_{2}-i x_{1} \\ x_{2}+i x_{1} & x_{0}+x_{3}\end{array}\right)$.

$\varsigma_{m, \rho}$ are irreducible unitary representations of $G$ belonging to the continuous series $[12, \S 11]$. $\pi_{(\ell, s)}$ stand for irreducible unitary representations of $G_{0}$ (see the beginning of $\S 1$ ). One-parameter subgroups $\omega_{j}(t)$, $1 \leq j \leq 6$, of $G$ are given as follows.

$$
\begin{aligned}
& \omega_{1}(t)=\left(\begin{array}{rr}
\cos t / 2 & i \sin t / 2 \\
i \sin t / 2 & \cos t / 2
\end{array}\right), \quad \omega_{2}(t)=\left(\begin{array}{rr}
\cos t / 2 & -\sin t / 2 \\
\sin t / 2 & \cos t / 2
\end{array}\right), \\
& \omega_{3}(t)=\left(\begin{array}{cc}
\exp i t / 2 & 0 \\
0 & \exp -i t / 2
\end{array}\right), \quad \omega_{4}(t)=\left(\begin{array}{cc}
\operatorname{ch} t / 2 & \operatorname{sh} t / 2 \\
\operatorname{sh} t / 2 & \operatorname{ch} t / 2
\end{array}\right), \\
& \omega_{5}(t)=\left(\begin{array}{rr}
\operatorname{ch} t / 2 & i \operatorname{sh} t / 2 \\
-i \operatorname{sh} t / 2 & \operatorname{ch} t / 2
\end{array}\right), \quad \omega_{6}(t)=\left(\begin{array}{cc}
\exp t / 2 & 0 \\
0 & \exp -t / 2
\end{array}\right) .
\end{aligned}
$$

$(\tau, \theta, \phi)$ in $B_{3}=R \times(0, \pi) \times(0,2 \pi)$ is a local coordinate of a open dense 
subset of $V_{i M}=\left\{y \in R^{4} ; y_{0}^{2}-y_{1}^{2}-y_{2}^{2}-y_{3}^{2}=-M^{2}\right\}(M>0)$ in the sense that the map $(\tau, \theta, \phi) \rightarrow\left(\omega_{6}(\tau) \omega_{2}(\theta) \omega_{3}(\phi)\right) * \hat{y} \omega_{6}(\tau) \omega_{2}(\theta) \omega_{3}(\phi)$ of $B_{3}$ into $V_{i M}$ is a diffeomorphism, where $\hat{y}=M\left(\begin{array}{rr}-1 & 0 \\ 0 & 1\end{array}\right)$.

Let $(T, \mathfrak{S})$ be a continuous unitary representation of $G$. Then, we set

$$
\begin{aligned}
& \omega_{j}=d /\left.d t\right|_{t=0} T\left(\omega_{j}(t)\right)(1 \leq j \leq 6), H_{ \pm}=i \omega_{2} \pm \omega_{1}, H_{3}=i \omega_{3}, \\
& F_{ \pm}=i \omega_{5} \pm \omega_{4}, F_{3}=i \omega_{6}, \Delta_{0}=-\left(H_{+} H_{-}+H_{-} H_{+}+2 H_{3}^{2}\right) / 2, \\
& \Delta=\left(F_{+} F_{-}+F_{-} F_{+}+2 F_{3}^{2}\right) / 2+\Delta_{0}-1, \\
& \Delta^{\prime}=\left(H_{+} F_{-}+H_{-} F_{+}+F_{+} H_{-}+F_{-} H_{+}+4 H_{3} F_{3}\right) / 2 .
\end{aligned}
$$

A closed subspace $D$ of a Hilbert space $H$ is said to be invariant under a self-adjoint operator $L$ if $P_{D} L=L P_{D}$, where $P_{D}$ is the orthogonal projection: $H \rightarrow D$.

An irreducible unitary representation $\left(\pi, \mathscr{F}_{\pi}\right)$ of $G_{0}$ is said to belong to the discrete series in our sense if the selfadjoint operator $d /\left.d t\right|_{t=0} i \pi\left(\omega_{3}(t)\right)$ is unbounded, but bounded either from below or above.

\section{§1. $P_{+}$-invariant subspaces for the representation $\left(U^{0,0}, \mathscr{S}^{0,0}\right)$}

After defining irreducible unitary representations $\left(U^{\ell, \varepsilon}, \mathfrak{S}^{\ell, \varepsilon}\right)$ of $P$ associated with the one-sheeted hyperboloid $V_{i M}$ and irreducible unitary representations $\pi_{(\ell, \varepsilon)}$ of $G_{0}$, we shall obtain all the $P_{+}$-invariant, closed prober subspaces in $\mathfrak{S}_{\mathcal{C}}^{0,0}$. Here $\pi_{(0,0)}$ stands for the unit representation while $\pi_{(\ell, \varepsilon)}((\ell, \varepsilon) \neq(0,0))$ stands for the irreducible one $T_{(\ell, \varepsilon)}$ not belonging to the discrete series [13, p. 305]. Thus $\pi_{(-1 / 2+i \eta, 1 / 2)}(\eta>0), \pi_{(-1 / 2+i \eta, 0)}(\eta \geq 0)$ and $\pi_{(\ell, 0)}(-1<\ell<-1 / 2)$ are irreducible representations belonging to the continuous spinor series, the continuous non-spinor series and the supplementary series respectively. $P_{+}$-invariant subspaces in $\mathfrak{S}^{\ell, \varepsilon}((\ell, \varepsilon) \neq(0,0))$ will be discussed in $\S 4$, since it is necessary to determine nontrivial sequences $\left\{D_{k}\right\}_{k \in Z_{++}}$which satisfy certain conditions (Q.1) and (Q.2) in advance. See Lemma 1.4 for the definition of (Q.1) and (Q.2).

Let $G$ act on $R^{4}$ by $y \cdot g=g^{*} y g$, where $y=\left(y_{0}, y_{1}, y_{2}, y_{3}\right)$ is identified with the matrix $\left(\begin{array}{ll}y_{0}-y_{3} & y_{2}-i y_{1} \\ y_{2}+i y_{1} & y_{0}+y_{3}\end{array}\right)$. Then the isotropy group at $\hat{y}=M$ $\times\left(\begin{array}{rr}-1 & 0 \\ 0 & 1\end{array}\right)$ is $G_{0}=S U(1,1)$, and a map $p: G \rightarrow V_{i M}$ defined by $p(g)=g^{*} \hat{y} g$ is a surjection. We fix once for all measurable sections $s_{u}(u \in S U(2))$ such that $p \circ s_{u}$ is the identity and that

$$
s_{u} \circ p(\langle\tau, \theta, \phi\rangle)=\langle\tau, \theta, \phi\rangle u \text { for }(\tau, \theta, \phi) \in B_{3}=R \times(0, \pi) \times(0,2 \pi),
$$

where $\langle\tau, \theta, \phi\rangle=\omega_{6}(\tau) \omega_{2}(\theta) \omega_{3}(\phi)$. Denote by $\mathfrak{F}_{\pi}$ and $d y_{1} d y_{2} d y_{3} / M^{2}\left|y_{0}\right|$ the 
representation space of $\pi=\pi_{(\ell, \ell)}$ and a $G$-invariant measure on $V_{i M}$ respectively. Following Mackey [10], we can define irreducible unitary representations $\left(U^{\pi, u}, \mathfrak{S C}^{\pi}\right)$ associated with $V_{i M}$ and $\pi$ as follows;

$$
\begin{aligned}
& \mathfrak{S}^{\pi}=L^{2}\left(V_{i M} \rightarrow \mathscr{S}_{\pi}, d y_{1} d y_{2} d y_{3} / M^{2}\left|y_{0}\right|\right), \\
& {\left[U^{\pi, u}(x, g) F\right](y)=e^{-i\left\langle x^{\prime}, \hat{y}\right\rangle} \pi\left(g_{0}\right) F(y \cdot g),}
\end{aligned}
$$

where $F \in \mathfrak{S}^{\pi}$ and $\left(0, s_{u}(y)\right)(x, g)=\left(x^{\prime}, g_{0}\right)\left(0, s_{u}(y \cdot g)\right)$ with $g_{0} \in G_{0}$. Of course $\mathfrak{S}_{\mathrm{C}^{\pi}}$ denotes a Hilbert space consisting of the square integrable $\mathfrak{S}_{\pi}$-valued functions on $V_{i M}$ with respect to the measure $d y_{1} d y_{2} d y_{3}\left|M^{2}\right| y_{0} \mid$. Since the image $\left\{p(\langle\tau, \theta, \phi\rangle) ;(\tau, \theta, \phi) \in B_{3}\right\}$ is dense and open in $V_{i M}$, we can naturally identify $\mathfrak{S}^{\pi}$ with a Hilbert space $\mathfrak{S}^{\ell, \varepsilon}$;

$$
\mathfrak{S C}_{\mathfrak{C}}^{\ell, \varepsilon}=L^{2}\left(B_{3} \rightarrow \mathfrak{S}_{\pi}, \operatorname{ch}^{2} \tau \sin \theta d \tau d \theta d \phi\right)
$$

which is, by definition, a Hilbert space consisting of square integrable $\mathfrak{S}_{\pi}$-valued functions on $B_{3}$ relative to $\operatorname{ch}^{2} \tau \sin \theta d \tau d \theta d \phi$. Under this identification $\left(U^{\pi, e}, \mathfrak{S}^{\pi}\right)$ gives a representation $\left(U^{\ell, \varepsilon}, \mathfrak{S}^{\ell, \varepsilon}\right)$ which we intended to define. Trivially $\mathfrak{S}_{\mathrm{C}}^{0,0}=L^{2}\left(B_{3}, \operatorname{ch}^{2} \tau \sin \theta d \tau d \theta d \phi\right)$ while $C_{0}^{\infty}\left(B_{3} \times T\right)$ is dense in $\mathfrak{S}_{\mathrm{C}}{ }^{\ell, \varepsilon}$ provided $(\ell, \varepsilon) \neq(0,0)$. In the latter case we have, for $f \in C_{0}^{\infty}\left(B_{3} \times T\right)$,

$$
\begin{aligned}
& {\left[U^{\ell, \varepsilon}(0, g) f\right]\left(\tau, \theta, \phi, e^{i \psi}\right)} \\
& \quad=\left(\beta e^{i \psi}+\bar{\alpha}\right)^{\ell+\varepsilon}\left(\bar{\beta} e^{-i \psi}+\alpha\right)^{\ell-\varepsilon} f\left(\tau^{\prime}, \theta^{\prime}, \phi^{\prime}, \frac{\alpha e^{i \psi}+\bar{\beta}}{\beta e^{i \psi}+\bar{\alpha}}\right),
\end{aligned}
$$

where $\langle\tau, \theta, \phi\rangle g=\left(\begin{array}{cc}\frac{\alpha}{\beta} & \frac{\beta}{\alpha}\end{array}\right)\left\langle\tau^{\prime}, \theta^{\prime}, \phi^{\prime}\right\rangle$. It also follows from (1.1) that

$$
U^{\ell, s}(t, 0,0,0, e)=\exp \{i M t \operatorname{sh} \tau\} .
$$

Now regarding $\left(U^{\ell, \varepsilon}, \mathfrak{S}_{\mathcal{C}}^{\ell, \varepsilon}\right)$ as a representation of $G$, we define operators $\omega_{j}(1 \leq j \leq 6), H_{ \pm}, H_{3}, F_{ \pm}, F_{3}, \Delta_{0}, \Delta$ and $\Delta^{\prime}$. As to the domains of these operators, see [6, p. 117]. In the case $(\ell, \varepsilon) \neq(0,0)$ explicit forms of these operators restricted to $C_{0}^{\infty}\left(B_{3} \times T\right)$ are known [6, §4] except for that of $\Delta$ (there is a misprint on p. 122, namely $p(g)=-g^{*} \hat{x} g$ instead of $g^{*} \hat{x} g$ ). Painstaking calculation is necessary to derive the following formula. See [6, p. 127] for the explicit form of $\Delta_{0}$.

$$
\begin{aligned}
\Delta= & -\partial_{\tau}^{2}-\operatorname{th}^{2} \tau \partial_{\theta}^{2}-\frac{2 \operatorname{th} \tau}{\operatorname{ch} \tau} \sin \psi \partial_{\theta} \partial_{\psi}-\frac{\operatorname{th}^{2} \tau}{\sin ^{2} \theta} \partial_{\phi}^{2} \\
& +\frac{2 \operatorname{th} \tau}{\sin \theta}\left\{\operatorname{th} \tau \cot \theta+\frac{\cos \psi}{\operatorname{ch} \tau}\right\} \partial_{\phi} \partial_{\psi}
\end{aligned}
$$




$$
\begin{aligned}
& -\left\{\operatorname{th}^{2} \tau \cot ^{2} \theta+\frac{2 \operatorname{th} \tau \cot \theta \cos \psi}{\operatorname{ch} \tau}+\frac{1}{\operatorname{ch}^{2} \tau}\right\} \partial_{\psi}^{2}-2 \operatorname{th} \tau \partial \\
& +\left\{\frac{2 \operatorname{th} \tau}{\operatorname{ch} \tau}(\ell \cos \psi+\varepsilon i \sin \psi)-\operatorname{th}^{2} \tau \cos \theta\right\} \partial_{\theta} \\
& -\frac{2 i \operatorname{th} \tau}{\operatorname{ch} \tau \sin \theta}\{\ell i \sin \psi+\varepsilon(\cos \psi+\varepsilon \operatorname{sh} \tau \cot \theta)\} \partial_{\phi} \\
& +\frac{2}{\operatorname{ch}^{2} \tau}\left\{-\ell \operatorname{sh} \tau \cot \theta \sin \psi+\varepsilon i\left(\operatorname{sh}^{2} \tau \cot ^{2} \theta\right.\right. \\
& +2 \operatorname{sh} \tau \cot \theta \cos \psi+1)\} \partial_{\psi}-\frac{\ell^{2}+\ell-\varepsilon^{2}}{\operatorname{ch}^{2} \tau} \\
& +\varepsilon^{2}\left(\operatorname{th} \tau \cot { }^{2} \theta+\frac{2 \operatorname{th} \tau}{\operatorname{ch} \tau} \cot \theta \cos \psi\right) \\
& +2 \varepsilon \ell i \frac{\operatorname{th} \tau}{\operatorname{ch} \tau} \cot \theta \sin \psi-1+\Delta_{0} .
\end{aligned}
$$

In the case $(\ell, \varepsilon)=(0,0)$ explicit forms of $H_{ \pm}, H_{3}$, etc. restricted to $C_{0}^{\infty}\left(B_{3}\right)$ take the forms;

$$
\begin{aligned}
H_{ \pm} & =e^{\mp i \phi}\left(i \partial_{\theta} \pm \cot \theta \partial_{\phi}\right), \quad H_{3}=i \partial_{\phi}, \\
F_{ \pm} & =e^{\mp i \phi}\left(\mp \sin \theta \partial_{\tau} \mp \operatorname{th} \tau \cos \theta \partial_{\theta}+i \frac{\operatorname{th} \tau}{\sin \theta} \partial_{\phi}\right), \quad \Delta^{\prime}=0, \\
(1.6) \quad{ }^{\prime} & =i\left(\cos \theta \partial_{\tau}-\operatorname{th} \tau \sin \theta \partial_{\theta}\right), \quad \Delta_{0}=\partial_{\theta}^{2}+\cot \theta \partial_{\theta}+\frac{1}{\sin ^{2} \theta} \partial_{\phi}^{2}, \\
\Delta & =-\partial_{\tau}^{2}-2 \operatorname{th} \tau \partial_{\tau}-\operatorname{th}^{2} \tau\left(\partial_{\theta}^{2}+\cot \theta \partial_{\theta}+\frac{1}{\sin ^{2} \theta} \partial_{\phi}^{2}\right)-1+\Delta_{0} .
\end{aligned}
$$

Notice that (1.6) follows from the corresponding ones in the case $(\ell, 0)$, $\ell \neq 0$, simply by deleting terms containing functions of $\psi$ or $\partial_{\psi}$ and setting $\ell=0$. Put, for $k \in Z_{+} / 2$ and $\mu=-k,-k+1, \cdots, k$,

$$
\mathscr{W}_{k, \mu}^{\ell, \varepsilon}=\left\{f \in \mathfrak{S}^{\ell, s} ; \Delta_{0} f=-k(k+1) f, H_{3} f=\mu f\right\} .
$$

Then, as is well-known, $\mathfrak{S}^{\ell, \varepsilon}=\sum_{k, \mu} \oplus \mathscr{W}_{k, \mu}^{\ell, \varepsilon}$.

Proposition 1.1. $\mathscr{W}_{k, \mu}^{\ell, \varepsilon}=\{0\}$ if $k+\varepsilon \in Z_{+}+1 / 2$. Otherwise,

$$
\begin{aligned}
& \mathscr{W}_{k, \mu}^{0,0}=\left\{f(\tau) P_{\mu, 0}^{k}(\cos \theta) e^{-i \mu \phi} ; f \in L^{2}\left(R, \operatorname{ch}^{2} \tau\right)\right\}, \\
& \mathscr{W}_{k, \mu}^{\ell, \varepsilon}=\left\{\sum_{\nu=-k}^{k} f_{\nu}(\tau) P_{\mu,-\nu}^{k}(\cos \theta) e^{-i \mu \phi+i(\nu+\varepsilon) \psi} ; f_{\nu} \in L^{2}\left(R, \operatorname{ch}^{2} \tau\right)\right\}
\end{aligned}
$$

for $(\ell, \varepsilon) \neq(0,0)$. See [13, p. 120] for the definition of $P_{\mu \nu}^{k}(z)$. 
Proof. Suppose $f_{1} \in C_{0}^{\infty}(R)$. Then $f_{1} P_{\mu, 0}^{k} e^{-i \mu \phi}\left(k \in Z_{+}\right)$or $f_{1} P_{\mu,-\nu}^{k} e^{-i \mu \phi+i(\nu+\varepsilon) \psi}$ $\left(k+\varepsilon \in Z_{+}\right)$lies in the domains of $\omega_{j}, \Delta_{0}, \Delta$ and $\Delta^{\prime}$, and these operators act on these functions as smooth differential operators. These facts can be shown as Lemma 9 [6]. After this remark we shall prove the proposition only in the case $\mu=k$, for the other cases can be dealt with by the aid of the relation $\mathscr{W}_{k, \mu}^{\ell, s}=H_{-}^{k-\mu} \mathscr{W}_{k, k}^{\ell, s}$ and a formula [13, p. 137];

$$
\left(\sqrt{1-z^{2}} \frac{d}{d z}-\frac{\mu z-\nu}{1-z^{2}}\right) P_{\mu \nu}^{k}(z)=-i \sqrt{(k+\mu)(k-\mu+1)} P_{\mu-1, \nu}^{k}(z) .
$$

Thanks to Proposition 1 [6] it is enough to consider the case $(\ell, \varepsilon)=(0,0)$. First since $U^{0,0}(0,-e)$ is the identity operator, there results that $\mathscr{W}_{k, \mu}^{0,0}=\{0\}$ if $k \notin Z_{+}$. Secondly, it is easily seen that $f_{1} P_{k, 0}^{k} e^{-i k \phi} \in \mathscr{W}_{k, k}^{0,0}\left(k \in Z_{+}\right)$, for $P_{k, 0}^{k}$ is a solution of the following equation.

$$
\begin{array}{r}
\left\{\left(1-z^{2}\right) d^{2} / d z^{2}-2 z d / d z-k^{2} /\left(1-z^{2}\right)+k(k+1)\right\} Q(z)=0 \\
z \in(-1,1)
\end{array}
$$

Finally assume $f \in \mathscr{W}_{k, k}^{0,0}\left(k \in Z_{+}\right)$. Let $h(\tau, \theta, \phi)=h_{1}(\tau) h_{2}(\theta) h_{3}(\phi)$ be an element of $C_{0}^{\infty}\left(B_{3}\right)$. Since $\left\langle f,\left(H_{3}-k\right) h\right\rangle=0$, there results $f=f_{2} e^{-i k \dot{\phi}}$ for an $f_{2} \in L^{2}\left(R \times(0, \pi), \operatorname{ch}^{2} \tau \sin \theta\right)$. Now the equality $\left\langle f,\left\{\Delta_{0}+k(k+1)\right\} h\right\rangle=0$ yields

$$
\left\langle f_{2},\left\{\partial_{\theta}^{2}+\cot \theta \partial_{\theta}-k^{2} / \sin ^{2} \theta+k(k+1)\right\} h_{1} h_{2}\right\rangle\left\langle e^{-i k \phi}, h_{3}\right\rangle=0 .
$$

Set $f_{2}(\tau, \theta)=g(\tau, \cos \theta)$. Then $g(\tau, \cdot)$ is a weak solution of (1.9) for a.e. $\tau$. Consequently there are measurable functions $f_{1, j}(\tau)$ such that $f_{2}(\tau, \theta)=$ $\sum_{j=1}^{2} f_{1, j} Q_{j}(\cos \theta)$ a.e. on $R \times(0, \pi)$, where $Q_{j}$ is a fundamental system of (1.9) with $Q_{1}=P_{k, 0}^{k}$. Note that each $f_{1, j}$ belong to $L^{2}\left(R, \operatorname{ch}^{2} \tau\right)$. This is because $f_{1, j}$ are linear combination of $f_{2}\left(\cdot, \theta_{1}\right)$ and $f_{2}\left(\cdot, \theta_{2}\right)$ for some fixed $\theta_{j}$. Since $Q_{2}(z)$ is not bounded on $(-1,1)[6$, Lemma 8], we can argue as in the proof of Proposition 1 [6] to show that $f_{1,2}$ must vanish. Thus $f=$ $f_{1} P_{k, 0}^{k} e^{-i k \phi}$ for some $f_{1} \in L^{2}\left(R, \operatorname{ch}^{2} \tau\right)$.

Put $W_{k, \mu}^{\ell, \varepsilon}=L^{2}(R)$ or $L^{2}(R)^{2 k+1}\left(k+\varepsilon \in Z_{+}\right)$according as $(\ell, \varepsilon)=(0,0)$ or not, and introduce a Hilbert space $W^{\ell, \varepsilon}$;

$$
W^{\ell, s}=\sum_{k, \mu, k+\varepsilon \in Z_{+}} \oplus W_{k, \mu}^{\ell, \varepsilon} \cdot
$$

Then, in view of Proposition 1.1, an onto isometry $J_{k, \mu}^{\ell, \varepsilon}: \mathscr{W}_{k, \mu}^{\ell, \varepsilon} \rightarrow W_{k, \mu}^{\ell, \varepsilon}$ can be defined by 


$$
J_{k, \mu}^{\ell, s}\left(\sum_{\nu=-k}^{k} f_{\nu} P_{\mu,-\nu}^{k} e^{-i \mu \phi+i(\nu+\varepsilon) \psi}\right)=\left(\sqrt{\frac{2}{2 k+1}} c_{\nu} \operatorname{ch} \tau f_{\nu}\right)
$$

where $c_{\nu}$ is the norm of $e^{i(\nu+\varepsilon) \psi}$ in $\mathfrak{S}_{\pi}$, which is equal to $\Gamma(\ell-\nu+1) / \Gamma(-\ell-\nu)$ or 1 according as $-1<\ell<-1 / 2$ or not. Now we have an onto isometry $J^{\ell, s}=\sum_{k, \mu, k+\varepsilon \in Z_{+}} \oplus J_{k, \mu}^{\ell, \varepsilon}: \mathfrak{S}^{\ell, s} \rightarrow W^{\ell, \varepsilon}$. By the aid of this isometry we shall inquire into actions on $\mathfrak{S}^{\ell, \varepsilon}$ of $\Delta, \Delta^{\prime}, F_{ \pm}$and $F_{3}$. Some calculation similar to that on p. 132 [6] yields

$$
J_{k, k}^{\ell, \varepsilon} \Delta J_{k, k}^{\ell, \varepsilon-1}=L_{k, \ell}, \quad J_{k, k}^{\ell, \varepsilon} \Delta^{\prime} J_{k, k}^{\ell, \varepsilon-1}=M_{k, \ell},
$$

where $L_{k, \ell}$ and $M_{k, \ell}$ are selfadjoint operators taking the following forms.

$$
\begin{aligned}
L_{k, 0}= & -d^{2} / d \tau^{2}+\left\{1 / 4-(k+1 / 2)^{2}\right\} / \operatorname{ch}^{2} \tau, \quad M_{k, 0}=0, \\
L_{k, \ell}= & -d^{2} / d \tau^{2}+A_{k}^{2}\left(1-\operatorname{sh}^{2} \tau\right) / \operatorname{ch}^{2} \tau+i U_{k, \ell} \operatorname{th} \tau / \operatorname{ch} \tau \\
& -\{k(k+1)+\ell(\ell+1)\} / \operatorname{ch}^{2} \tau, \\
M_{k, \ell}= & -2 i A_{k} d / d \tau+V_{k, \ell} / \operatorname{ch} \tau .
\end{aligned}
$$

In the above $A_{k}, U_{k, \ell}$ and $V_{k, \ell}$ are constant matrices in $M_{2 k+1}$. Their $\nu$-th rows $(\nu=k, k-1, \cdots,-k)$ are $\left(\cdots 0_{\nu} 0 \cdots\right),\left(\cdots-a_{\nu} 0 b_{\nu} 0 \cdots\right)$ and $\left(\cdots 0 a_{\nu} 0\right.$ $\left.b_{\nu} 0 \cdots\right)$ respectively, where $b_{\nu}$ is the $(\nu, \nu+1)$-component and

$$
\begin{aligned}
& a_{\nu}=-(\ell+\nu+1) \sqrt{(k-\nu)(k+\nu+1)} \sqrt{|\ell-\nu||| \ell+\nu+1 \mid}, \\
& b_{\nu}=(\ell-\nu+1) \sqrt{(k-\nu+1)(k+\nu)} \sqrt{|\ell+\nu||| l-\nu+1 \mid} .
\end{aligned}
$$

Note that the last factors of $a_{\nu}$ and $b_{\nu}$ are equal to 1 if $\ell=-1 / 2+i n$ $(\eta \geq 0)$. By a theorem [8, p. 287] the domains of $L_{k, 0}, L_{k, \ell}$ and $M_{k, \ell}(\ell \neq 0)$ are $H_{2}(R), H_{2}(R)^{2 k+1}$ and $H_{1}(R)^{2 k+1}$ (or $\left\{\left(f_{\nu}\right) \in L^{2}(R)^{2 k+1} ; f_{\nu} \in H_{1}(R)\right.$ for $\left.\nu \neq 0\right\}$ if $k \in Z_{+}$) respectively. We turn to $F_{ \pm}$and $F_{3}$. At this stage another formulas on $P_{\mu \nu}^{k}$ are required [13, p. 187-188].

$$
\begin{aligned}
\cos \theta P_{\mu \nu}^{k}= & \frac{\sqrt{((k \pm \mu))((k \pm \nu))}}{k(2 k+1)} P_{\mu \nu}^{k-1}+\frac{\mu \nu}{k(k+1)} P_{\mu \nu}^{k} \\
& +\frac{\sqrt{((k \pm \mu+1))((k \pm \nu+1))}}{(k+1)(2 k+1)} P_{\mu \nu}^{k+1}, \\
\cos \frac{\theta}{2} P_{\mu \nu}^{k}= & \frac{1}{2 k+1}\left\{\sqrt{(k-\mu)(k-\nu)} P_{\mu+1 / 2, \nu+1 / 2}^{k-1 / 2}\right. \\
& \left.+\sqrt{(k+\mu+1)(k+\nu+1)} P_{\mu+1 / 2, \nu+1 / 2}^{k+1 / 2}\right\}, \\
\sin \frac{\theta}{2} P_{\mu \nu}^{k}= & \frac{-i}{2 k+1}\left\{-\sqrt{(k-\mu)(k+\nu)} P_{\mu+1 / 2, \nu-1 / 2}^{k-1 / 2}\right. \\
& \left.+\sqrt{(k+\mu+1)(k-\nu+1)} P_{\mu+1 / 2, \nu-1 / 2}^{k-1 / 2}\right\} .
\end{aligned}
$$


The sign of $P_{\mu+1 / 2, \nu-1 / 2}^{k-1 / 2}$ in (1.17) is correct, while the corresponding one on p. 188 [13] is misprinted. Combining (1.16) and (1.17), we obtain

$$
\begin{aligned}
\sin \theta P_{\mu \nu}^{k}= & -2 i\left\{-\frac{\sqrt{((k \pm \mu))((k-\nu-1 / 2 \pm 1 / 2))}}{2 k(2 k+1)} P_{\mu, \nu+1}^{k-1},\right. \\
& -\frac{\mu \sqrt{(k-\nu)(k+\nu+1)}}{k(2 k+2)} P_{\mu, \nu+1}^{k} \\
& \left.+\frac{\sqrt{((k \pm \mu+1))((k+\nu+3 / 2 \pm 1 / 2))}}{(2 k+1)(2 k+2)} P_{\mu, \nu+1}^{k+1}\right\},
\end{aligned}
$$

which is equal to

$$
\begin{aligned}
& -2 i\left\{-\frac{\sqrt{((k \pm \mu))((k+\nu-1 / 2 \pm 1 / 2))}}{2 k(2 k+1)} P_{\mu, \nu-1}^{k-1}\right. \\
& +\frac{\mu \sqrt{(k-\nu+1)(k+\nu)}}{k(2 k+2)} P_{\mu, \nu-1}^{k} \\
& \left.+\frac{\sqrt{((k \pm \mu+1))((k-\nu+3 / 2 \pm 1 / 2))}}{(2 k+1)(2 k+2)} P_{\mu, \nu-1}^{k+1}\right\},
\end{aligned}
$$

for $P_{\mu \nu}^{k}=P_{-\mu,-\nu}^{k}\left[13\right.$, p. 123]. Now assume $(\ell, \varepsilon) \neq(0,0)$ and let $f_{\nu} \in C_{0}^{\infty}(R)$ $(\nu=k, k-1, \cdots,-k)$. Then, making use of (1.15), (1.18) and a formula [13, p. 137]

$$
\begin{aligned}
d / d \theta P_{\mu \nu}^{k}(\cos \theta)= & i\left(\sqrt{(k-\nu)(k+\nu+1)} P_{\mu, \nu+1}^{k}(\cos \theta)\right. \\
& \left.+\sqrt{(k-\nu+1)(k+\nu)} P_{\mu, \nu-1}^{k}(\cos \theta)\right) / 2,
\end{aligned}
$$

we can show

$$
\begin{aligned}
F_{3} \sum_{\nu=-k}^{k} & f_{\nu} P_{\mu,-\nu}^{k} e^{-i \mu \phi+i(\nu+\varepsilon) \psi} \\
= & \frac{\sqrt{(k-\mu)(k+\mu)}}{2 k(2 k+1)} \sum_{\nu=-k}^{k}\left[2 i \sqrt{(k-\nu)(k+\nu)}\left\{f_{\nu}^{\prime}+(k+1) \operatorname{th} \tau f_{\nu}\right\}\right. \\
& +\left\{-(\ell+\nu+1) \sqrt{(k+\nu)(k+\nu+1)} f_{\nu+1}\right. \\
& \left.\left.-(\ell-\nu+1) \sqrt{(k-\nu)(k-\nu+1)} f_{\nu-1}\right\} / \operatorname{ch} \tau\right] \\
& \times P_{\mu,-\nu}^{k-1} e^{-i \mu \phi+i(\nu+\varepsilon) \psi}+\frac{\mu}{2 k(k+1)} \sum_{\nu=-k}^{k}\left[-2 i\left(f_{\nu}^{\prime}+\operatorname{th} \tau f_{\nu}\right)\right. \\
& +\left\{-(\ell+\nu+1) \sqrt{(k-\nu)(k+\nu+1)} f_{\nu+1}\right. \\
& \left.\left.+(\ell-\nu+1) \sqrt{(k-\nu+1)(k+\nu)} f_{\nu-1}\right\} / \operatorname{ch} \tau\right] P_{\mu,-\nu}^{k} e^{-i \mu \phi+i(\nu+\varepsilon) \psi} \\
& +\frac{\sqrt{(k-\mu+1)(k+\mu+1)}}{2(k+1)(2 k+1)} \sum_{\nu=-k}^{k}[2 i \sqrt{(k-\nu+1)(k+\nu+1)} \\
& \times\left(f_{\nu}^{\prime}-k \operatorname{th} \tau f_{\nu}\right)+\left\{(\ell+\nu+1) \sqrt{(k-\nu)(k-\nu+1)} f_{\nu+1}\right. \\
& \left.\left.+(\ell-\nu+1) \sqrt{(k+\nu)(k+\nu+1)} f_{\nu-1}\right\} / \operatorname{ch} \tau\right] P_{\mu,-\nu}^{k+1} e^{-i \mu \phi+i(\nu+\varepsilon) \psi} .
\end{aligned}
$$


Thanks to (1.19), (1.18) and a formula [13, p. 136]

$$
\left\{\sqrt{1-z^{2}} d / d z+\frac{\mu z-\nu}{1-z^{2}}\right\} P_{\mu \nu}^{k}(z)=-i \sqrt{(k-\mu)(k+\mu+1)} P_{\mu+1, \nu}^{k}(z),
$$

we can spare much calculation in reducing $F_{ \pm} \sum_{\nu=-k}^{k} f_{\nu} P_{\mu,-\nu}^{k} e^{-i \mu \dot{\phi}+i(\nu+\varepsilon) \psi}$, for $F_{-}=\left[H_{-}, F_{3}\right]$ and $F_{+}=\left[F_{3}, H_{+}\right]$as they ought to be [12, p. 77]. The case $(\ell, \varepsilon)=(0,0)$ needs no separate consideration. The results in this case are obtainable from those in the case $(\ell, 0)$ by setting $\ell=0$ and $f_{\nu}=0$ for $\nu \neq 0$. To sum up,

LEMMA 1.2. Let $f=\left(f_{k^{\prime}, \mu^{\prime}}\right)$ be an element of $W^{\ell, s}$ whose $(k, \mu)$-component $f_{k, \mu}$ alone does not necessarily vanish and lies in $H_{1}(R)$ or $H_{1}(R)^{2 k+1}$ according as $(\ell, \varepsilon)=(0,0)$ or not. Then $f$ belongs to the domains of $J^{\ell, \varepsilon} F_{s} J^{\ell, \varepsilon-1}(s= \pm$, 3). Moreover, omitting the suffix $(\ell, \varepsilon)$ of $J^{\ell, \varepsilon}$, we have the following relations.

$$
\begin{aligned}
& \left(J F_{3} J^{-1} f\right)_{k-1, \mu}=\frac{\sqrt{(k-\mu)(k+\mu)}}{2 k \sqrt{(2 k-1)(2 k+1)}} K_{+, k-1, \ell}^{*} f_{k, \mu}, \\
& \left(J F_{3} J^{-1} f\right)_{k, \mu}=\frac{\mu}{2 k(k+1)} M_{k, \ell} f_{k, \mu}, \\
& \left(J F_{3} J^{-1} f\right)_{k+1, \mu}=\frac{\sqrt{(k-\mu+1)(k+\mu+1)}}{2(k+1) \sqrt{(2 k+1)(2 k+3)}} K_{+, k, \ell} f_{k, \mu}, \\
& \left(J F_{3} J^{-1} f\right)_{k^{\prime}, \mu^{\prime}}=0 \quad \text { otherwise. } \\
& \left(J F_{ \pm} J^{-1} f\right)_{k-1, \mu \pm 1} \propto K_{+, k-1, \ell}^{*} f_{k, \mu}, \quad\left(J F_{ \pm} J^{-1} f\right)_{k, \mu \pm 1} \propto M_{k, \ell} f_{k, \mu}, \\
& \left(J F_{ \pm} J^{-1} f\right)_{k+1, \mu \pm 1} \propto K_{+, k, \ell} f_{k, \mu}, \quad\left(J F_{ \pm} J^{-1} f\right)_{k^{\prime}, \mu^{\prime}}=0 \quad \text { otherwise. }
\end{aligned}
$$

In the above $K_{+, k, 0}=2 i(k+1)\{d / d \tau-(k+1)$ th $\tau\}\left(k \in Z_{+}\right)$, and in the case $\ell \neq 0$,

$$
K_{+, k, \ell}=2 i B_{k}\{d / d \tau-(k+1) \text { th } \tau\}+Y_{k, \ell} / \operatorname{ch} \tau,
$$

where $B_{k}$ and $Y_{k, \ell}$ are constant matrices in $M_{2 k+3,2 k+1}$. Their $\nu$-th rows $(\nu=k+1, k, \cdots,-k-1)$ are $(\cdots 0 \sqrt{(k-\nu+1)(k+\nu+1)} 0 \cdots)$ and $(\cdots 0(\ell+\nu+1) \sqrt{(k-\nu)(k-\nu+1)} \sqrt{|\ell-\nu||| \ell+\nu+1 \mid} 0(\ell-\nu+1) \times$ $\sqrt{(k+\nu)(k+\nu+1)} \sqrt{|\ell+\nu||| \ell-\nu+1 \mid} 0 \cdots)$ respectively, in particular their $(\nu, \nu)$-components are equal to $\sqrt{(k-\nu+1)(k+\nu+1)}$ and 0 respectively.

Proof. For the sake of definiteness assume $(\ell, \varepsilon) \neq(0,0)$. When $f_{k, \mu}$ lies in $C_{0}^{\infty}(R)^{2 k+1},(1.21)$ and (1.22) hold. To conclude the proof, it is enough to recall that $C_{0}^{\infty}(R)^{2 k+1}$ is dense in $H_{1}(R)^{2 k+1}$ and that the infinitesimal operators $\omega_{j}(4 \leq j \leq 6)$ are closed operators.

Q.E.D. 
We shall now show that, if there exists a $P_{+}$-invariant, closed proper subspace $\mathscr{D}$ of $\mathscr{S}^{\ell, \varepsilon}$, there is a nontrivial sequence $\left\{D_{k}\right\}_{k \in Z_{++}}$satisfying certain conditions. For this purpose, set

$$
T_{t}=J^{\ell, \varsigma} U^{\ell, \varepsilon}(t / M, 0,0,0, e) J^{\ell, \varepsilon-1}=\exp \{i t \operatorname{sh} \tau\} .
$$

Lemma 1.3. Suppose there exists a $P_{+}$-invariant, closed proper subspace $\mathscr{D}$ of $\mathfrak{S}^{\ell, \varepsilon}$, and put $\mathscr{D}_{k, \mu}=\mathscr{D} \cap \mathscr{W}_{k, \mu}^{\ell, \varepsilon}\left(k \in Z_{+}+\varepsilon\right)$. Then,

(i) $\mathscr{D}_{k, k}$ is a proper closed subspace of $\mathscr{W}_{k, k}^{\ell, \varepsilon}$ and invariant under selfadjoint operators $\Delta, \Delta^{\prime}$ and the semigroup $U^{\ell, s}(t, 0,0,0, e)(t \geq 0)$,

(ii) $F_{+} \mathscr{D}_{k, k} \subset \mathscr{D}_{k+1, k+1}$ and $F_{-} \mathscr{D}_{k, k} \subset \sum_{j=-1}^{1} \oplus \mathscr{D}_{k+j, k-1}$.

Proof. The statement (ii) holds because of (34) and (35) [12, p. 106]. Another way to prove (ii) directly is to use Lemma 1.2 and the fact that $\mathfrak{S}^{\ell, s}$ $=\sum_{k, \mu} \oplus \mathscr{W}_{k, \mu}^{\ell, \varepsilon}$. Since $H_{3}$ and $\Delta_{0}$ commute with $\Delta, \Delta^{\prime}$ and $U^{\ell, \varepsilon}(t, 0,0,0, e)$, (i) follows except that $\mathscr{D}_{k, k}$ is a proper subspace. Assuming that $\mathscr{D}_{k, k}=\{0\}$ for some $k \in Z_{+}+\varepsilon$, we shall show that $\mathscr{D}_{k, k}=\{0\}$ for any $k$. First, $\mathscr{D}_{k^{\prime}, k^{\prime}}$ $=\{0\}$ for $k^{\prime} \leq k$, because, if $\mathscr{D}_{k^{\prime}, k^{\prime}} \neq\{0\}$ then $F_{+} \mathscr{D}_{k^{\prime}, k^{\prime}} \neq\{0\}$ (see Lemma 2 (iii) [6]). Secondly, $\mathscr{D}_{k+1, k+1}=\{0\}$. To prove this, set $G_{\alpha}=(\alpha-i \operatorname{sh} \tau)^{-1}$ $(\operatorname{Re} \alpha>0)$, which we regard as the resolvent of the semigroup $U^{\ell, \varepsilon}(t / M, 0$, $0,0, e)$ or $T_{t}(t \geq 0)$. Since $\mathscr{W}_{k, k}^{\ell, \epsilon} \subset \mathscr{D}^{\perp}$ and since $\mathscr{D}^{\perp}$ is invariant under $U^{\ell, \varepsilon}(0, g)(g \in G)$ and $U^{\ell, \varepsilon}(-t, 0,0,0, e)(t>0)$, there results, for any $f \in J^{\ell, \varepsilon}$ $\mathscr{D}_{k+1, k+1}$ and $h \in C_{0}^{\infty}(R)\left(\subset W_{k, k}^{\ell, \varepsilon}\right)$,

$$
\left\langle f, J_{k+1, k+1}^{\ell, \varepsilon} F_{+} J_{k, k}^{\ell, \varepsilon-1} h\right\rangle=0, \quad\left\langle G_{\alpha} f, J_{k+1, k+1}^{\ell, \varepsilon} F_{+} J_{k, k}^{\ell, \varepsilon-1} h\right\rangle=0 .
$$

From now on let $(\ell, \varepsilon) \neq(0,0)$. Another case is easier to handle. We recall that a locally integrable function on $R$ having a locally integrable derivative in the distribution sense is absolutely continuous. Set $f=\left(f_{\nu}\right)$. Then the first equality in (1.25) implies that $f_{\nu}(|\nu| \leq k)$ is absolutely continuous, and the second one now can be rewritten as

$$
2 i\left\langle B_{k} G_{\alpha}^{\prime} f, h\right\rangle+\left\langle f, K_{+, k, \ell} G_{\alpha}^{*} h\right\rangle=0 .
$$

$\mathscr{W}_{k, k}^{\ell, \varepsilon}$ being invariant under $U^{\ell, \varepsilon}(--t, 0,0,0, e)(t \geq 0)$, the second term vanishes. Now (1.26) yields $f_{\nu}=0(|\nu| \leq k)$, and the first equality in (1.25) implies $f=0$, as desired. Similarly it can be shown that if $\mathscr{D}_{k, k}=\mathscr{V}_{k, k}^{\ell, e}$ for some $k$ the same is true for any $k$.

Q.E.D.

We shall give a more manageable necessary condition for $\left(U^{\ell, \varepsilon}, \mathfrak{S}_{c}^{\ell, \varepsilon}\right)$ to have a $P_{+}$-invariant, closed proper subspace.

Lemma 1.4. Suppose there exists a $P_{+}$-invariant, closed proper subspace 
in $\mathfrak{S}^{\ell, \varepsilon}$. Then there is a sequence $\left\{D_{k} ; k \in Z_{+}+\varepsilon, D_{k}\right.$ is a proper closed subspace of $L^{2}(R)^{2 k+1}$ (or $L^{2}(R)$ if $\left.\left.(\ell, \varepsilon)=(0,0)\right)\right\}$ which satisfies (Q.1) and (Q.2);

(Q.1) $D_{k}$ is a closed subspace of $L^{2}(R)^{2 k+1}$ (or $\left.L^{2}(R)\right)$ and invariant under the selfadjoint operators $L_{k, \ell}, M_{k, \ell}$ and the semigroup $T_{t}(t \geq 0)$.

(Q.2) $K_{+, k, \ell} D_{k} \subset D_{k+1}$ and $K_{+, k-1, \ell}^{*} D_{k} \subset D_{k-1}$, where the domains of the operators are $H_{2}(R)^{2 k+1}\left(\right.$ or $H_{2}(R)$ if $(\ell, \varepsilon)=(0,0)$ ).

Proof. We retain the notation in Lemma 1.3. Put $D_{k}=J_{k, k}^{\ell, \varepsilon} \mathscr{D}_{k, k}$. We shall show that the sequence $\left\{D_{k}\right\}_{k \in Z_{++}}$satisfies (Q.1) and (Q.2). By (1.11) and (1.24) Lemma 1.3 (i) implies (Q.1). Denote by $E_{k, \mu}^{\ell, \varepsilon}$ the orthogonal projection: $\mathfrak{S}^{\ell, \varepsilon} \rightarrow \mathscr{W}_{k, \mu}^{\ell, s}, J_{k+1, k+1}^{\ell, \varepsilon} F_{+} J_{k, k}^{\ell, s-1} \propto K_{+, k, \ell}$ and $J_{k-1, k-1}^{\ell, \varepsilon} E_{k-1, k-1}^{\ell, \varepsilon} F_{-} J_{k, k}^{\ell, s-1}$ $\propto K_{+, k-1, \ell}^{*}$ on account of Lemma 1.2. Now Lemma 1.3 (ii) implies (Q.2).

Q.E.D.

In case $(\ell, \varepsilon)=(0,0)$, all sequences $\left\{D_{k} ; k \in Z_{+}, D_{k}\right.$ is a closed proper subspace of $L^{2}(R)$ \} satisfying (Q.1) and (Q.2) can be determined as a result of the Part II [7, $\S 1]$. Indeed, using the notation there, it is clear that $L_{k, 0}=\mathscr{L}_{k+1 / 2,0}, K_{+, k, 0} \propto F_{+, k+1 / 2,0}$ and $K_{+, k-1,0}^{*} \propto F_{-, k+1 / 2,0}$. Therefore we get

Proposition 1.5. Let $D_{k}$ be a closed proper subspace of $L^{2}(R)\left(k \in Z_{+}\right)$. Then $\left\{D_{k}\right\}_{k \in Z_{+}}$satisfies (Q.1) and (Q.2) iff it coincides with either $\left\{D_{k+1 / 2,-}^{0}\right\}_{k \in Z_{+}}$ or $\left\{D_{k+1 / 2,+}^{0}\right\}_{k \in Z_{+}}$.

Proof. The sequences $\left\{D_{k+1 / 2, \pm}^{0}\right\}$ satisfy the conditions (Q.1) and (Q.2) by Theorem 1.4 [7]. In view of Theorem 1.2 [7], $D_{0}$ coincides with one of $D_{1 / 2, \pm}^{0}$. Theorem 1.3 and the relation (1.32) [7] now imply that $D_{k}=D_{k+1 / 2, \pm}^{0}$ according as $D_{0}=D_{1 / 2, \pm}^{0}$.

Q.E.D.

In case $(\ell, \varepsilon) \neq(0,0)$, an analogue of Proposition 1.5 will be obtained later (Theorems 2.2 and 3.1). Throughout the rest of this section we shall be exclusively concerned with the case $(\ell, \varepsilon)=(0,0)$. Correspondingly $k$ runs in $Z_{+}$. Define subspaces $\mathscr{D}_{ \pm}^{0,0}$ of $\mathfrak{S}^{0,0}$ by

$$
\mathscr{D}_{ \pm}^{0,0}=\sum_{k, \mu, k \in Z_{+}} \oplus J_{k, \mu}^{0,0-1} D_{k+1 / 2, \pm}^{0} \text {. }
$$

Now we are ready to state one of our main theorems in this paper.

THEOREM 1.1. Let $\mathscr{D}$ be a closed proper subspace of $\mathfrak{S}^{0,0}$. Then $\mathscr{D}$ is $P_{+}$-invariant iff it coincides with one of $\mathscr{D}_{ \pm}^{0,0}$.

TheOREM 1.2. The representations of $S L(2, C)$ realized in $\mathscr{D}_{ \pm}^{0,0}$ decompose into irreducible ones as 


$$
\begin{array}{ll}
\int_{R_{+}}^{\oplus} \mathfrak{S}_{0, \rho} d \rho \oplus \sum_{n-1 \in Z_{+}} \oplus \mathfrak{S}_{2 n, 0} & \text { in } \mathscr{D}_{+}^{0,0}, \\
\int_{R_{+}}^{\oplus} \mathfrak{S}_{0, \rho} d \rho & \text { in } \mathscr{D}_{-}^{0,0} .
\end{array}
$$

Remark. Mukunda [11] has shown that the representation $\left(U^{0,0}, \mathfrak{S}^{0,0}\right)$ of $S L(2, C)$ decomposes into irreducible ones as

$$
\left([2] \int_{R_{+}}^{\oplus} \widetilde{S}_{0, \rho} d \rho\right) \oplus \sum_{n-1 \in Z_{+}} \oplus \widetilde{S}_{2 n, 0} .
$$

Proof of Theorem 1.1. 1) We shall show that the condition is necessary. To this end set $\mathscr{D}_{k, \mu}=\mathscr{D} \cap \mathscr{W}_{k, \mu}^{0,0}$. Then $\mathscr{D}_{k, k}=J_{k, k}^{0,0-1} D_{k+1 / 2, \pm}^{0}$ by Lemmas 1.3, 1.4 and Proposition 1.5. Since $H_{-}^{k-\mu} \mathscr{D}_{k, k}=\mathscr{D}_{k, \mu}, \mathscr{D}_{k, \mu}=J_{k, \mu}^{0,0-1}$ $D_{k+1 / 2, \pm}^{0}$ on account of (1.6) and (1.8). Consequently $\mathscr{D}=\sum_{k, \mu} \oplus \mathscr{D}_{k, \mu}=\mathscr{D}_{ \pm}^{0,0}$. 2) We shall show that $\mathscr{D}_{ \pm}^{0,0}$ are $P_{+}$-invariant. It is evident that $J_{k, \mu}^{0,0-1}$ $D_{k+1 / 2}^{0}$, are invariant for $U^{0,0}(t, 0,0,0, e)(t \geq 0)$. Since $H_{-}$and $H_{3}$ leave $\mathscr{D}_{k, \pm}=\sum_{\mu=-k}^{k} \oplus J_{k, \mu}^{0,0-1} D_{k+1 / 2, \pm}^{0}$ invariant, $\mathscr{D}_{k, \pm}$ are $S U(2)$-invariant. It suffices to verify that $\mathscr{D}_{ \pm}^{0,0}$ is invariant under $U^{0,0}\left(0, \omega_{6}(t)\right)(t \in R)$, for the semigroup $(t, 0,0,0, e)(t \geqslant 0)$ and $G$ generate $P_{+}$topologically, $G$ being generated by $S U(2)$ and the one-parameter subgroup $\omega_{6}(t)$. For this purpose put $D_{k, \mu, \pm}$ $=D_{k+1 / 2, \pm}^{0}, \tilde{D}_{k, \mu, \pm}=\tilde{D}_{k+1 / 2, \pm}^{0}$ and $\hat{D}_{k, \mu, \pm}=\hat{D}_{k+1 / 2, \pm}^{0}$, then we have onto isometries $\mathscr{F}_{k+1 / 2}: D_{k, \mu, \pm} \rightarrow \tilde{D}_{k, \mu, \pm}$ and $I_{ \pm, k+1 / 2}: \tilde{D}_{k, \mu, \pm} \rightarrow \hat{D}_{k, \mu, \pm}$ (see (1.34) [7]). Regarding $D_{k, \mu, \pm}$ as subspaces of $W_{k, \mu}^{0,0}$ (see (1.10)), define subspaces $D_{ \pm}=$ $\sum_{k, \mu} \oplus D_{k, \mu, \pm} \quad$ of $\quad W^{0,0}$. Set $\tilde{D}_{ \pm}=\sum_{k, \mu} \oplus \tilde{D}_{k, \mu, \pm} \quad$ and $\hat{D}_{ \pm}=\sum_{k, \mu} \oplus \hat{D}_{k, \mu, \pm}$. Then we can naturally define onto isometries $\mathscr{F}_{ \pm}: D_{ \pm} \rightarrow \tilde{D}_{ \pm}$and $I_{ \pm}: \tilde{D}_{ \pm} \rightarrow$ $\hat{D}_{ \pm}$in terms of $\mathscr{F}_{k+1 / 2}$ and $I_{ \pm, k+1 / 2}$ respectively. It clear that $D_{ \pm}=J^{0,0} \mathscr{D}_{ \pm}^{0,0}$. Let us further define dense subspaces $\hat{D}_{ \pm, c}$ of $\hat{D}_{ \pm}$. Put $\hat{D}_{k, \mu, \pm, c}=C_{0}\left(R_{+}\right)^{1}$ $\oplus E_{k+1 / 2, \pm}$ and denote by $\hat{D}_{ \pm, c}$ the algebraic sum $\sum_{k, \mu} \oplus \hat{D}_{k, \mu, \pm, c}$. By Lemma 2.4 [7] it is enough to show that $F_{3}$ restricted to $\mathscr{D}_{ \pm, c}^{0,0}=\left(I_{ \pm} \mathscr{F}_{ \pm} J^{0,0}\right)^{-1} \hat{D}_{ \pm, c}$ is essentially selfadjoint in $\mathscr{D}_{ \pm}^{0,0}$. To this end, set

$$
i \hat{\omega}_{6, \pm}=\left(I_{ \pm} \mathscr{F}_{ \pm} J^{0,0}\right) F_{3}\left(I_{ \pm} \mathscr{F}_{ \pm} J^{0,0}\right)^{-1} \text { with domain } \hat{D}_{ \pm, c},
$$

and let us prove that $i \hat{\omega}_{6, \pm}$ is essentially selfadjoint in $\hat{D}_{ \pm}$. Let $\hat{h}=\left(\hat{h}_{k^{\prime}, \mu^{\prime}}\right)$ be an element of $\hat{D}_{ \pm, c}$ with $\hat{h}_{k^{\prime}, \mu^{\prime}}=0$ for $\left(k^{\prime}, \mu^{\prime}\right) \neq(k, \mu)$. Then, by (1.35) and (1.33) [7] we obtain

$$
\begin{aligned}
\left(i \hat{\omega}_{6} \hat{h}\right)_{k \pm 1, \mu}(\lambda)= & \pm i \sqrt{\frac{(k-\mu+1 / 2 \pm 1 / 2)(k+\mu+1 / 2 \pm 1 / 2)}{(2 k \pm 1)(2 k+2 \pm 1)}} \\
& \times \sqrt{(k+1 / 2 \pm 1 / 2)^{2}+\lambda} \hat{h}_{k, \mu},(\lambda)(\lambda>0)
\end{aligned}
$$




$$
\begin{aligned}
& \left(i \hat{\omega}_{6,+} \hat{h}\right)_{k \pm 1, \mu}\left(-(j-1 / 2)^{2}\right)=\mp i \sqrt{\frac{(k-\mu+1 / 2 \pm 1 / 2)(k+\mu+1 / 2 \pm 1 / 2)}{(2 k \pm 1)(2 k+2 \pm 1)}} \\
& \quad \times \sqrt{(k+1 / 2 \pm 1 / 2)^{2}-(j-1 / 2)^{2}} \hat{h}_{k, \mu}\left(-(j-1 / 2)^{2}\right), \\
& \left(i \hat{\omega}_{6} \hat{h}\right)_{k^{\prime}, \mu^{\prime}}(\lambda)=0 \quad \text { for }\left(k^{\prime}, \mu^{\prime}\right) \neq(k \pm 1, \mu) .
\end{aligned}
$$

In the above $\hat{\omega}_{6}$ stands for one of $\hat{\omega}_{6, \pm}$. Applying Proposition 1.6 in the case $(m, \rho)=(2 j-1,0)$ to $i \hat{\omega}_{6,+}$ in $\left\{\hat{h} \in \hat{D}_{+} ; \hat{h}_{k, \mu}(\lambda)=0\right.$ except for $\lambda=$ $\left.-(j-1 / 2)^{2}\right\}, i \hat{\omega}_{6,+}$ turns out to be essentially selfadjoint there $\left(j \in Z_{+}+3 / 2\right)$. Put $\hat{D}_{+}^{+}=\left\{\hat{h} \in \hat{D}_{+} ; \hat{h}_{k, \mu}(\lambda)=0\right.$ for $\left.\lambda<0\right\}$. We shall prove that $\hat{\omega}_{b_{+}+}$, which is symmetric, is essentially selfadjoint in $\hat{D}_{+}^{+}$too by showing that the image $\left(i \hat{\omega}_{6,+}-z\right)\left(\hat{D}_{+}^{+} \cap \hat{D}_{+, c}\right)$ is dense in $\hat{D}_{+}^{+}$for any $z(\operatorname{Im} z \neq 0)$. If an $\hat{f} \in \hat{D}_{+}^{+}$is orthogonal to the image, it follows from (1.28) that

$$
\begin{aligned}
& i \sqrt{\frac{((k \pm \mu))}{((2 k \pm 1))} \sqrt{k^{2}+\lambda} \hat{f}_{k-1, \mu}(\lambda)-z^{*} \hat{f}_{k, \mu}(\lambda)} \\
& \quad-i \sqrt{\frac{((k \pm \mu+1))}{((2 k+2 \pm 1))}} \sqrt{(k+1)^{2}+\lambda} \hat{f}_{k+1, \mu}(\lambda)=0 \text { a.e. }
\end{aligned}
$$

On the other hand, using the notation in Proposition 1.6, let $U$ be a unitary operator on $\ell_{0,2}^{2} \sqrt{\lambda}$ such that $U f_{\mu}^{k}=(-1)^{k} f_{\mu}^{k}$. Evidently $U \dot{F}_{3} U^{-1}$ is also essentially selfadjoint and satisfies

$$
U \dot{F}_{3} U^{-1} f_{\mu}^{k}=-\sqrt{((k \pm \mu))} c_{k} f_{\mu}^{k-1}-a_{k} f_{\mu}^{k}+\sqrt{((k \pm \mu+1))} c_{k+1} f_{\mu}^{k+1} .
$$

Now (1.30) yields $f_{k, \mu}(\lambda)=0$ a.e. on $R_{+}$, namely $f=0$. The proof of essentially selfadjointness of $i \hat{\omega}_{6,-}$ is similar.

Q.E.D.

Proof of Theorem 1.2. Put $\mathscr{D}_{k, \mu, \pm}=\mathscr{D}_{ \pm}^{0,0} \cap \mathscr{W}_{k, \mu}^{0,0}$. For the proof it is enough to determine the spectral type of selfadjoint operators $\Delta \mid \mathscr{D}_{0,0, \pm}$ and $\Delta^{\prime} \mid \mathscr{D}_{k, k, \pm} \ominus F_{+} \mathscr{D}_{k-1, k-1, \pm}\left(k \in Z_{+}+1\right)[6, \S 3]$. The following unitary equivalence relations are clear.

$$
\Delta\left|\mathscr{D}_{0,0, \pm} \simeq L_{0,0}\right| D_{1 / 2, \pm}^{0}=\mathscr{L}_{1 / 2,0} \mid D_{1 / 2, \pm}^{0} \simeq \int_{R_{+}}^{\oplus} \lambda d \lambda .
$$

On the other hand, by (1.32) [7] and (1.22) it can be easily seen that $\mathscr{D}_{k, k,-} \ominus F_{+} \mathscr{D}_{k-1, k-1,-}=\{0\}$ and $\mathscr{D}_{k, k,+} \ominus F_{+} \mathscr{D}_{k-1, k-1,+} \subset\left\{\int_{k, k}^{0,0-1} e_{k+1 / 2, k+1 / 2}\right\} . \quad$ As to the definition of $e_{k+1 / 2, k+1 / 2}$, see Lemma 1.8 [7]. We claim that the opposite inclusion relation also holds. To prove this, suppose an $f \in$ $\mathscr{D}_{k-1, k-1,+}$ lies in the domain of $F_{+}$. Then $\left\langle J_{k, k}^{0,0-1} e_{k+1 / 2, k+1 / 2}, F_{+} f\right\rangle=\left\langle F_{-} J_{k, k}^{0,0-1}\right.$ $\left.e_{k+1 / 2, k+1 / 2}, f\right\rangle=\left\langle 0, J_{k-1, k-1}^{0,0} f\right\rangle=0$, for $K_{+, k-1}^{*} e_{k+1 / 2, k+1 / 2}=0$. Since $\Delta^{\prime}=0$, we have 


$$
\Delta^{\prime} \mid \mathscr{D}_{k, k,+} \ominus F_{+} \mathscr{D}_{k-1, k-,+} \simeq \int_{R}^{\oplus} \lambda d \delta(\lambda)
$$

where $\delta$ is the Dirac measure with unit mass at $\lambda=0$.

The proof of Theorem 1.1 relies on the next proposition, which asserts that the operators $i \omega_{j}(1 \leq j \leq 6)$ for the irreducible unitary representation $\widetilde{S}_{m, \rho}[12, \S 11]$ are essentially selfadjoint even if they are restricted to the algebraic linear span of the canonical basis.

Proposition 1.6. Let $\ell_{m, \rho}^{2}$ be a Hilbert space $\left\{\left(a_{k, \mu}\right) ; k=m / 2, m / 2+1\right.$, $\cdots, \mu=-k,-k+1, \cdots, k$ with $\left.\sum_{k, \mu}\left|a_{k, \mu}\right|^{2}<\infty\right\}$ for $(m, \rho) \in\{(0, \rho) ; \rho \geq 0\}$ $\cup\left(Z_{+}+1\right) \times R$, and denote by $\ell_{m, \rho, c}^{2}$ a dense subspace $\left\{\left(a_{k, \mu}\right) \in \ell_{m, \rho}^{2} ; a_{k, \mu}=0\right.$ for large $k\}$. Then following operators $i \dot{\omega}_{j, m, \rho}$ in $\ell_{m, \rho}^{2}$ with domain $\ell_{m, \rho, c}^{2}$ are essentially selfadjoint. In order to define these operators, let $f_{\mu}^{k}$ be an element of $\ell_{m, \rho}^{2}$ with $\left(k^{\prime}, \mu^{\prime}\right)$-component $\delta_{k k^{\prime}} \delta_{\mu \mu^{\prime}}$ and put

$$
\dot{H}_{ \pm}=i \dot{\omega}_{2} \pm \dot{\omega}_{1}, \quad \dot{H}_{3}=i \dot{\omega}_{3}, \quad \dot{F}_{ \pm}=i \dot{\omega}_{5} \pm \dot{\omega}_{4}, \quad F_{3}=i \dot{\omega}_{6},
$$

where the suffix $(m, \rho)$ is omitted for the brevity. Then we define $i \dot{\omega}_{j, m, \rho}$ indirectly by requiring the following equalities.

$$
\begin{aligned}
\dot{H}_{ \pm} f_{\mu}^{k}= & \sqrt{(k \pm \mu+1)(k \mp \mu)} f_{\mu \pm 1}^{k}, \quad \dot{H}_{3} f_{\mu}^{k}=\mu f_{\mu}^{k}, \\
\dot{F}_{ \pm} f_{\mu}^{k}= & \pm \sqrt{(k \mp \mu)(k \mp \mu-1)} c_{k} f_{\mu \pm 1}^{k-1}-\sqrt{(k \mp \mu)(k \pm \mu+1)} a_{k} f_{\mu \pm 1}^{k} \\
& \pm \sqrt{(k \pm \mu+1)(k \pm \mu+2)} c_{k+1} f_{\mu \pm 1}^{k+1}, \\
\dot{F}_{ \pm} f_{\mu}^{k}= & \sqrt{((k \pm \mu))} c_{k} f_{\mu}^{k-1}-\mu a_{k} f_{\mu}^{k}-\sqrt{((k \pm \mu+1))} c_{k+1} f_{\mu}^{k+1}
\end{aligned}
$$

where $\left.a_{k}=m \rho /\{4 k(k+1)\}, c_{k}=i \sqrt{\left(k^{2}-m^{2} / 4\right)\left(k^{2}+\rho^{2} / 4\right) /\left\{k^{2}\left(4 k^{2}-1\right)\right.}\right][12, \mathrm{p}$. 110 and p. 152].

Remark. Quite analogous statement holds for an irreducible unitary representation belonging to the supplementary series of $S L(2, C)$, as one can infer from our proof of the proposition.

CoRollary. Let $\stackrel{\circ}{F}_{3}$ be an operator in $\ell_{m, \rho}^{2}$ with domain $\ell_{m, \rho, c}^{2}$ such that

$$
{\stackrel{\circ}{F_{3}}}_{3} f_{\mu}^{k}=-i \sqrt{((k \pm \mu))} c_{k} f_{\mu}^{k-1}+\mu a_{k} f_{\mu}^{k}-i \sqrt{((k \pm \mu+1))} c_{k+1} f_{\mu}^{k+1} .
$$

Then $\stackrel{\circ}{F}_{3}$ is essentially selfadjoint.

Proof of the corollary. Indeed, $\stackrel{\circ}{F}_{3}$ is unitarily equivalent to $\dot{F}_{3}$ under a unitary operator $U$ sending $f_{\mu}^{k}$ to $(-i)^{k} f_{-\mu}^{k}$.

Q.E.D.

To shorten the proof of Proposition 1.6, we prepare two lemmas. 
LEMMA 1.7. Let $R$ be the right regular representation of $S U(2)$, and denote by $R_{i}(1 \leq i \leq 3)$ and $\Delta_{R}$ the infinitesimal operator $d / d t R\left(\omega_{i}(t)\right)_{t=0}$ and the Laplace operator $\sum_{i=1}^{3} R_{i}^{2}$ respectively. For an $f \in C^{\infty}(S U(2))$ define $f_{n}\left(n \in Z_{+} / 2\right)$ by

$$
f_{n}=\sum_{k=0}^{n} \sum_{\mu, \nu=-k}^{k}(2 k+1)\left\langle c_{\mu \nu}^{k}, f\right\rangle c_{\mu \nu}^{k},
$$

where $c_{\mu \nu}^{k}$ is the matrix element of the representation $\widetilde{S}_{2 k}$ of $S U(2)[12, \mathrm{p}$. $58]$ and $\langle$,$\rangle denotes the inner product on L^{2}(S U(2))$ relative to the normalized Haar measure. Then $\left\|\left(1-\Delta_{R}\right)^{q}\left(f-f_{n}\right)\right\|$ and $\left\|\left(1-\Delta_{R}\right)^{q} R_{i}\left(f-f_{n}\right)\right\|\left(q \in Z_{+}\right)$ tend to zero as $n \rightarrow \infty$. In particular $f_{n}$ (resp. $R_{i} f_{n}$ ) converge to $f$ (resp. $\left.R_{i} f\right)$ as $n \rightarrow \infty$ relative to the uniform norm.

Proof. The $L^{2}$-norms tend to zero on account of the Peter-Weyl theorem and the fact that $\Delta_{R} c_{\mu \nu}^{k}=-k(k+1) c_{\mu \nu}^{k}$. The Sobolev lemma [9, p. 51] now implies the convergence with respect to the uniform norm.

Q.E.D.

After Neumark [12, p. 143] we denote by $\left(V, L_{m}^{2}(S U(2))\right)$ the irreducible unitary representation $\mathfrak{S}_{m, \rho}$ realized in $L_{m}^{2}(S U(2))$.

Lемма 1.8. Let $\omega_{j}(1 \leq j \leq 6)$ be the infinitesimal operator for the representation $\left(V, L_{m}^{2}(S U(2))\right)$. Then there exist smooth functions $a_{j i}(0 \leq$ $i \leq 3)$ on $S U(2)$ such that

$$
\omega_{j} f(u)=a_{j 0}(u) f(u)+\sum_{i=1}^{3} a_{j i}(u) R_{i} f(u)
$$

for any $f \in C^{\infty}(S U(2)) \cap L_{m}^{2}(S U(2))$, where $R_{i}$ stands for the same as in Lemma 1.7 .

Proof. Recall that $V(g) f(u)=\alpha(u g) / \alpha(u \bar{g}) f(u \bar{g})$. Here $\alpha(g)=\left|g_{22}\right|^{i_{\rho}-m-2}$ $\times g_{22}^{m}$ for $g=\left(g_{i j}\right) \in G$, and $u \bar{g}$ denotes a unitary representative of the coset $K u g, K$ being a subgroup $\left\{\left(\begin{array}{cc}\lambda^{-1} & \xi \\ 0 & \lambda\end{array}\right) ; \lambda>0\right\}$. Both maps $(u, g) \rightarrow$ $\alpha(u g) / \alpha(u \bar{g})$ and $(u, g) \rightarrow u \bar{g}$ defined on $S U(2) \times G$ are smooth. Indeed, first, simple calculation yields

$$
\alpha(u g) / \alpha(u \bar{g})=\left\{\left|u_{21} g_{11}+u_{22} g_{21}\right|^{2}+\left|u_{21} g_{12}+u_{22} g_{22}\right|^{2}\right\}^{(-2+i \rho-m) / 2}
$$

[12, p. 141]. Secondly, since a map $g \rightarrow e \bar{g}$ defined on $G$ is smooth [12, p. 141], $u \bar{g}$ is smooth on $S U(2) \times G$. Consequently $V\left(\omega_{j}(t)\right) f(u)$ is smooth on $R \times S U(2)$, from which the lemma follows at once.

Q.E.D. 
We return to the

Proof of Proposition 1.6. Set $C_{m}^{\infty}=C^{\infty}(S U(2)) \cap L_{m}^{2}(S U(2))$. As we noticed in the proof of Lemma 1.8, $V(g)$ leaves $C_{m}^{\infty}$ invariant. Recall that $\left\{\phi_{m / 2, \mu}^{k} \in C_{m}^{\infty} ; k=m / 2, m / 2+1, \cdots, \mu=-k,-k+1, \cdots, k\right\}$ is a complete orthogonal basis of $L_{m}^{2}(S U(2))$ and that, if $f_{\mu}^{k}$ and $\dot{\omega}_{j}$ in Proposition 1.6 are replaced by $\phi_{m / 2, \mu}^{k}$ and $\omega_{j}$ (see Lemma 1.8) respectively, still the equalities there hold [12, p. 147]. Denote by $C_{m, c}^{\infty}$ the algebraic linear span of $\left\{\phi_{m / 2, \mu}^{k}\right\}$. To prove the proposition, now it is enough to show that $i \omega_{j}$ restricted to $C_{m, c}^{\infty}$ is essentially selfadjoint. We denote this operator by $i \omega_{j, c}$. To this end we shall establish first the essentially selfadjointness of $i \omega_{j}$ restricted to $C_{m}^{\infty}$. We denote this operator by $i \omega_{j, \infty}$. Assume that an $f$ is orthogonal to the image $\left(\omega_{j}-\alpha\right) C_{m}^{\infty}(\operatorname{Re} \alpha \neq 0)$. Then, since $V(g) C_{m}^{\infty} \subset C_{m}^{\infty}$, we have $\left\langle f, V\left(\omega_{j}(t)\right)\left(\omega_{j}-\alpha\right) \phi\right\rangle=0$ for any $\phi \in C_{m}^{\infty}$. Multiplying both side by $e^{-\alpha t}$ and integrating on $R_{+}$or $R \backslash R_{+}$according as $\operatorname{Re} \alpha>0$ or not, we obtain $\langle f, \phi\rangle=0$, namely $f=0$. Secondly, the closure of $i \omega_{j, c}$ is an extention of $i \omega_{j, \infty}$ in virtue of Lemmas 1.7 and 1.8. Therefore $i \omega_{j, c}$ is essentially selfadjoint.

Q.E.D.

$\S 2$. Invariant subspaces common to $L_{k, \ell}, M_{k, \ell}$ and $T_{t}(t \geq 0)$ in $L^{2}(R)^{2 k+1}, k \in Z_{+}+1 / 2$

The ultimate aim of this section and the next one is to enumerate all non-trivial sequences $\left\{D_{k}\right\}_{k \in Z_{++}}$satisfying the following conditions (Q.1) and (Q.2);

(Q.1) $D_{k}$ is a closed subspace of $L^{2}(R)^{2 k+1}$, and is invariant under the selfadjoint operators $L_{k, \ell}, M_{k, \ell}$ and the semigroup $T_{t}(t \geq 0)$.

(Q.2) $K_{+, k, \ell} D_{k} \subset D_{k+1}$ and $K_{+, k-1, \ell}^{*} D_{k} \subset D_{k-1}$, where the domains of $K_{+, k, \ell}$ and $K_{+, k-1, \ell}^{*}$ are $H_{2}(R)^{2 k+1}$.

See (1.13) (1.14), (1.23) and (1.24) for the definition of the operators mentioned above. A sequence $\left\{D_{k}\right\}$ is said to be nontrivial if not all $D_{k}$ are trivial. To attain this aim, the following relations will be extensively used, the proof of which relies on direct calculation.

$$
\begin{aligned}
& K_{+, k, \ell} L_{k, \ell}=L_{k+1, \ell} K_{+, k, \ell}, \quad K_{+, k, \ell} M_{k, \ell}=M_{k+1, \ell} K_{+, k, \ell} . \\
& K_{+, k, \ell}^{*} K_{+, k, \ell}=4(k+1)^{4}+4(k+1)^{2} L_{k, \ell}-M_{k, \ell}^{2} . \\
& K_{+, k-1, \ell} K_{+, k-1, \ell}^{*}=4 k^{4}+4 k^{2} L_{k, \ell}-M_{k, \ell}^{2} .
\end{aligned}
$$

In the above the equalities holds on $C^{\infty}(R)^{2 k+1}$, and the left side of (2.3) is understood to be zero if $k \leq 1 / 2$. It also should be noted that $D_{k}$ is 
invariant under $T_{t}(t \geq 0)$ iff it is invariant under multiplication operators $G_{\alpha}=(\alpha-i \operatorname{sh} \tau)^{-1}(\operatorname{Re} \alpha>0)$. By abuse of notation $G_{\alpha}$ will sometimes stands for the function $(\alpha-i \operatorname{sh} \tau)^{-1}$ on $R$.

Throughout the rest of this section, assume that $k \in Z_{+}+1 / 2$ and $\ell$ $=-1 / 2+i \eta(\eta>0)$, and the suffix $\ell$ will be dropped in principle. Let us start with reviewing the eigenfunction expansion theorem for $M_{k}$. By the theorem $M_{k}$ will be reduced to a simpler operator, as far as invariant subspaces for $M_{k}$ are concerned. Denote by $\Phi_{k}(\tau, \lambda)$ the solution of a differential equation

$$
\left(M_{k}-\lambda\right) \Phi_{k}=0, \quad \Phi_{k}(0, \lambda)=I_{2 k+1} .
$$

Since $V_{k} / \operatorname{ch} \tau$ is integrable, there exists a so-called spectral density $\tilde{\rho}_{k}$ satisfying the following conditions i) iii) [4, Theorem 2].

i) $\tilde{\rho}_{k}$ is a $M_{2 k+1}^{++}$-valued continuous function on $R$.

ii) A map $\tilde{\mathscr{F}}_{k}: L^{2}(R)^{2 k+1} \rightarrow L^{2}\left(R, \tilde{\rho}_{k}\right)$ defined by

$$
\tilde{\mathscr{F}}_{k} f(\lambda)=\underset{N \rightarrow \infty}{\lim } \int_{|\tau|<N} \Phi_{k}^{*}(\tau, \lambda) f(\tau) d \tau
$$

is an onto isometry, whose inverse $\tilde{\mathscr{F}}_{k}^{-1}$ is given by

$$
\tilde{\mathscr{F}}_{k}^{-1} g(\tau)=\operatorname{li}_{N \rightarrow \infty} . \int_{|\lambda|<N} \Phi_{k}(\tau, \lambda) \tilde{\rho}_{k}(\lambda) g(\lambda) d \lambda .
$$

iii) $\tilde{\mathscr{F}}_{k} M_{k} \tilde{\mathscr{F}}_{k}^{-1} g(\lambda)=\lambda g(\lambda)$ if $\lambda g(\lambda) \in L^{2}\left(R, \tilde{\rho}_{k}\right)$.

Denote by $\bar{M}_{k}$ a differential operator $2 i A_{k} d / d \tau+\bar{V}_{k} / \mathrm{ch} \tau$, and consider the following equations;

$$
\left(M_{k}-\lambda\right) \zeta=0 \text {. }
$$

$$
\left(\bar{M}_{k}-\lambda\right) \chi=0
$$

Both of them have regular singularity at $\tau=i \pi / 2$, in other words at $\sigma=0$. By definition $\alpha$ is an indicial root of the equation $\overline{(2.6)}$ at $\sigma=0$ if $\operatorname{det}\left(\alpha-2^{-1} A_{k}^{-1} \bar{V}_{k}\right)=0$. The definition of an indicial root of the equation (2.6) is similar.

Lemima 2.1. The sets of indicial roots of (2.6) and $\overline{(2.6)}$ coincide. They are $\left\{\alpha_{k, \nu} ; \nu=-k,-k+1, \cdots, k\right\}$, where

$$
\alpha_{k, \nu}=-(k+1 / 2)+(\operatorname{sign} \nu)(2 \nu+i \eta) .
$$

Proof. The complex conjugate of an indicial root of one equation is the one of the other. So only the equation (2.6) will be discussed. If $k=1 / 2$, the lemma holds. Suppose the assertion is valid up to $k$. 
It suffices to show that $-(k+3 / 2)+2 \nu \pm i \eta(\nu=1 / 2,3 / 2, \cdots, k)$ are characteristic roots of $-\left(2 A_{k+1}\right)^{-1} V_{k+1}$. Indeed, since the characteristic polynomial of the matrix is even, $-(k+3 / 2)+2 \nu \pm i \eta(\nu=k+1)$ turn out to be characteristic roots. To complete the proof, assume that $\left\{-\left(2 A_{k}\right)^{-1} V_{k}-\alpha_{k, \nu}\right\} p_{k, \nu}=0(|\nu| \leq k)$ for $p_{k, \nu} \in M_{2 k+1,1} \mid\{0\} . \quad$ By Lemma A.2 [7] the equation (2.6) has solutions $\phi_{k, \nu}$ assuming the form $\sigma^{\alpha_{k, \nu}}\left(p_{k, \nu}+\sigma h(\sigma, \log \sigma)\right)$ near $\sigma=0$. Since $K_{+, k} \phi_{k, \nu}$ takes the form $\sigma^{\alpha_{k, \nu}-1}\left(p_{k+1, \nu}+\sigma h(\sigma, \log \sigma)\right)$ for some non-zero vector $p_{k+1, \nu}, \alpha_{k, \nu}-1$ is a characteristic root of $-\left(2 A_{k+1}\right)^{-1} V_{k+1}$ on account of Lemma A.2 [7].

Q.E.D.

When $k=1 / 2$, all invariant proper closed subspaces common to $M_{k}$ and $T_{t}(t \geq 0)$ can be specified. We shall define the subspaces. To begin with, by Lemma A.2 [7] there are solutions $\zeta_{k, \pm k}(\tau, \lambda)$ of (2.6) and $\chi_{k, \pm k}(\tau, \lambda)$ of (2.6) which, being holomorphic in $\dot{D}_{\tau} \times C$, take the following form near $\sigma=0$;

$$
\zeta_{k, \pm k}=\sigma^{ \pm i \eta}\left(\sum_{n=0}^{\infty} z_{k, \pm k, n} \sigma^{n}\right), \quad \chi_{k, \pm k}=\sigma^{ \pm i \eta}\left(\sum_{n=0}^{\infty} x_{k, \pm k, n} \sigma^{n}\right),
$$

where ${ }^{t} z_{k, \pm k, 0}={ }^{t} x_{k, \pm k, 0}=(1, \mp 1)$. Set $\zeta_{k}=\left(\zeta_{k,-k}, \zeta_{k, k}\right), \chi_{k}=\left(\chi_{k,-k}, \chi_{k, k}\right)$, and define $Z_{k}, X_{k}, s_{k, \pm}$ and $r_{k, \pm}$ by

$$
\begin{aligned}
& \zeta_{k}(\tau, \lambda)=\Phi_{k}(\tau, \lambda) Z_{k}(\lambda), \quad \chi_{k}(\tau, \lambda)=\bar{\Phi}_{k}(\tau, \lambda) X_{k}(\lambda) \text { for }(\tau, \lambda) \in R^{2}, \\
& s_{k, \pm}=X_{k}^{t}(1 \pm 1,1 \mp 1), \quad r_{k, \pm}=\left(\begin{array}{rr}
0 & 1 \\
-1 & 0
\end{array}\right) s_{k, \pm} \quad(k=1 / 2) .
\end{aligned}
$$

In terms of the isometry $\tilde{F}_{1 / 2}$ we define proper closed subspaces $D_{1 / 2, \pm}^{e}$ of $L^{2}(R)^{2}$ by

$$
D_{1 / 2, \pm}^{\ell}=\tilde{\mathscr{F}}_{1 / 2}^{-1}\left\{g \in L^{2}\left(R, \tilde{\rho}_{1 / 2}\right) ;{ }^{t} s_{1 / 2, \pm}(\lambda) g(\lambda)=0 \text { a.e. }\right\} \text {. }
$$

These subspaces are what we intended to define, for we can show

THEOREM 2.1. Let $D$ be a closed proper subspace of $L^{2}(R)^{2}$.

(i ) $D$ is invariant under the selfadjoint operator $M_{1 / 2, \ell}$ and $T_{t}(t \geq 0)$ iff it coincides with one of $D_{1 / 2, \pm}^{\ell}$.

(ii) $D_{1 / 2, \pm}^{\ell}$ are invariant under the selfadjoint operator $L_{1 / 2, \ell}$.

For the proof we prepare a few lemmas in advance. The first one is concerned with estimates of the solutions of (2.6) and (2.6).

LEMMA 2.2.

(i) Fix $\lambda_{0} \in R$ and $\varepsilon>0$. Then there exist positive $K$ and $\delta$ such that 
$\left|\Phi_{k}\left(\tau, \lambda_{0}\right)\right|<K$ on $\bar{D}_{\tau} \cap\{\operatorname{Re} \tau \mid \geq 1\},\left|\Phi_{k}(\tau, \lambda)\right|<K e^{\varepsilon|\tau|}$ on $R \times\left\{\left|\lambda-\lambda_{0}\right|<\delta\right\}$.

(ii) Let $\chi(\tau, \lambda)$ be a solution of $\overline{(2.6)}$ with $\chi(0, \lambda)=I_{2 k+1}$. Then the statement (i) holds for $\chi$.

Proof. Put $\Psi=\exp \left\{\lambda \tau\left(2 i A_{k}\right)^{-1}\right\} \Phi_{k}$. Then $\Psi$ satisfies an equation $\Psi^{\prime}=$ $W(\tau, \lambda) \Psi$ with $\Psi(0, \lambda)=I_{2 k+1}$, where $W=\exp \left\{\lambda \tau\left(2 i A_{k}\right)^{-1}\right\}\left(2 i A_{k}\right)^{-1} V_{k} \exp \{-\lambda \tau$ $\left.\times\left(2 i A_{k}\right)^{-1}\right\} / \operatorname{ch} \tau$. Trivially $\left|\Psi\left(\tau, \lambda_{0}\right)\right|$ is bounded on $\bar{D}_{\varepsilon} \cap\{|\operatorname{Re} \tau|=1\}$. Moreover, there exists an integrable function $w$ on $(-\infty,-1] \cup[1, \infty)$ such that $\left|W\left(\tau, \lambda_{0}\right)\right|<w(\operatorname{Re} \tau)$ on $\bar{D}_{\tau} \cap\{|\operatorname{Re} \tau| \geq 1\}$. It now follows from Problem 1 $\left[2\right.$, p. 97] that $\left|\Psi\left(\tau, \lambda_{0}\right)\right|$, hence $\left|\Phi_{k}\left(\tau, \lambda_{0}\right)\right|$ as well, is bounded on $\bar{D}_{\tau} \times\{|\operatorname{Re} \tau|$ $\geq 1\}$. Next take so small a positive $\delta$ that $\left|\operatorname{Im}\left\{\lambda\left(2 A_{k}\right)^{-1}\right\}\right|<\min \{\varepsilon, 1 / 4\}$. Then there is an integrable function $v$ on $R$ such that $|W(\tau, \lambda)|<v(\tau)$ on $R \times\left\{\lambda-\lambda_{0} \mid<\delta\right\}$. Using Problem 1 [2, p. 97], we conclude that $|\Psi(\tau, \lambda)|$ is bounded on $R \times\left\{\left|\lambda-\lambda_{0}\right|<\delta\right\}$. Now the second inequality in (i) follows at once. The proof of (ii) is quite similar to that of (i). Q.E.D.

Denote by $M_{k}(\sigma)$ and $\bar{M}_{k}(\sigma)$ the differential operators $M_{k}$ and $\bar{M}_{k}$ represented in terms of $\sigma=\tau-i \pi / 2$ respectively, and let $R_{k}$ be a map sending an $M_{2 k+1,1}$-valued function $f(\sigma)={ }^{t}\left(f_{k}(\sigma), \cdots, f_{-k}(\sigma)\right)$ to ${ }^{t}\left(f_{-k}(-\sigma)\right.$, $\left.\cdots, f_{k}(-\sigma)\right)$.

LEMMA 2.3.

(i ) $R_{k} M_{k}(\sigma) R_{k}=M_{k}(\sigma), \quad R_{k} \bar{M}_{k}(\sigma) R_{k}=\bar{M}_{k}(\sigma), \quad R_{k} L_{k}(\sigma) R_{k}=L_{k}(\sigma)$, $R_{k} \bar{L}_{k}(\sigma) R_{k}=\bar{L}_{k}(\sigma)$.

(ii) For $k=1 / 2$ and $n \in Z_{+}$, we have

$$
R_{k} z_{k, \pm k, n}=\mp(-1)^{n} z_{k, \pm k, n}, \quad R_{k} x_{k, \pm k, n}=\mp(-1)^{n} x_{k, \pm k, n} .
$$

Proof. The assertion (i) is easy to verify. As to (ii) only $z_{k, \pm k, n}$ will be treated. Set $z_{k, \pm k}(\sigma)=\sum_{n=0}^{\infty} z_{k, \pm k, n} \sigma^{n}$. Then it is enough to show that $R_{k} z_{k, \pm k}(\sigma)=\mp z_{k, \pm k}(\sigma)$. To this end notice that $R_{k} \zeta_{k, \pm k}=a_{ \pm k} \zeta_{k, \pm k}$ for some constant $a_{ \pm k}$ on account of (2.1). Consequently $R_{k} z_{k, \pm k}(\sigma)=b_{ \pm k} z_{k, \pm k}(\sigma)$ for some constant $b_{ \pm k}$, which yields $b_{ \pm k}=\mp 1$, since ${ }^{t} z_{k, k, 0}=(1, \mp 1)$. Q.E.D.

The following lemma is concerned with the if part of Theorem 2.1.

Lemma 2.4. Let $k=1 / 2$ and $\nu= \pm 1 / 2$. Then, for any reals $\lambda$, $\xi$ and $\alpha(\operatorname{Re} \alpha>0)$, the integral $\left\langle{ }^{t} \chi_{k, \nu}(\tau, \lambda) G_{\alpha} \zeta_{k,-\nu}(\tau, \xi)\right\rangle$ vanishes.

Proof. By Lemma 2.3 (ii) ${ }^{t} \chi_{k, \nu}(\tau, \lambda) \zeta_{k,-\nu}(\tau, \xi)$ takes the form $\sum_{n=0}^{\infty} c_{n} \sigma^{2 n+1}$ near $\sigma=0$. Changing the variable $\tau$ to $z=(1+i \operatorname{sh} \tau) / 2$, we can apply 
Proposition 1.2 (i) [7] to the integral in view of Lemma 1.1 (ii) [7] and Lemma 2.2.

Q.E.D.

We return to the

Proof of Theorem 2.1. Let $k=1 / 2$ throughout the proof. 1) We shall show that $D_{k, \pm}^{e}$ have the desired invariant property. By the aid of the isometry $\tilde{\mathscr{F}}_{k}$ it is easy to see that they are $M_{k}$-invariant. Since $L_{k}$ $=M_{k}^{2}-1 / 4$ by (2.3), they are $L_{k}$-invariant too. In order to show that $T_{t}(t \geq 0)$ leaves $D_{k, \pm}^{e}$ invariant, it suffices to verify

$$
{ }^{t} s_{k, \pm}(\lambda)\left[\tilde{\mathscr{F}}_{k} G_{\alpha} \tilde{\mathscr{F}}_{k}^{-1} r_{k, \pm} h\right](\lambda)=0 \text { for } \operatorname{Re} \alpha>0 \text { and } h \in C_{0}(R)^{1} .
$$

To this end we will show

$$
\left\langle{ }^{t} \boldsymbol{s}_{k, \pm}(\lambda) \Phi_{k}^{*}(\tau, \lambda) G_{\alpha} \Phi_{k}(\tau, \xi) \tilde{\rho}_{k}(\xi) r_{k, \pm}(\xi)\right\rangle=0,
$$

from which (2.10) follows immediately. Put

$$
I_{\alpha, \lambda, \xi}=\left\langle{ }^{t} \chi_{k}(\tau, \lambda) G_{\alpha} \zeta_{k}(\tau, \xi)\right\rangle, \quad \rho=Z_{k}^{-1} \tilde{\rho}_{k}{ }^{t} X_{k}^{-1}, \quad v_{ \pm}={ }^{t}(1 \pm 1,1 \mp 1) .
$$

Then the left side of the equality (2.11) can be rewritten as ${ }^{t} v_{ \pm} I_{\alpha, \lambda, \xi} \rho(\xi) \times$ $\left(\begin{array}{rr}0 & 1 \\ -1 & 0\end{array}\right) v_{ \pm} \operatorname{det} X_{k}(\xi)$, because $\left(\begin{array}{rr}0 & 1 \\ -1 & 0\end{array}\right) Y\left(\begin{array}{rr}0 & 1 \\ -1 & 0\end{array}\right)=-{ }^{t} Y^{-1} \operatorname{det} Y$ for a regular matrix $Y$. On the other hand, since $I_{\alpha, \lambda, \xi}$ is diagonal by Lemma 2.4, there results that $\rho(\xi)$ is also diagonal (see the proof of Theorem 1.1 [7]). Now one can verify (2.11) easily. 2) We shall show that if $M_{k}$ and $T_{t}(t \geq 0)$ keep $D$ invariant, then $D=D_{k, \pm}^{\ell}$. According to Proposition 1.4 [7] there exist disjoint Borel sets $B_{1}, B_{2}$ of $R$ and a Borel measurable function $s$ on $B_{1}$ with values in $M_{2,1} \mid\{0\}$ such that

$$
\begin{aligned}
\tilde{\mathscr{F}}_{k} D= & \left\{g \in L^{2}\left(R, \tilde{\rho}_{k}\right) ; g=0 \text { a.e. outside } B_{1} \text {, and }{ }^{t} s g=0 \text { a.e. on } B_{1}\right\} \\
& \oplus\left\{g \in L^{2}\left(R, \tilde{\rho}_{k}\right) ; g=0 \text { a.e. outside } B_{2}\right\} .
\end{aligned}
$$

We must show that $B_{1}=R, B_{2}=\phi$ and $s=c_{ \pm} s_{k, \pm}$ for some $C^{*}$-valued measurable function $c_{ \pm}$on $R$. Since $G_{\alpha} D$ is dense in $D$, there exist $f_{1}$ and $f_{2}$ in $D$ such that

$$
\operatorname{det}\left(\tilde{\mathscr{F}}_{k} G_{\alpha} f_{1}(\lambda), \tilde{\mathscr{F}}_{k} G_{\alpha} f_{2}(\lambda)\right) \neq 0 \quad \text { a.e. on } B_{2} \text {. }
$$

If $B_{2}$ is not a null set, the determinant does not vanish a.e. on $R$, for it is real analytic in $\lambda$ by virtue of Lemma 2.2. Since $D$ is a proper closed subspace, $B_{2}$ must be a null set. Now that $B_{2}=\phi$, analyticity of $\tilde{\mathscr{F}}_{k} G_{\alpha} f$ $(f \in D \backslash\{0\})$ yields $B_{1}=R$. Moreover we can assume $s=\left(\begin{array}{rr}0 & 1 \\ -1 & 0\end{array}\right) \widetilde{\mathscr{F}}_{k} G_{\alpha} f$. Put $r=\left(\begin{array}{rr}0 & 1 \\ -1 & 0\end{array}\right) s$. Then, for any $h \in C_{0}(R)^{1}$ and $\lambda \in R$, we have 


$$
{ }^{t} s(\lambda)\left[\tilde{\mathscr{F}}_{k} G_{\alpha} \tilde{\mathscr{F}}_{k}^{-1} r_{ \pm} h\right](\lambda)=0 .
$$

Let $h$ converge to the Dirac measure supported at $\xi$ to obtain $\left\langle{ }^{t} s(\lambda) \Phi_{k}^{*}(\tau, \lambda)\right.$ $\left.\times G_{\alpha} \Phi_{k}(\tau, \xi) \tilde{\rho}_{k}(\xi) r(\xi)\right\rangle=0$, which is equivalent to

$$
{ }^{t}\left(X_{k}^{-1} s\right)(\lambda) I_{\alpha, \lambda, \xi} \rho(\xi)\left(\begin{array}{rr}
0 & 1 \\
-1 & 0
\end{array}\right)\left(X_{k}^{-1} s\right)(\xi)=0 .
$$

Put $X_{k}^{-1} s={ }^{t}\left(a_{-k}, a_{k}\right)$ and $\rho_{\nu}=(\nu, \nu)$-component of $\rho(\nu= \pm k)$. Then (2.14) implies on account of Proposition 1.2 (ii) [7] that a function of $z=(1+$ $i \operatorname{sh} \tau) / 2$

$$
\sum_{\nu= \pm k} a_{\nu}(\lambda)^{t} \chi_{k, \nu}(\tau, \lambda) \zeta_{k, \nu}(\tau, \xi) \rho_{\nu}(\xi) a_{-\nu}(\xi) /\{z(1-z)\}^{1 / 2}
$$

is holomorphic in $\{\operatorname{Re} z<1\}$. Thanks to Lemma 1.1 [7], the function is so iff $a_{\nu}(\lambda) a_{-\nu}(\xi)=0(\nu= \pm k)$. Since $a_{\nu}$ is real analytic, it follows that either $a_{k}=0$ or $a_{-k}=0$. That is, $s=c_{ \pm} s_{k, \pm}$.

Q.E.D.

When $k>1 / 2$, there are, as will be shown later, at least two proper closed subspaces of $L^{2}(R)^{2 k+1}$, say $D_{k, \pm}^{\ell}$, satisfying the condition (Q.1). For our purpose it is desirable, but not necessary, to determine all closed proper subspaces satisfying the condition. In order to define $D_{k, \pm}^{\ell}$ we begin with

Lemma 2.5. Let $\zeta={ }^{t}\left(\zeta_{k}, \cdots, \zeta_{-k}\right)$ and $\chi={ }^{t}\left(\chi_{k}, \cdots, \chi_{-k}\right)$ be solutions of (2.6) and (2.6) respectively. Then $\zeta$ and $\chi$ satisfy

$$
K_{+, k-1}^{*} \zeta=0, \quad \overline{(2.15)} \quad \bar{K}_{+, k-1}^{*} \chi=0,
$$

respectively iff $\zeta_{k}$ and $\chi_{k}$ solve certain second order differential equations of the following form respectively.

$$
\begin{aligned}
\sum_{n=0}^{2} \sigma^{n} c_{k, 2-n}(\sigma, \lambda) \zeta_{k}^{(n)} & =0, & c_{k, 0} & =1, \\
\sum_{n=0}^{2} \sigma^{n} \tilde{c}_{k, 2-n}(\sigma, \lambda) \chi_{k}^{(n)} & =0, & \tilde{c}_{k, 0} & =1,
\end{aligned}
$$

where $c_{k, n}$ and $\tilde{c}_{k, n}$, being holomorphic in $\dot{D}_{\tau} \times C$, satisfy the following condition;

$$
\begin{aligned}
\alpha(\alpha-1)+c_{k, 1}(0, \lambda) \alpha+c_{k, 2}(0, \lambda) & =\alpha(\alpha-1)+\tilde{c}_{k, 1}(0, \lambda) \alpha+\tilde{c}_{k, 2}(0, \lambda) \\
& =\left(\alpha-\alpha_{k,-1 / 2}\right)\left(\alpha-\alpha_{k, 1 / 2}\right) .
\end{aligned}
$$

Proof. The $\nu$-component of $\zeta$ is expressible in terms of $\zeta_{k}$ and its 
derivatives. Suppose $\zeta$ satisfies (2.15). Then the equality $\left(K_{+, k-1}^{*} \zeta\right)_{k-1}=0$ gives a second order differential equation (2.16). In particular, it follows easily that $\operatorname{dim}\left(\operatorname{Ker} K_{+, k-1}^{*}\right)=2$, where $K_{+, k-1}^{*}$ is regarded as a linear map sending solutions of (2.6) to solutions of (2.6) with the suffix $k-1$. Therefore, if $\zeta_{k}$ solves (2.16), $\zeta$ satisfies (2.15). Similar argument is available for $\chi$.

At this stage fundamental systems $\left\{\psi_{k, \nu} ;|\nu|=1 / 2, \cdots, k\right\}$ and $\left\{\tilde{\psi}_{k, \nu}\right.$; $|\nu|=1 / 2, \cdots, k\}$ of the equations (2.6) and (2.6) respectively are to be introduced.

$$
\psi_{k, \nu}=\left\{\begin{array}{l}
\zeta_{1 / 2, \nu} \text { for } k=|\nu|=1 / 2, \\
K_{+, k-1} \psi_{k-1, \nu}(|\nu|<k) \text { or } \psi_{k, \pm k}(|\nu|=k),
\end{array}\right.
$$

where $\psi_{k, \pm k}(\tau, \lambda)$, being holomorphic in $\dot{D}_{\tau} \times C$, satisfy $K_{+, k-1}^{*} \psi_{k, \pm k}=0$ and have the following expansion near $\sigma=0$;

$$
\begin{aligned}
& \psi_{k, \pm k}=\sigma^{\alpha k, \pm 1 / 2}\left(\sum_{n=0}^{\infty} e_{k, \pm k, n} \sigma^{n}\right), \quad e_{k, \pm k, 0} \neq 0 . \\
& \tilde{\psi}_{k, \nu}=\left\{\begin{array}{l}
\chi_{1 / 2, \nu} \text { for } k=|\nu|=1 / 2, \\
\bar{K}_{+, k-1} \tilde{\psi}_{k-1, \nu}(|\nu|<k) \text { or } \tilde{\psi}_{k, \pm k}(|\nu|=k),
\end{array}\right.
\end{aligned}
$$

where $\tilde{\psi}_{k, \pm k}(\tau, \lambda)$, being holomorphic in $\dot{D}_{\tau} \times C$, satisfy $\bar{K}_{+, k-1}^{*} \tilde{\psi}_{k, \pm k}=0$ and have the following form;

$$
\tilde{\psi}_{k, \pm k}=\sigma^{\alpha k, \pm 1 / 2}\left(\sum_{n=0}^{\infty} \tilde{e}_{k, \pm k, n} \sigma^{n}\right), \quad \tilde{e}_{k, \pm k, 0} \neq 0 .
$$

Note that $\psi_{k, \pm k}$ as well as $\tilde{\psi}_{k, \pm k}$ really exists in view of Lemma A.1 [7] and Lemma 2.5. Put $\Psi_{k, \nu}=\left(\psi_{k,-\nu}, \psi_{k, \nu}\right), \Psi_{k}=\left(\Psi_{k, 1 / 2}, \cdots, \Psi_{k, k}\right), \tilde{\Psi}_{k, \nu}=\left(\psi_{k,-\nu}\right.$, $\left.\tilde{\psi}_{k, \nu}\right)$, and define $Z_{k}(\lambda), \rho_{k}(\lambda) \in M_{2 k+1}, X_{k}(\lambda) \in M_{2}$ and $s_{k, \pm}(\lambda), r_{k, \pm}(\lambda) M_{2,1}$ by

$$
\begin{array}{ll}
\Phi_{k}(\tau, \lambda)=\Psi_{k}(\tau, \lambda) Z_{k}(\lambda), & \rho_{k}=Z_{k} \tilde{\rho}_{k} Z_{k}^{*}, \\
\tilde{\Psi}_{k, k}(\tau, \lambda)=\bar{\Psi}_{k, k}(\tau, \lambda) X_{k}(\lambda) & \text { for }(\tau, \lambda) \in R^{2}, \\
s_{k, \pm}=X_{k}{ }^{t}(1 \pm 1,1 \mp 1), & r_{k, \pm}=\left(\begin{array}{rr}
0 & 1 \\
-1 & 0
\end{array}\right) s_{k, \pm}
\end{array}
$$

Via an onto isometry: $L^{2}\left(R, \tilde{\rho}_{k}\right) \rightarrow L^{2}\left(R, \rho_{k}\right)$ sending $g$ to $Z^{*-1} g$ there arise an onto isometry $\mathscr{F}_{k}: L^{2}(R)^{2 k+1} \rightarrow L^{2}\left(R, \rho_{k}\right)$ and its inverse $\mathscr{F}_{k}^{-1}$;

$$
\begin{aligned}
\mathscr{F}_{k} f(\lambda) & =\underset{N \rightarrow \infty}{\lim .} \int_{|\tau|<N} \Psi_{k}^{*}(\tau, \lambda) f(\tau) d \tau . \\
\mathscr{F}_{k}^{-1} g(\tau) & =\operatorname{li} \lim _{N \rightarrow \infty} \int_{|\lambda|<N} \Psi_{k}(\tau, \lambda) \rho_{k}(\lambda) g(\lambda) d \lambda .
\end{aligned}
$$


The eigenfunction expansion for $M_{k}$ with respect to $\Psi_{k}$ is more convenient in the sense that the spectral density $\rho_{k}$ has a simpler form.

Proposition 2.6.

(i ) $\rho_{k}(\lambda)=\left(\begin{array}{ccc}\rho_{k, 1 / 2}(\lambda) & & 0 \\ & \rho_{k, 3 / 2}(\lambda) & \\ 0 & & \rho_{k, k}(\lambda)\end{array}\right), \rho_{k, \nu}(\lambda) \in M_{2}^{++}$.

(ii) $\rho_{k, \nu}=n_{k, \nu} \rho_{k-1, \nu}$ for $\nu<k$, where $n_{k, \nu}(\lambda)=\left\{\left(k^{2}-\nu^{2}\right)\left(4 k^{2}+\lambda^{2} / \nu^{2}\right)\right\}^{-1}$.

Proof. (i) Suppose the assertion (i) is true up to $k-1(k>1 / 2)$. First putting $f_{\nu}=\int \Psi_{k, \nu} g_{\nu} d \lambda$ for $g_{\nu} \in C_{0}(R)^{2}(1 / 2 \leq \nu \leq k)$, we will show that $\left\langle f_{\nu}, f_{\nu^{\prime}}\right\rangle=0$ if $\nu \neq \nu^{\prime}$. To this end note that $f_{\nu}(\tau) \rightarrow 0$ as $\mid \tau^{\prime} \rightarrow \infty$ and that

$$
K_{+, k-1}^{*} K_{+, k-1} \Psi_{k-1, \nu}^{*}=\left\{\left(k^{2}-\nu^{2}\right)\left(4 k^{2}+\lambda^{2} / \nu^{2}\right)\right\}^{-1} \Psi_{k-1, \nu},
$$

which is an easy consequence of the fundamental relations $(2.1) \sim(2.3)$. Since $\left\langle f_{\nu}, f_{\nu^{\prime}}\right\rangle$ is equal to

$$
\lim _{\Lambda^{\prime} \rightarrow \infty} \int_{|\tau|<N}\left(K_{+, k-1}^{*} \Psi_{k, \nu} g_{\nu} d \lambda\right)^{*}\left(\Psi_{k-1, \nu^{\prime}} g_{\nu^{\prime}} d \lambda\right) d \tau
$$

for $\nu^{\prime}<k$, there results $\left\langle f_{\nu}, f_{\nu^{\prime}}\right\rangle=0$ if $\nu \neq \nu^{\prime}$. Secondly, denoting by $Q_{\nu}$ the matrix such that $Q_{\nu}{ }^{t}\left({ }^{t} g_{1 / 2}, \cdots,{ }^{t} g_{k}\right)={ }^{t}\left(0, \cdots 0,{ }^{t} g_{\nu}, 0 \cdots, 0\right)$, we will show that $\rho_{k}^{-1}=\sum_{\nu=1 / 2}^{k} Q_{\nu} \rho_{k}^{-1} Q_{\nu}$, from which (i) follows at once. In fact, for any $h \in C_{0}(R)^{2 k+1}$, we have

$$
\begin{aligned}
\int\left\langle g(\lambda), \rho_{k}(\lambda) h(\lambda)\right\rangle d \lambda & =\left\langle\mathscr{F}_{k}^{-1} g, \mathscr{F}_{k}^{-1} h\right\rangle \\
& =\sum_{\nu=1 / 2}^{k} \int\left\langle\rho_{k}^{-1} Q_{\nu} \rho_{k} g, Q_{\nu} \rho_{k} h\right\rangle d \lambda .
\end{aligned}
$$

(ii) Now $\left\langle f_{\nu}, f_{\nu}\right\rangle=\int\left\langle\rho_{k, \nu}^{-1} g_{\nu}, g_{\nu}\right\rangle d \lambda(\nu<k)$. The left side is equal to $\int\left\langle n_{k, \nu}^{-1} \rho_{k-1, \nu}^{-1} g_{\nu}, g_{\nu}\right\rangle d \lambda$ on account of (2.21) and (2.22). Since $g_{\nu} \in C_{0}(R)^{2}$ is arbitrary, (ii) has been proved.

Q.E.D.

We are in a position to define closed proper subspaces $D_{k, \pm}^{e}$ of $L^{2}(R)^{2 k+1}$.

$$
D_{k, \pm}^{\ell}=\mathscr{F}_{k}^{-1}\left\{\left(g_{\nu}\right) \in L^{2}\left(R, \rho_{k}\right) ;{ }^{t} s_{\nu, \pm}(\lambda) g_{\nu}(\lambda)=0 \text { a.e. }\right\} .
$$

Our main result in this section is the following theorem.

Theorem 2.2. Let $D_{k}$ be a closed subspace of $L^{2}(R)^{2 k+1}$ for each $k \in Z_{+}$ $+1 / 2$. Then the sequence $\left\{D_{k}\right\}$ is nontrivial and satisfies the conditions 
(Q.1) and (Q.2) (see the beginning of $\S 2$ ) iff it coincides with either $\left\{D_{k,-}^{e}\right\}$ or $\left\{D_{k,+}^{\ell}\right\}$. The sequence is said to be nontrivial if not all $D_{k}$ are trivial.

Before going into the proof we shall compile some facts. To begin with, let us introduce other fundamental systems $\left\{\zeta_{k, \nu} ;|\nu|=1 / 2, \cdots, k\right\}$ and $\left\{\chi_{k, \nu} ;|\nu|=1 / 2, \cdots, k\right\}$ of the equations (2.6) and (2.6) respectively as follows.

$$
\zeta_{k, \nu}=K_{+, k-1} \zeta_{k-1, \nu}(|\nu|<k) \text { or } \zeta_{k, \nu}(|\nu|=k),
$$

where $\zeta_{k, \pm k}(\tau, \lambda)$, being holomorphic in $\dot{D}_{\tau} \times C$, have the following expression near $\sigma=0$;

$$
\begin{array}{ll}
\zeta_{k, \pm k}=\sigma^{\alpha k, \pm k}\left(\sum_{n=0}^{\infty} z_{k, \pm k, n} \sigma^{n}\right), & z_{k, \pm k, 0} \neq 0 . \\
\chi_{k, \nu}=\bar{K}_{+, k-1} \chi_{k-1, \nu}(|\nu|<k) & \text { or } \quad \chi_{k, \nu}(|\nu|=k),
\end{array}
$$

where $\chi_{k, \pm k}(\tau, \lambda)$, being holomorphic in $\dot{D}_{\tau} \times C$, assume the following form near $\sigma=0$;

$$
\chi_{k, \pm k}=\sigma^{\alpha k, \pm k}\left(\sum_{n=0}^{\infty} x_{k, \pm k, n} \sigma^{n}\right), \quad x_{k, \pm k, 0} \neq 0 .
$$

As to the definition of $\zeta_{k, \nu}$ and $\chi_{k, \nu}$ for $k=1 / 2$, see (2.7). Thanks to Lemma A.2 [7], $\zeta_{k, \pm k}$ and $\chi_{k, \pm k}$ are well-defined up to constant multiple. It is easy to see that $\zeta_{k, \nu}$ and $\chi_{k, \nu}$ have the form around $\sigma=0$;

$$
\begin{aligned}
\zeta_{k, \nu} & =\sigma^{\alpha k, \nu}\left(\sum_{n=0}^{\infty} z_{k, \nu, n} \sigma^{n}\right), & & z_{k, \nu, 0} \neq 0, \\
\chi_{k, \nu} & =\sigma^{\alpha k, \nu}\left(\sum_{n=0}^{\infty} x_{k, \nu, n} \sigma^{n}\right), & x_{k, \nu, 0} & \neq 0 .
\end{aligned}
$$

Recall the definition of the operator $R_{k}$ made above Lemma 2.3.

LEMMA 2.7.

(i ) $R_{k} z_{k, \nu, n}=-(-1)^{n}(\operatorname{sign} \nu) z_{k, \nu, n}, R_{k} x_{k, \nu, n}=-(-1)^{n}(\operatorname{sign} \nu) x_{k, \nu, n}$.

(ii) If $\nu \nu^{\prime}<0$ and $\alpha_{k, \nu}+\alpha_{k, \nu^{\prime}} \geq 0$, then

$$
\left\langle{ }^{t} \chi_{k, \nu}(\tau, \lambda) G_{\alpha} \zeta_{k, \nu^{\prime}}(\tau, \lambda)\right\rangle=0 \text { for }(\tau, \lambda) \in R^{2} \text { and } \alpha(\operatorname{Re} \alpha>0) \text {. }
$$

Proof. (i ) Put $z_{k, \nu}(\sigma)=\sum_{n=0}^{\infty} z_{k, \nu, n} \sigma^{n}$ and $x_{k, \nu}(\sigma)=\sum_{n=0}^{\infty} x_{k, \nu, n} \sigma^{n}$. We shall show that

$$
R_{k} z_{k, \nu}(\sigma)=-(\operatorname{sign} \nu) z_{k, \nu}(\sigma), \quad R_{k} x_{k, \nu}(\sigma)=-(\operatorname{sign} \nu) x_{k, \nu}(\sigma) .
$$

Only $x_{k, \nu}(\sigma)$ will be discussed. The relation (2.25) holds for $k=1 / 2$ by Lemma 2.3. Suppose (2.25) is valid up to $k$. First note that $R_{k+1} \bar{K}_{+, k}(\sigma)$ 
$R_{k}=-\bar{K}_{+, k}(\sigma)$, where $\bar{K}_{+, k}(\sigma)=-2 i B_{k}\{d / d \sigma-(k+1) \operatorname{coth} \sigma\}-\bar{Y}_{k} / \operatorname{sh} \sigma$, and apply $R_{k+1}$ to both sides of an identity $\chi_{k+1, \nu}=\bar{K}_{+, k} \chi_{k, \nu}(|\nu| \leq k)$ to obtain $(-\sigma)^{\alpha_{k+1, \nu}} R_{k+1} x_{k+1, \nu}(\sigma)=(-\sigma)^{\alpha_{k, \nu}}(\operatorname{sign} \nu) x_{k, \nu}(\sigma)$. Since $\alpha_{k+1, \nu}=\alpha_{k, \nu}$ -1 , (2.25) holds for the suffix $(k+1, \nu)(|\nu| \leq k)$. Secondly, note that $R_{k} \bar{K}_{+, k}^{*}(\sigma) R_{k+1}=-\bar{K}_{+, k}^{*}(\sigma)$ and that, for $|\nu|=k+1$,

$$
\begin{aligned}
& R_{k+1} \chi_{k+1, \nu}=c_{\nu} \chi_{k+1, \nu} . \\
& \bar{K}_{+, k}^{*} \chi_{k+1, \nu}=d_{\nu} \chi_{k, \nu^{\prime}}, \quad \nu^{\prime}=\nu-(\operatorname{sign} \nu) .
\end{aligned}
$$

Indeed, (2.26) follows from Lemma 2.3 (i) and (2.24) while (2.27) from (2.1) and Lemma 2.5. Now applying $R_{k}$ to the both sides in (2.27) and using (2.26), we obtain $c_{\nu} d_{\nu} \sigma^{\alpha_{k+1, \nu}} x_{k, \nu^{\prime}}(\sigma)=(\operatorname{sign} \nu) d_{\nu}(-\sigma)^{\alpha_{k, \nu^{\prime}}} x_{k, \nu^{\prime}}(\sigma)$, which yields $c_{\nu}=-(\operatorname{sign} \nu)$. The assertion (i) has been proved. (ii) Under the condition in (ii) ${ }^{t} \chi_{k, \nu}(\tau, \lambda) \zeta_{k, \nu^{\prime}}(\tau, \xi)$ takes the form $\sum_{n=0}^{\infty} c_{n} \sigma^{2 n+1}$ near $\sigma=0$. Changing the variable $\tau$ to $z=(1+i \operatorname{sh} \tau) / 2$, we deduce that the integral $\left\langle{ }^{t} \chi_{k, \nu} G_{\alpha} \zeta_{k, \nu^{\prime}}\right\rangle$ vanishes by Proposition 1.2 (i) [7] (cf. Proof of Lemma 2.4).

Q.E.D.

The next lemma is concerned with the if part of Theorem 2.2.

Lemma 2.8. Suppose that for any $k \leq k^{\prime}\left(\in Z_{+}+1 / 2\right)$

$$
\left\langle{ }^{t} \chi_{k, \nu}(\tau, \lambda) G_{\alpha} \zeta_{k, \nu^{\prime}}(\tau, \xi)\right\rangle=0, \quad(\lambda, \xi) \in R^{2}, \quad \operatorname{Re} \alpha>0, \quad \nu \nu^{\prime}<0 .
$$

(i) The integral vanishes even for $k=k^{\prime}+1$.

(ii) $D_{k, \pm}^{e}$ are invariant under the selfadjoint operators $L_{k}, M_{k}$ and the semigroup $T_{t}(t \geq 0)$. In particular, so are $D_{k^{\prime}+1, \pm}^{\ell}$ by (i).

Proof. It is clear that the linear span of $\left\{\zeta_{k, \nu} ; \pm \nu>0\right\}$ (resp. $\left\{\chi_{k, \nu}\right.$; $\pm \nu>0\}$ ) coincides with the one of $\left\{\psi_{k, \nu} ; \pm \nu>0\right\}$ (resp. $\left\{\tilde{\psi}_{k, \nu} ; \pm \nu>0\right\}$ ). We shall prove (ii) first. (ii) $D_{k, \pm}^{\ell}$ are invariant under $M_{k}$. Since

$$
L_{k} \Psi_{k, \nu}(\tau, \lambda)=\left\{\lambda^{2} /\left(4 \nu^{2}\right)-\nu^{2}\right\} \Psi_{k, \nu}(\tau, \lambda)
$$

by virtue of $(2.1) \sim(2.3), D_{k, \pm}^{\ell}$ are invariant under $L_{k}$ too. Put $I_{\alpha, \lambda, \xi}^{\nu, \nu^{\prime}}=$ $\left\langle\tilde{\Psi}_{k, \nu}(\tau, \lambda) G_{\alpha} \Psi_{k, \nu^{\prime}}(\tau, \xi)\right\rangle$, which is diagonal by the hypothesis. Since $I_{\alpha, \lambda, \xi}^{\nu, \nu^{\prime}}$ is diagonal, $\hat{\rho}_{k, \nu}=\rho_{k, \nu}{ }^{t} X_{\nu}^{-1}$ is also diagonal (see the proof of Theorem 1.1 [7]). Consequently, for any $h \in C_{0}(R)^{1}$ we have

$$
\begin{aligned}
& { }^{t} S_{\nu, \pm}(\lambda)\left[\mathscr{F}_{k} G_{\alpha} \int \Psi_{k, \nu^{\prime}}(\tau, \xi) \rho_{k, \nu^{\prime}}(\xi) r_{\nu^{\prime}, \pm}(\xi) h(\xi) d \xi\right](\lambda) \\
& \quad=\int{ }^{t} v_{ \pm} I_{\alpha, \lambda, \xi}^{\nu, \nu^{\prime}} \hat{\rho}_{k, \nu^{\prime}}(\xi)\left(\begin{array}{rr}
0 & 1 \\
-1 & 0
\end{array}\right) v_{ \pm} h(\xi) \operatorname{det} X_{\nu^{\prime}}(\xi) d \xi=0,
\end{aligned}
$$


where we used the following relations;

$$
\begin{gathered}
{ }^{t} s_{\nu, \pm} \Psi_{k, \nu^{\prime}}^{*}={ }^{t} v_{ \pm} \tilde{\Psi}_{k, \nu}, \quad{ }^{t} v_{ \pm}=(1 \pm 1,1 \mp 1) \\
\Psi_{k, \nu} \rho_{k, \nu} r_{\nu, \pm}=\Psi_{k, \nu} \hat{\rho}_{k, \nu}\left(\begin{array}{rr}
0 & 1 \\
-1 & 0
\end{array}\right) v_{ \pm} \operatorname{det} X_{\nu} .
\end{gathered}
$$

Thus $G_{\alpha}$ sends a dense subspace $\mathscr{F}_{k}^{-1}\left\{\left(r_{\nu, \pm} h_{\nu}\right) \in L^{2}\left(R, \rho_{k}\right) ; h_{\nu} \in C_{0}(R)^{1}\right\}$ of $D_{k, \pm}^{\ell}$ into $D_{k, \pm}^{e}$, namely $D_{k, \pm}^{e}$ is invariant under $T_{t}(t \geq 0)$. (i) First we shall prove the following relations in the case $\nu \nu^{\prime}<0$.

$$
\begin{aligned}
& \left\langle{ }^{t} \chi_{k, \nu} G_{\alpha}^{\prime} \zeta_{k, \nu^{\prime}}^{\prime}\right\rangle=0, \\
& \left\langle{ }^{t} \chi_{k, \nu} A_{k} G_{\alpha}^{\prime} \zeta_{k, \nu^{\prime}}\right\rangle=0, \\
& \left\langle{ }^{t} \chi_{k, \nu} M_{k} A_{k} G_{\alpha}^{\prime} \zeta_{k, \nu^{\prime}}\right\rangle=0, \\
& \left\langle{ }^{t} \chi_{k, \nu} G_{\alpha}^{\prime}\left\{2 i A_{k}^{2} \operatorname{th} \tau+U_{k} / \operatorname{ch} \tau\right\} \zeta_{k, \nu^{\prime}}\right\rangle=0 .
\end{aligned}
$$

For this purpose denote by $A$ the infinitesimal operator of the semigroup $T_{t}(t \geq 0)$, that is, $A=i \operatorname{sh} \tau$, and note that $f_{\nu^{\prime}}=\int \zeta_{k, \nu^{\prime}}(\tau, \xi) h(\xi) d \xi \in D_{k, \operatorname{sign} \nu^{\prime}}^{\ell}$ for any $h \in C_{0}(R)^{1}$ on account of (2.31). Since $\bar{L}_{k} \chi_{k, \nu}=\sum_{\mu ; \mu \nu>0} \tilde{a}_{k, \mu} \tilde{\psi}_{k, \mu}$, the $T_{t}$-invariance of $D_{k, \pm}^{\ell}$ implies $\left\langle\left\{L_{k} \bar{\chi}_{k, \nu}\right\}^{*} G_{\alpha} f_{\nu^{\prime}}\right\rangle=0$. Integration by parts and the fact that $L_{k} \zeta_{k, \nu^{\prime}}=\sum_{\mu ; \mu \nu^{\prime}>0} a_{k, \mu} \psi_{k, \mu}$ yield $\left\langle{ }^{t} \chi_{k, \nu}\left(-G_{\alpha}^{\prime \prime}-2 G_{\alpha}^{\prime} \partial / \partial \tau\right) f_{\nu^{\prime}}\right\rangle$ $=0$. On the other hand, an equality $G_{\alpha}^{\prime \prime}=A G_{\alpha}^{2}-2\left(1-A^{2}\right) G_{\alpha}^{3}$, together with the $T_{t}$-invariance of $D_{k, \pm}^{e}$, yields $\left\langle{ }^{t} \chi_{k, \nu} G_{\alpha}^{\prime \prime} f_{\nu^{\prime}}\right\rangle=0$. Now letting $h$ converge to the Dirac measure supported at $\xi$ in the now proved equality $\left\langle{ }^{t} \chi_{k, \nu} G_{\alpha}^{\prime} f_{\nu^{\prime}}^{\prime}\right\rangle=0$, we obtain (2.32). We can safely change the order of integration and the limiting procedure by Lemma 2.2. Starting with $\left\langle\left(M_{k} \bar{\chi}_{k, \nu}\right) * G_{\alpha} f_{\nu^{\prime}}\right\rangle=0$, we can verify (2.33) similarly. For the proof of (2.34), it suffices to substitute $\left(M_{k} \bar{\chi}_{k, \nu}\right) *$ for ${ }^{t} \chi_{k, \nu}$ in (2.33) and integrate by parts. It follows from (2.33) and (2.34) that $\left\langle{ }^{t} \chi_{k, \nu}\left\{A_{k} G_{\alpha}^{\prime} M_{k}-M_{k} A_{k} G_{\alpha}^{\prime}\right\} \zeta_{k, \nu^{\prime}}\right\rangle=0$, where \{\} takes the form $2 i A_{k}^{2} G_{\alpha}^{\prime \prime}+G_{\alpha}^{\prime} U_{k} / \operatorname{ch} \tau$, since $A_{k} V_{k}-V_{k} A_{k}=U_{k}$. We must show that $A_{k}^{2} G_{\alpha}^{\prime \prime}$ can be replaced by $G_{\alpha}^{\prime}$ th $\tau=A G_{\alpha}^{2}$. To this end notice that $A_{k} G_{\alpha}^{\prime}$ leaves $D_{k, \pm}^{e}$ invariant in virtue of (2.33). Consequently, since $(\alpha-A)\left(A_{k} G_{\alpha}^{\prime}\right)^{2}=A_{k}^{2}\left(1-A_{\alpha}^{2}\right) G_{\alpha}^{3}$, it follows that $\left\langle{ }^{t} \chi_{k, \nu} A_{k}^{2}\left(1-A^{2}\right) G_{\alpha}^{3} f_{\nu^{\prime}}\right\rangle$ $=0$ for the above-mentioned $f_{\nu^{\prime}}$. The last equality yields $\left\langle{ }^{t} \chi_{k, \nu} A_{k}^{2},\left(1-A^{2}\right)\right.$ $\left.\times G_{\alpha}^{3} \zeta_{k, \nu^{\prime}}\right\rangle=0$. Since $G_{\alpha}^{\prime \prime}=A G_{\alpha}^{2}-2\left(1-A^{2}\right) G_{\alpha}^{3}$, we can replace $A_{k}^{2} G_{\alpha}^{\prime \prime}$ by $G_{\alpha}^{\prime}$ th $\tau$ in the above \{\} . (2.35) has been proved. Secondly, to complete the proof of (ii), we shall show

$$
\left\langle{ }^{t} \chi_{k+1, \nu}(\tau, \lambda) G_{\alpha} \zeta_{k+1, \nu^{\prime}}(\tau, \xi)\right\rangle=0, \quad \nu \nu^{\prime}<0 .
$$

In view of Lemma 2.7, we may assume $\alpha_{k+1, \nu}+\alpha_{k+1, \nu^{\prime}}<0$. Hence $\chi_{k+1, \nu}=$ 
$\bar{K}_{+, k} \chi_{k, \nu}$ and $\zeta_{k+1, \nu^{\prime}}=K_{+, k} \zeta_{k, \nu^{\prime}}$. Integrating by parts in the left side of (2.36), we arrive at

$$
\left\langle{ }^{t} \chi_{k+1, \nu} G_{\alpha} \zeta_{k+1, \nu^{\prime}}\right\rangle=\left\langle{ }^{t} \chi_{k, \nu} G_{\alpha}^{\prime} B_{k} K_{+, k} \zeta_{k, \nu^{\prime}}\right\rangle .
$$

Note that $G_{\alpha}^{\prime} B_{k} K_{+, k}$ is equal to

$$
\begin{aligned}
G_{\alpha}^{\prime} & \left\{2 i(k+1)^{2} d / d \tau+\left(A_{k} M_{k}\right)+\left(2 i A_{k}^{2} \text { th } \tau+U_{k} / \operatorname{ch} \tau\right)\right\} \\
& -2 i(k+1)^{3}(\alpha-A)^{-1} A .
\end{aligned}
$$

Now the right side of (2.37) vanishes on account of (2.32), (2.33) and (2.35), together with the $T_{t}$-invariance of $D_{k, \pm}^{\ell}$.

Q.E.D.

The next lemma is concerned with the only if part of Theorem 2.2.

LEMMA 2.9 .

(i) ${ }^{t} x_{k, \pm 1 / 2,0} z_{k, \pm 1 / 2,0} \neq 0$ (see (2.24)).

(ii) For any $(\lambda, \xi) \in R^{2}$ and $\nu, \nu^{\prime}\left(\nu \nu^{\prime}>0\right)$, there exists an $\alpha(\operatorname{Re} \alpha>0)$ such that $\left\langle{ }^{t} \tilde{\psi}_{k, \nu}(\tau, \lambda) G_{\alpha} \psi_{k, \nu^{\prime}}(\tau, \xi)\right\rangle \neq 0$.

Proof. Let us define $z_{k, \pm}$ and $x_{k, \pm}$ both in $M_{2 k+1,1}$ so that they are proportional to $z_{k, \pm 1 / 2,0}$ and $x_{k, \pm 1 / 2,0}$ respectively;

$$
\begin{aligned}
& z_{k, \pm}=x_{k, \pm}={ }^{t}(1, \mp 1) \text { for } k=1 / 2, \\
& z_{k+1, \pm}=\left\{2(-2 k-1 / 2 \pm i \eta) B_{k}-Y_{k}\right\} z_{k, \pm}, \\
& x_{k+1, \pm}=\left\{-2(-2 k-1 / 2 \pm i \eta) B_{k}-\bar{Y}_{k}\right\} x_{k, \pm} .
\end{aligned}
$$

Then (i) is an immediate consequence of the following recursion formula;

$$
{ }^{t} x_{k+1, \pm} z_{k+1, \pm}=-8(k+1)(2 k+1)( \pm i \eta-k)( \pm i \eta-k-1 / 2)^{t} x_{k, \pm} z_{k, \pm} \cdot
$$

To prove (2.39), by the very definition of $x_{k, \pm}$ and $z_{k, \pm}$ we have

$$
\begin{aligned}
& { }^{t} x_{k+1, \pm} z_{k+1, \pm}={ }^{t} x_{k, \pm}\left[-4 \beta_{ \pm}^{2}\left\{(k+1)^{2}-A_{k}^{2}\right\}+2 \beta_{ \pm}(2 k+3)\right. \\
& \left.\quad+U_{k}-V_{k}^{2}+4(k+1)^{2}+(k+1) A_{k}^{2}-4(k+1)^{2} \ell(\ell+1)\right] z_{k, \pm},
\end{aligned}
$$

where $\beta_{ \pm}=-2 k-1 / 2 \pm i \eta$. At this stage observe that $\psi_{k, \pm k}=\sum_{ \pm \nu>0}$ $a_{k, \nu} \zeta_{k, \nu}$ and $\tilde{\psi}_{k, \pm k}=\sum_{ \pm \nu>0} \tilde{a}_{k, \nu} \chi_{k, \nu}$ with $a_{k, \pm 1 / 2} \tilde{a}_{k, \pm 1 / 2} \neq 0$ in view of Lemma 2.5 and the definitions of $\psi_{k, \pm k}$ and $\tilde{\psi}_{k, \pm k}$. Consequently, the equations $\left\{L_{k}+k^{2}-\lambda^{2} /\left(4 k^{2}\right)\right\} \Psi_{k, k}=0$ (see $\left.(2.28)\right),\left(M_{k}-\lambda\right) \Psi_{k, k}=0$ and $\left(\bar{M}_{k}-\lambda\right) \tilde{\Psi}_{k, k}$ $=0$ imply

$$
\begin{aligned}
\{-(-k+1 / 2 \pm i \eta)(-k-1 / 2 \pm i \eta)+ & k(k+1)+\ell(\ell+1) \\
& \left.-2 A_{k}^{2}+U_{k}\right\} z_{k, \pm}=0
\end{aligned}
$$




$$
\begin{aligned}
& \left\{-2(-k+1 / 2 \pm i \eta) A_{k}-V_{k}\right\} z_{k, \pm}=0 \\
& \left\{2(-k+1 / 2 \pm i \eta) A_{k}-\bar{V}_{k}\right\} x_{k, \pm}=0
\end{aligned}
$$

respectively. Recalling that $U_{k}=A_{k} V_{k}-V_{k} A_{k}$, it follows from (2.42) and (2.43) that both ${ }^{t} x_{k, \pm} U_{k} z_{k, \pm}$ and ${ }^{t} x_{k, \pm} V_{k}^{2} z_{k, \pm}$ are proportional to ${ }^{t} x_{k, \pm} A_{k}^{2} z_{k, \pm}$. Now (2.41) yields

$$
{ }^{t} x_{k, \pm} A_{k}^{2} z_{k, \pm}=k(1 \pm 2 i \eta) /\{4(-k+1 \pm i \eta)\}^{t} x_{k, \pm} z_{k, \pm},
$$

which enables us to rewrite the right side of (2.40) as (2.39). (ii) It is not difficult to show, by Lemma 2.5 and the definition of $\psi_{k, \nu}$ and $\tilde{\psi}_{k, \nu}$, that $\psi_{k, \nu}=\sigma^{\alpha k,(\operatorname{sign} \nu) 1 / 2}\left(\sum_{n=0}^{\infty} e_{k, \nu, n} \sigma^{n}\right)$ with $e_{k, \nu, 0} \propto z_{k,(\operatorname{sign} \nu) 1 / 2,0}$ and that $\tilde{\psi}_{k, \nu}=$ $\sigma^{\alpha k,(\operatorname{sign} \nu) 1 / 2}\left(\sum_{n=0}^{\infty} \tilde{e}_{k, \nu, n} \sigma^{n}\right)$ with $\tilde{e}_{k, \nu, 0} \propto x_{k,(\operatorname{sign} \nu) 1 / 2,0} . \quad$ Assume $\nu \nu^{\prime}>0$. Then ${ }^{t} \tilde{\psi}_{k, \nu}(\tau, \lambda) \psi_{k, \nu^{\prime}}(\tau, \xi)$ takes the form $\sigma^{2 \alpha k,(\operatorname{sign} \nu) 1 / 2}\left(\sum_{n=0}^{\infty} c_{n} \sigma^{n}\right)$ near $\sigma=0$, where $c_{0} \neq 0$ by (i). On the other hand, if $\left\langle{ }^{t} \tilde{\psi}_{k, \nu} G_{\alpha} \psi_{k, \nu}\right\rangle$ vanishes identically in $\{\operatorname{Re} \alpha>0\}$, then ${ }^{t} \tilde{\psi}_{k, \nu}(\tau, \lambda) \psi_{k, \nu}(\tau, \xi) /\{z(1-z)\}^{1 / 2}$, as a function of $z=(1+$ $i \operatorname{sh} \tau) / 2$, is holomorphic in $\{\operatorname{Re} z<1\}$ by Proposition 1.2 (ii) [7], which is absurd in view of Lemma 1.1 [7]. Thus $\left\langle{ }^{t} \tilde{\psi}_{k, \nu} G_{\alpha} \psi_{k, \nu}\right\rangle$ can not vanish identically.

Q.E.D.

We return to the

Proof of Theorem 2.2. We devide the proof into six parts.

1) The sequence $\left\{D_{k, \pm}^{e}\right\}$ fulfils the conditions (Q.1) and (Q.2).

First, the condition (Q.1) is satisfied by Theorem 2.1 and Lemma 2.8. Secondly, we shall prove that $K_{+, k} D_{k, \pm}^{\ell} \subset D_{k+1, \pm}^{\ell}$. For this purpose it suffices to show that, for any $f=\int \Psi_{k, \nu} \rho_{k, \nu} r_{\nu, \pm} h d \lambda$ lying in $H_{2}(R)^{2 k+1}$, $\mathscr{F}_{k+1} K_{+, k} f=\left(g_{k+1, \nu^{\prime}}\right)\left(\nu^{\prime}=1 / 2, \cdots, k+1\right)$ with $g_{k+1, \nu^{\prime}}=\delta_{\nu \nu^{\prime}} n_{k+1, \nu}^{-1} r_{\nu, \pm} h$. Integration by parts yields the desired $g_{k+1, \nu^{\prime}}$ on account of the formula $K_{+, k}^{*} \Psi_{k+1, \nu^{\prime}}=\left(1-\delta_{k+1, \nu^{\prime}}\right) n_{k+1, \nu^{\prime}}^{-1} \Psi_{k, \nu^{\prime}}$ due to (2.21). Finally, the inclusion relation $K_{+, k-1}^{*} D_{k, \pm}^{\ell} \subset D_{k-1, \pm}^{\ell}$ can be shown in a similar manner.

2) $K_{+, k-1}^{*} L^{2}(R)^{2 k+1}$ is dense in $L^{2}(R)^{2 k-1}$.

This statement is an easy consequence of Proposition 2.6 and (2.21).

3) Let $D$ be a closed subspace of $L^{2}(R)^{2 k+3}$ such that it is invariant under $T_{t}$ or $T_{-t}(t \geq 0)$ and that $K_{+, k} L^{2}(R)^{2 k+1} \subset D$. Then $D=L^{2}(R)^{2 k+3}$.

To be definite, let $T_{t}(t \geq 0)$ leave $D$ invariant. Assuming $f=\left(f_{\nu}\right) \in D^{\perp}$, we shall show $f=0$. We have, for any $\phi \in C_{0}^{\infty}(R)^{2 k+1}$,

$$
\left\langle f, K_{+, k} \phi\right\rangle=0, \quad\left\langle f, G_{\alpha} K_{+, k} \phi\right\rangle=0 .
$$

The first equality implies that $f_{\nu}(|\nu| \leq k)$ is absolutely continuous. Inte- 
grating by parts in the second equality and using the first one, we obtain $\left\langle B_{k} f, G_{\alpha}^{\prime} \phi\right\rangle=0$. Therefore $f_{\nu}(|\nu| \leq k)$ vanishes. Now the first equality yields $f=0$.

4) If $D_{k^{\prime}}=L^{2}(R)^{2 k^{\prime}+1}$ for some $k^{\prime}$, then $D_{k}=L^{2}(R)^{2 k+1}$ for all $k$.

Indeed, $D_{k}=L^{2}(R)^{2 k+1}$ for any $k>k^{\prime}$ by 3 ) and for any $k<k^{\prime}$ by 2 ).

5) If $D_{k^{\prime}}=\{0\}$ for some $k^{\prime}$, then $D_{k}=\{0\}$ for all $k$.

To prove this, consider a sequence $\left\{D_{k}^{\perp}\right\}$, each member of which is surely invariant under the selfadjoint operators $L_{k}, M_{k}$ and the semigroup $T_{-t}(t \geq 0)$. This sequence satisfies the condition (Q.2) too. To verify this, it is enough to note that, $D_{k}$ and $D_{k}^{\perp}$ being $L_{k}$-invariant, $D_{k} \cap H_{2}(R)^{2 k+1}$ and $D_{k}^{\perp} \cap H_{2}(R)^{2 k+1}$ are dense in $D_{k}$ and $D_{k}^{\perp}$ respectively. Applying 2) and 3) to $\left\{D_{k}^{\perp}\right\}$, we conclude that $D_{k}=\{0\}$.

6) Let $\left\{D_{k}\right\}$ be a nontrivial sequence satisfying the conditions (Q.1) and (Q.2). Then $\left\{D_{k}\right\}=\left\{D_{k,-}^{\ell}\right\}$ or $\left\{D_{k,+}^{\ell}\right\}$.

To begin with, note that $D_{k}$ is a proper subspace of $L^{2}(R)^{2 k+1}$ for any $k$ by 4 ) and 5). In particular $D_{k}=D_{k,-}^{e}$ or $D_{k,+}^{e}$ for $k=1 / 2$ on account of Theorem 2.1. Assuming that the latter is the case for definiteness, we shall show that $D_{k}=D_{k,+}^{e}$ for all $k$. To this end denote by $I$ the identity operator on $L^{2}(R)^{2 k+1}$, and by $P_{k, \nu}(\nu=1 / 2, \cdots, k)$ the orthogonal projection: $L^{2}(R)^{2 k+1} \rightarrow\left\{\int \Psi_{k, \nu} \rho_{k, \nu} g d \lambda ; g \in L^{2}\left(R, \rho_{k, \nu}\right)\right\}$. Suppose $D_{k^{\prime}}=D_{k^{\prime},+}^{\ell}$ for any $k^{\prime}<k$ $(k>1 / 2)$. As one can see easily, $K_{+, k-1} D_{k-1,+}^{\ell}$ is dense in $\left(I-P_{k, k}\right) D_{k,+}^{\ell}$, in particular $\left(I-P_{k, k}\right) D_{k,+}^{\ell} \subset D_{k}$ by the condition (Q.2). We claim that $D_{k,+}^{e} \subset D_{k}$. For this proof, recall Lemmas 2.8 and 2.9 , by which we have

$$
\begin{array}{ll}
\left\langle{ }^{t} \tilde{\psi}_{k,-k}(\tau, \lambda) G_{\alpha} \psi_{k, 1 / 2}(\tau, \xi)\right\rangle=0 & \text { for any } \alpha(\operatorname{Re} \alpha>0), \\
\left\langle{ }^{t} \tilde{\psi}_{k, k}(\tau, \lambda) G_{\alpha^{\prime}} \psi_{k, 1 / 2}(\tau, \xi)\right\rangle \neq 0 & \text { for some } \alpha^{\prime}\left(\operatorname{Re} \alpha^{\prime}>0\right) .
\end{array}
$$

Consequently there are an $\alpha^{\prime}$ in $\{\operatorname{Re} \alpha>0\}$ and an $f$ in $P_{k, 1 / 2} D_{k,+}^{e}$ such that $G_{\alpha^{\prime}} f \in D_{k,+}^{e}$ and $P_{k, k} G_{\alpha^{\prime}} f \neq 0$. For example, $f(\tau)=\int \Psi_{k, 1 / 2}(\tau, \xi) h(\xi) d \xi$ with $h \in C_{0}(R)^{1}$ such that $\left\langle{ }^{t} \tilde{\psi}_{k, k}(\tau, \lambda) G_{\alpha^{\prime}} \int \psi_{k, 1 / 2}(\tau, \xi) h(\xi) d \xi\right\rangle \neq 0$. The support of $\mathscr{F}_{k} P_{k, k} G_{\alpha^{\prime}} f$ is $R$, because $\mathscr{F}_{k} P_{k, k} G_{\alpha^{\prime}} f$ is real analytic on $R$. Therefore it is clear that the closed linear span of $\left\{\left[\exp i t M_{k}\right] P_{k, k} G_{\alpha^{\prime}} f ; t \in R\right\}$ coincides with $P_{k, k} D_{k,+}^{\ell}$. Since $P_{k, k} G_{\alpha^{\prime}} f$ belong to $D_{k,+}^{\ell} \cap D_{k}$ and $\left(I-P_{k, k}\right) D_{k,+}^{\ell} \subset D_{k}$, we conclude that $D_{k,+}^{e} \subset D_{k}$. Next, assuming $h \in D_{k} \ominus\left(I-P_{k, k}\right) D_{k,+}^{e}$, we will show that $h \in P_{k, k} D_{k}$, in other words, $D_{k}=P_{k, k} D_{k} \oplus\left(I-P_{k, k}\right) D_{k,+}^{e}$. Since $D_{k} \ominus\left(I-P_{k, k}\right) D_{k,+}^{\ell}$ is $L_{k}$-invariant, we may assume $h \in H_{2}(R)^{2 k+1}$. Note that $P_{k, \nu} h$ lies in the domain of $L_{k}$, because $P_{k, \nu}$ commutes with $L_{k}$. 
Now $K_{+, k-1}^{*} P_{k, k} h=0$. Using this equality, we will show that $\left(I-P_{k, k}\right) h$ $=0$. In fact, for any $f$ in $D_{k-1,+}^{\ell} \cap H_{2}(R)^{2 k-1}$ we have

$$
0=\left\langle K_{+, k-1} f, h\right\rangle=\left\langle f, K_{+, k-1}^{*} h\right\rangle=\left\langle f, K_{+, k-1}^{*}\left(I-P_{k, k}\right) h\right\rangle .
$$

By the induction hypothesis there results $K_{+, k-1}^{*}\left(I-P_{k, k}\right) h=0$, which yields $\left(I-P_{k, k}\right) h=0$, since $\mathscr{F}_{k}\left(I-P_{k, k}\right) h=0$. Thus $D_{k}=P_{k, k} D_{k} \oplus(I-$ $\left.P_{k, k}\right) D_{k,+}^{e}$. Finally, we shall prove that $P_{k, k} D_{k}=P_{k, k} D_{k,+}^{e}$ by showing the following equality;

$$
{ }^{t} s_{k,+}(\lambda) \int \Psi_{k, k}^{*}(\tau, \lambda) G_{\alpha} h(\tau) d \tau=0 \text { for }(\lambda, \alpha) \in R \times\{\operatorname{Re} \alpha>0\},
$$

where $h$ stands for the same as above. Suppose the integral in (2.44) does not vanish for some $\left(\lambda^{\prime}, \alpha^{\prime}\right)$. Then, since the integral is a real analytic function of $\lambda$, it is not equal to zero a.e. on $R$ for the $\alpha^{\prime}$. In particular

$$
\operatorname{det}\left(r_{k,+}(\lambda), \int \Psi_{k, k}^{*}(\tau, \lambda) G_{\alpha^{\prime}} h(\tau) d \tau\right) \neq 0 \text { a.e. on } R \text {. }
$$

$P_{k, k} D_{k}$ being $M_{k}$-invariant, it follows that

$$
P_{k, k} D_{k}=\left\{\int \Psi_{k, k} \rho_{k, k} g d \lambda ; g \in L^{2}\left(R, \rho_{k, k}\right)\right\} .
$$

In view of Lemma 2.9 , for any $(\lambda, \xi) \in R^{2}$ and $\nu(<k-1)$ there exists an $\alpha(\operatorname{Re} \alpha>0)$ such that

$$
{ }^{t} s_{\nu,+}(\lambda)\left\langle\Psi_{k, \nu}^{*}(\tau, \lambda) G_{\alpha} \psi_{k,-k}(\tau, \xi)\right\rangle=\left\langle{ }^{t} \tilde{\psi}_{k,-\nu}(\tau, \lambda) G_{\alpha} \psi_{k,-k}(\tau, \xi)\right\rangle \neq 0 .
$$

Consequently there exists an $f \in P_{k, k} D_{k}\left(\subset D_{k}\right)$ such that $P_{k, \nu} G_{\alpha} f \notin P_{k, \nu} D_{k,+}^{e}$, which contradicts the fact $D_{k}=P_{k, k} D_{k} \oplus\left(I-P_{k, k}\right) D_{k,+}^{\ell}$. Thus (2.44) holds.

Q.E.D.

By Proposition 2.6 and (2.21), it is not hard to see that, for

$$
f=\sum_{\nu=1 / 2}^{k} \int \Psi_{k, \nu} \rho_{k, \nu} r_{\nu, \pm} h_{\nu} d \lambda, \quad h_{\nu} \in C_{0}(R)^{1}
$$

we have

$$
\begin{aligned}
K_{+, k-1}^{*} f & =\sum_{\nu=1 / 2}^{k-1} \int \Psi_{k-1, \nu} \rho_{k-1, \nu} r_{\nu, \pm} h_{\nu} d \lambda \\
K_{+, k} f & =\sum_{\nu=1 / 2}^{k} \int \Psi_{k+1, \nu} \rho_{k+1, \nu} r_{\nu, \pm} n_{k+1, \nu}^{-1} h_{\nu} d \lambda .
\end{aligned}
$$


§ 3. Invariant subspaces common to $L_{k, \ell}, M_{k, \ell}$ and $T_{t}(t \geq 0)$ in $L^{2}(R)^{2 k+1}, k \in Z_{+}$

The purpose of this section is to determine all nontrivial sequence $\left\{D_{k}\right\}_{k \in Z_{+}}$satisfying the conditions (Q.1) and (Q.2) (see the beginning of $\S 2$ ) in the case $(\ell, \varepsilon)=(\ell, 0)$ with either $\ell=-1 / 2+$ in or $-1<\ell<-1 / 2$. Throughout $\S 3$ it is assumed that $k \in Z_{+}$and $\ell=-1 / 2+i \eta(\eta \geq 0)$ or $-1<\ell<-1 / 2$. Our reasoning will follow almost the same line as in $\S 2$, except that the eigenfunction expansion for $L_{k}$ as well as for $M_{k}$ will be used. This is because in the orthogonal decomposition

$$
L^{2}(R)^{2 k+1}=\operatorname{Ker} M_{k} \oplus\left(\operatorname{Ker} M_{k}\right)^{\perp}
$$

$\operatorname{Ker} M_{k}$ is infinite dimensional, for it contains $K_{+, k-1} \cdots K_{k, 0} C_{0}^{\infty}(R)^{1}$.

Let $\tilde{\theta}_{k}(\tau, \lambda) \in M_{2 k+1,4 k+2}$ and $\Phi_{k}(\tau, \lambda) \in M_{2 k+1,2 k}$ be solutions of the following equations respectively;

$$
\begin{aligned}
\left(L_{k}-\lambda\right) \tilde{\theta}_{k}=0, & { }^{t}\left({ }^{t} \tilde{g}_{k},{ }^{t} \tilde{\theta}_{k}^{\prime}\right)_{\tau=0} & =I_{4 k+2}, \\
\left(M_{k}-\lambda\right) \Phi_{k}=0, & \check{\Phi}_{k}(0, \lambda) & =I_{2 k},
\end{aligned}
$$

where $\check{\Phi}_{k}(\tau, \lambda)$ denotes the matrix obtained by deleting the 0 -th row of $\Phi_{k}(\tau, \lambda)$ (the 0 -th row of $A_{k}$ is equal to 0 ). Since $L_{k}+d^{2} / d \tau^{2}+A_{k}^{2}$ is a multiplication by an integrable function, the spectral matrix $\tilde{\Sigma}_{k}$ for $L_{k}$ relative to the generalized eigenfunction $\tilde{\theta}_{k}$ has a spectral density $\tilde{\sigma}_{k}$ on $R_{+}$[5, Theorem 15] which fulfils the following conditions i) iii) (cf. [2, p. 264]).

i) $\tilde{\sigma}_{k}$ is an $M_{4 k+1}^{++}$-valued continuous function on $R_{+}$.

ii) A map $\widetilde{\mathscr{E}}_{k}: L^{2}(R)^{2 k+1} \rightarrow L^{2}\left(R_{+}, \tilde{\sigma}_{k}\right)$ defined by

$$
\widetilde{\mathscr{E}}_{k} f(\lambda)=\underset{N \rightarrow \infty}{\operatorname{lij} . \mathrm{m} .} \int_{|\tau|<N} \tilde{\theta}_{k}^{*}(\tau, \lambda) f(\tau) d \tau
$$

is an onto partial isometry, whose inverse $\widetilde{\mathscr{E}}_{k}^{-1}: L^{2}\left(R_{+}, \tilde{\sigma}_{k}\right) \rightarrow L^{2}(R)^{2 k+1}$ is given by

$$
\widetilde{E}_{k}^{-1} g(\tau)=\lim _{N \rightarrow \infty} \int_{0<\lambda<N} \tilde{\theta}_{k}(\tau, \lambda) \tilde{\sigma}_{k}(\lambda) g(\lambda) d \lambda .
$$

iii) $\widetilde{\mathscr{E}}_{k} L_{k} \widetilde{\mathscr{E}}_{k}^{-1} g(\lambda)=\lambda g(\lambda)$ if $\lambda g(\lambda)$ belongs to $L^{2}\left(R_{+}, \tilde{\sigma}_{k}\right)$.

As to the eigenfunction expansion for $M_{k}$, there exists a spectral density $\tilde{\rho}_{k}$ on $R^{*}$ satisfying the following conditions iv) $\sim$ vi) [5, Theorem 14].

iv) $\tilde{\rho}_{k}$ is a $M_{2 k}^{++}$-valued continuous function on $R^{*}$. 
v) A map $\tilde{\mathscr{F}}_{k}: L^{2}(R)^{2 k+1} \rightarrow L^{2}\left(R^{*}, \tilde{\rho}_{k}\right)$ defined by

$$
\tilde{\mathscr{F}}_{k} f(\lambda)=\operatorname{lin}_{N \rightarrow \infty} \int_{|\tau|<N} \Phi_{k}^{*}(\tau, \lambda) f(\tau) d \tau
$$

is an onto isometry with $\operatorname{Ker} \tilde{\mathscr{F}}_{k}=\operatorname{Ker} M_{k}$. The inverse $\tilde{\mathscr{F}}_{k}^{-1}$ is given by

$$
\tilde{\mathscr{F}}_{k}^{-1} g(\tau)=\underset{N \rightarrow \infty}{\operatorname{li} . \mathrm{m}} \int_{N-1<|\lambda|<N} \Phi_{k}(\tau, \lambda) \tilde{\rho}_{k}(\lambda) g(\lambda) d \lambda \text {. }
$$

vi) $\tilde{\mathscr{F}}_{k} M_{k} \tilde{\mathscr{F}}_{k}^{-1} g(\lambda)=\lambda g(\lambda)$ if $\lambda g(\lambda)$ lies in $L^{2}\left(R^{*}, \tilde{\rho}_{k}\right)$.

All closed, proper invariant subspaces common to $L_{0}$ and $T_{t}(t \geq 0)$ is known [7, Theorem 1.1]. To define these subspaces again, denote by $\left\{\zeta_{0, \nu}(\tau, \lambda) ;|\nu|=1 / 4\right\}$ a fundamental system of an equation $\left(L_{0}-\lambda\right) \zeta=0$, each member of which, being holomorphic in $\dot{D}_{\tau} \times C$, is assumed to have the following form near $\sigma=0$;

$$
\left.\begin{array}{ll}
\zeta_{0, \nu}=\sigma^{\alpha_{0, \nu}}\left(\sum_{n=0}^{\infty} z_{0, \nu, n} \sigma^{n}\right) & \text { if } \ell \neq-1 / 2, \\
\zeta_{0,1 / 4}=\sigma^{1 / 2}\left(\sum_{n=0}^{\infty} z_{0,1 / 4, n} \sigma^{n}\right) & \\
\zeta_{0,-1 / 4}=\zeta_{0,1 / 4} \log \sigma+\sigma^{1 / 2}\left(\sum_{n=1}^{\infty} z_{0,-1 / 4, n} \sigma^{n}\right)
\end{array}\right\} \quad \text { if } \ell=-1 / 2 .
$$

In the above $\alpha_{0, \pm 1 / 4}=1 / 2 \pm(\ell+1 / 2)$ and $z_{0, \nu, 0}=1$. Put $\zeta_{0}=\left(\zeta_{0,-1 / 4}, \zeta_{0,1 / 4}\right)$, and define $X_{0}(\lambda) \in M_{2}$ and $s_{0, \pm}(\lambda), r_{0, \pm}(\lambda) \in M_{2,1}$ by

$$
\zeta_{0}(\tau, \lambda)=\tilde{\theta}_{0}(\tau, \lambda) X_{0}(\lambda), \quad s_{0, \pm}=X_{0} v_{ \pm}, \quad r_{0, \pm}=\left(\begin{array}{rr}
0 & 1 \\
-0 & 1
\end{array}\right) s_{0, \pm}
$$

where $v_{ \pm}={ }^{t}(1 \pm 1,1 \mp 1)$ or ${ }^{t}(0,2)$ according as $\ell \neq-1 / 2$ or not. Now we define closed proper subspaces $D_{0, \pm}^{\ell}$ of $L^{2}(R)^{1}$ by

$$
D_{0, \pm}^{\ell}=\widetilde{\mathscr{O}}_{0}^{-1}\left\{g \in L^{2}\left(R_{+}, \tilde{\sigma}_{0}\right) ;{ }^{t} s_{0, \pm} g(\lambda)=0 \text { a.e. }\right\} .
$$

Proposition 3.1. Let $D_{0}$ be a closed proper subspace of $L^{2}(R)$. Then $D_{0}$ is invariant under the selfadjoint operator $L_{0}$ and the semigroup $T_{t}(t \geq 0)$ iff $D_{0}$ coincides with one of $D_{0, \pm}^{\ell}$.

Let us introduce a fundamental matrix $\theta_{k}=\left(\theta_{k, 0}, \theta_{k, 1}, \cdots, \theta_{k, k}\right)$ of the equation $\left(L_{k}-\lambda\right) \theta=0$ in order to reduce the spectral density $\tilde{\sigma}_{k}$ to a simpler one;

$$
\theta_{0}=\zeta_{0}, \quad \theta_{k, \nu}=K_{+, k-1} \theta_{k-1, \nu}(0 \leq \nu<k) \text { or } \theta_{k, k}(\nu=k),
$$


where $\theta_{k, k}(\tau, \lambda)$, being holomorphic in $\dot{D}_{\tau} \times C$, satisfies $K_{+, k-1}^{*} \theta_{k, k}=0$ too (notice that the $k$-th row of $\theta_{k, k}(k \geq 1)$ is a solution of a 4 -th order differential equation). Set

$$
\tilde{\theta}_{k}(\tau, \lambda)=\theta_{k}(\tau, \lambda) Y_{k}(\lambda), \quad \sigma_{k}=Y_{k} \tilde{\sigma}_{k} Y_{k}^{*} .
$$

Then $\widetilde{\mathscr{E}}_{k}$ and $\widetilde{\mathscr{E}}_{k}^{-1}$ give rise to an onto partial isometry $\mathscr{E}_{k}: L^{2}(R)^{2 k+1} \rightarrow$ $L^{2}\left(R_{+}, \sigma_{k}\right)$ and its inverse $\mathscr{E}_{k}^{-1}: L^{2}\left(R_{+}, \sigma_{k}\right) \rightarrow L^{2}(R)^{2 k+1}$;

$$
\begin{aligned}
\mathscr{E}_{k} f(\lambda) & =\underset{N \rightarrow \infty}{\lim .} \int_{|\tau|<N} \theta_{k}^{*}(\tau, \lambda) f(\tau) d \tau, \\
\mathscr{E}_{k}^{-1} g(\tau) & =\underset{N \rightarrow \infty}{\lim . \operatorname{m}} \int_{0<\lambda<N} \theta_{k}(\tau, \lambda) \sigma_{k}(\lambda) g(\lambda) d \lambda .
\end{aligned}
$$

The eigenfunction expansion for $L_{k}$ relative to $\theta_{k}$ has an advantage, because we have

Proposition 3.2.

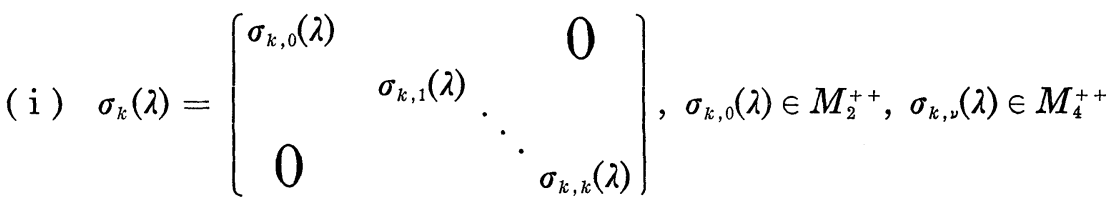

(ii) $\sigma_{k, \nu}=m_{k, \nu} \sigma_{k-1, \nu}$, where $m_{k, \nu}(\lambda)=\left\{4\left(k^{2}-\nu^{2}\right)\left(k^{2}+\nu^{2}+\lambda\right)\right\}^{-1}$

Proof. The proof follows the same development of that of Proposition 2.6, using Lemma 3.3 and an equality

$$
K_{+, k-1}^{*} K_{+, k-1} \theta_{k-1, \nu}(\tau, \lambda)=m_{k, \nu}^{-1}(\lambda) \theta_{k-1, \nu}(\tau, \lambda),
$$

which is an easy consequence of the fundamental relations $(2.1) \sim(2.3)$.

Q.E.D.

LEMMA 3.3.

(i) Fix positives $\lambda_{0}$ and $\varepsilon$. Then there are positives $\delta$ and $K$ such that

$$
\begin{array}{ll}
\left|\tilde{\theta}_{k}\left(\tau, \lambda_{0}\right)\right|+\left|\tilde{\theta}_{k}^{\prime}\left(\tau, \lambda_{0}\right)\right|<K & \text { on } \bar{D}_{\tau} \cap\{|\operatorname{Re} \tau| \geq 1\}, \\
\left|\tilde{\theta}_{k}(\tau, \lambda)\right|+\left|\tilde{\theta}_{k}^{\prime}(\tau, \lambda)\right|<K e^{\varepsilon|\tau|} & \text { on } R \times\left\{\left|\lambda-\lambda_{0}\right|<\delta\right\} .
\end{array}
$$

(ii) Assume $\hat{\theta}_{k}(\tau, \lambda) \in M_{2 k+1,4 k+2}$ satisfies the equation $\left(\bar{L}_{k}-\lambda\right) \hat{\theta}_{k}=0$ with initial value ${ }^{t}\left(\hat{\theta}_{k},{ }^{t} \hat{\theta}_{k}^{\prime}\right)_{\tau=0}=I_{4 k+2}$. Then the statement (i) holds for $\hat{\theta}_{k}$.

Proof. Put $\tilde{\theta}_{k}={ }^{t}\left({ }^{t} \tilde{\theta}_{k},{ }^{t} \tilde{\theta}_{k}^{\prime}\right)$ and $V(\tau)=\{2-k(k+1)-\ell(\ell+1)\} / \operatorname{ch}^{2} \tau+$ $i U_{k} \operatorname{th} / \mathrm{ch} \tau$. Then 


$$
\tilde{\theta}_{k}^{\prime}=\left(\begin{array}{cc}
0 & I_{2 k+1} \\
-A_{k}^{2}+V-\lambda & 0
\end{array}\right) \tilde{\theta}_{k}, \quad \tilde{\theta}_{k}(0, \lambda)=I_{4 k+2} .
$$

As is known ( $[5, \S 3]$, for example), there are $M_{4 k+2}$-valued continuous functions $T_{ \pm}(\lambda)$ on $\{\operatorname{Re} \lambda>0, \pm \operatorname{Im} \lambda \geq 0\}$ such that

$$
T_{ \pm}(\lambda)\left(\begin{array}{cc}
0 & I_{2 k+1} \\
-A_{k}^{2}+V-\lambda & 0
\end{array}\right) T_{ \pm}^{-1}(\lambda)=\left(\begin{array}{ccc}
\sqrt{-\lambda} J_{1} & & 0 \\
& \sqrt{-1-\lambda} J_{2} & \\
0 & & \ddots \sqrt{-k^{2}-\lambda} J_{2}
\end{array}\right),
$$

where $J_{n}(n \geq 1)$ means the diagonal matrix $\left(\begin{array}{cc}I_{n} & 0 \\ 0 & -I_{n}\end{array}\right) \in M_{2 n}$. Denote by $B_{ \pm}(\lambda)$ the above matrix, and put $\theta_{k, \pm}=T_{ \pm} \tilde{\theta}_{k}$. Then $\theta_{k, \pm}$ satisfies an equation $\theta_{k, \pm}^{\prime}=\left(B_{ \pm}+R_{ \pm}\right) \theta_{k, \pm}$ for $R_{ \pm}=T_{ \pm}\left(\begin{array}{cc}0 & 0 \\ V & 0\end{array}\right) T_{ \pm}^{-1}$. From now on we can argue as in the proof of Lemma 2.2 .

The subspace $\operatorname{Ker} M_{k}$ in $L^{2}(R)^{2 k+1}$ can be identified with $L^{2}\left(R_{+}, \sigma_{k, 0}\right)$ in a sense.

LEMMA 3.4. $\operatorname{Ker} M_{k}=\mathscr{E}_{k}^{-1} L^{2}\left(R_{+}, \sigma_{k, 0}\right)$, where $L^{2}\left(R_{+}, \sigma_{k, 0}\right)$ is regarded as a subspace of $L^{2}\left(R_{+}, \sigma_{k}\right)$.

Proof. First we claim that $L_{k}$ has no eigenvalues. To prove this assertion by induction, assume it to be true up to $k-1(k>0)$, and let $\left(L_{k}-\lambda\right) f=0$ for some $f \in H_{2}(R)^{2 k+1} \backslash\{0\}$. Then $\left(L_{k-1}-\lambda\right) K_{+, k-1}^{*} f=0$, which implies, by the induction hypothesis, that $K_{+, k-1}^{*} f=0$. Thanks to (2.3), $M_{k}^{2} f=4 k^{2}\left(k^{2}+\lambda\right) f$. If $\lambda \neq-k^{2}$, then $f \in\left(\operatorname{Ker} M_{k}\right)^{\perp}$, hence, rewriting the equality in $L^{2}\left(R^{*}, \tilde{\rho}_{k}\right), f=0$. This is absurd. On the other hand, if $\lambda=$ - $k^{2}$, then $M_{k}^{2} f=0$, which means $f \in \operatorname{Ker} M_{k}$, since $M_{k} f \in\left(\operatorname{Ker} M_{k}\right)^{\perp} \cap$ Ker $M_{k}=\{0\}$. As will be shown later (Lemma 3.13), an $f$ lying in $H_{2}(R)^{2 k+1}$ satisfies a condition that $K_{+, k-1}^{*} f=M_{k} f=0$ iff $f=0$. Thus $L_{k}$ has no eigenvalues. Secondly, we shall show that $\operatorname{Ker} M_{k} \subset \mathscr{E}_{k}^{-1} L^{2}\left(R_{+}, \sigma_{k, 0}\right)$ to conclude the proof, for the opposite inclusion is trivial on account of the relation $M_{k} \theta_{k, 0}=0$, which is due to (2.1). Suppose the inclusion relation holds up to $k-1(k>0)$. Then it follows from (2.1), (2.3) and Proposition 3.2 that, for any $f \in H_{2}(R)^{2 k+1} \cap \operatorname{Ker} M_{k},\left(4 k^{4}+4 k^{2} L_{k}\right) f$ lies in $\mathscr{E}_{k}^{-1} L^{2}\left(R_{+}, \sigma_{k, 0}\right)$, in particular in $\mathscr{E}_{k}^{-1} L^{2}\left(R_{+}, \sigma_{k}\right)$. Since $L_{k}$ has no eigenvalues, there results $f \in \mathscr{E}_{k}^{-1} L^{2}\left(R_{+}, \sigma_{k}\right)$. Now using $M_{k}^{2} \theta_{k, \nu}=-4 \nu^{2}\left(\nu^{2}+\lambda\right) \theta_{k, \nu}$, it can be easily shown that $f \in \mathscr{E}_{k}^{-1} L^{2}\left(R_{+}, \sigma_{k, 0}\right)$. Since $H_{2}(R)^{2 k+1} \cap \operatorname{Ker} M_{k}$ is dense in the $L_{k}$-invariant subspace $\operatorname{Ker} M_{k}$, the desired inclusion relation holds for $k$.

Q.E.D. 
For our later use, set $(\nu= \pm 1 / 4)$

$$
\begin{aligned}
& \alpha_{k, \nu}=-k+1 / 2+(\operatorname{sign} \nu)(\ell+1 / 2), \\
& \zeta_{k, \nu}=\psi_{k, \nu}=K_{+, k-1} \cdots K_{+, 0} \zeta_{0, \nu}, \quad \Psi_{k, 0}=\left(\psi_{k,-1 / 4}, \psi_{k, 1 / 4}\right), \\
& \chi_{k, \nu}=\tilde{\psi}_{k, \nu}=\bar{K}_{+, k-1} \cdots \bar{K}_{+, 0} \zeta_{0, \nu}, \quad \tilde{\Psi}_{k, 0}=\left(\tilde{\psi}_{k,-1 / 4}, \tilde{\psi}_{k, 1 / 4}\right), \\
& \rho_{k, 0}=\sigma_{k, 0} \text { on } R_{+} \text {while } \rho_{k, 0}=0 \text { on } R \backslash R_{+}, \\
& n_{k, 0}=m_{k, 0} \text { on } R_{+} \text {while } n_{k, 0}=0 \text { on } R \backslash R_{+} .
\end{aligned}
$$

Next, we intend to reduce the spectral density $\tilde{\rho}_{k}$ for $M_{k}$ to a simpler one by the aid of another generalized eigenfunction $\Psi_{1, k}$. For this purpose some preliminary considerations are necessary. Let $\zeta={ }^{t}\left(\zeta_{k}, \cdots, \zeta_{-k}\right)$ and $\chi={ }^{t}\left(\chi_{k}, \cdots, \chi_{-k}\right)$ be solutions of the following equations $(\lambda \neq 0)$;

$$
\left(M_{k}-\lambda\right) \zeta=0, \quad \overline{(3.15)} \quad\left(\bar{M}_{k}-\lambda\right) \chi=0 .
$$

Then $\zeta_{\nu}\left(\right.$ resp. $\chi_{\nu}$ ) can be represented in terms of $\zeta_{k}$ (resp. $\chi_{k}$ ) and its derivatives;

$$
\begin{aligned}
& \zeta_{\nu}(\tau, \lambda)=\sum_{m=0}^{k-\nu} \sum_{n=0}^{k-\nu-m} a_{k, \nu, m, n}(\tau) \zeta_{k}^{(m)}(\tau) \lambda^{n}, \\
& \chi_{\nu}(\tau, \lambda)=\sum_{m=0}^{k-\nu} \sum_{n=0}^{k-\nu-m} \tilde{a}_{k, \nu, m, n}(\tau) \chi_{k}^{(m)}(\tau) \lambda^{n} .
\end{aligned}
$$

Furthermore, it is not hard to verify that $\zeta_{k}$ and $\chi_{k}$ satisfy $2 k$-th order differential equations of the following form respectively;

$$
\begin{array}{ll}
\sum_{n=0}^{2 k} \sigma^{n} b_{k, 2 k-n}(\sigma, \lambda) \zeta_{k}^{(n)}=0, & b_{k, 0}=1, \\
\sum_{k=0}^{2 k} \sigma^{n} \tilde{b}_{k, 2 k-n}(\sigma, \lambda) \chi_{k}^{(n)}=0, & \tilde{b}_{k, 0}=1,
\end{array}
$$

where $b_{k, n}$ and $\tilde{b}_{k, n}$ are holomorphic in $\dot{D}_{\tau} \times C$ and $b_{k, n}(0, \lambda)$ and $\tilde{b}_{k, n}(0, \lambda)$ are independent of $\lambda$. Conversely, if $\zeta_{k}$ and $\chi_{k}$ solve (3.17) and (3.17) respectively, then $\zeta$ and $\chi$ defined by (3.16) and (3.16) satisfy (3.15) and $\overline{(3.15)}$ respectively.

LEMMA 3.5. The set of indicial roots of the equation (3.17) at $\sigma=0$ is $\left\{\alpha_{k, \nu} ; \nu= \pm 1, \pm 2, \cdots, k\right\}$, where

$$
\alpha_{k, \nu}=-k-3 / 2+(\operatorname{sign} \nu)(\ell+1 / 2+2 \nu) .
$$

The same is true for the set of indicial roots of (3.17).

For the proof we require the following lemma. 
LEMMA 3.6. Let $\zeta$ and $\chi$ be solutions of (3.15) and (3.15) respectively. Then $\zeta$ and $\chi$ solve

$$
K_{+, k-1}^{*} \zeta=0, \quad \overline{(3.18)} \quad \bar{K}_{+, k-1}^{*} \chi=0,
$$

respectively, iff $\zeta_{k}$ and $\chi_{k}$ satisfy certain differential equations of the form

$$
\begin{array}{ll}
\sum_{n=0}^{2} \sigma^{n} c_{k, 2-n}(\sigma, \lambda) \zeta_{k}^{(n)}=0, & c_{k, 0}=1 . \\
\sum_{n=0}^{2} \sigma^{n} \tilde{c}_{k, 2-n}(\sigma, \lambda) \chi_{k}^{(n)}=0, & \tilde{c}_{k, 0}=1,
\end{array}
$$

where $c_{k, n}$ and $\tilde{c}_{k, n}$, being holomorphic in $\dot{D}_{\tau} \times C$, satisfy the following condition.

$$
\begin{aligned}
\alpha(\alpha-1)+c_{k, 1}(0, \lambda) \alpha+c_{k, 2}(0, \lambda) & =\left(\alpha-\alpha_{k,-1}\right)\left(\alpha-\alpha_{k, 1}\right) \\
& =\alpha(\alpha-1)+\tilde{c}_{k, 1}(0, \lambda) \alpha+\tilde{c}_{k, 2}(0, \lambda) .
\end{aligned}
$$

Proof. Even though some involved calculation is needed, the proof of the only if part is straightforward. To prove the if part, note that when $K_{+, k-1}^{*}$ is regarded as a linear map sending solutions of (3.15) into those of (3.15) with suffix $k-1, \operatorname{dim}\left(\operatorname{Ker} K_{+, k-1}^{*}\right) \geq 2$. The only if part means $\operatorname{dim}\left(\operatorname{Ker} K_{+, k-1}^{*}\right) \leq 2$. Now that $\operatorname{dim}\left(\operatorname{Ker} K_{+, k-1}^{*}\right)=2$, any solution $\zeta_{k}$ of (3.19) gives a solution $\zeta$ of (3.18) defined by the formula (3.16).

Q.E.D.

Proof of Lemma 3.5. It suffices to prove the lemma in the case $\ell \neq$ $-1 / 2$, for $b_{k, n}(0, \lambda)$ and $\tilde{b}_{k, n}(0, \lambda)$ are continuous in $\ell$. We will treat only (3.17). When $k=1$, the lemma holds by Lemma 3.6. Assume it to be valid up to $k-1(k>1)$. Then (3.15) has solutions $\left\{\zeta_{k, \nu} ; 1 \leq|\nu| \leq k-1\right\}$ such that

$$
\left(\zeta_{k, \nu}\right)_{k}=\sigma^{\alpha k, \nu}\left(\sum_{n=0}^{\infty} z_{k, \nu, n, k} \sigma^{n}\right), \quad z_{k, \nu, 0, k} \neq 0,
$$

where $\left(\zeta_{k, \nu}\right)_{k}$ denotes the $k$-th row of $\zeta_{k, \nu}$. One can verify this statement inductively, using Lemma A.1 [7] and (2.1). By Lemma A.1 $\alpha_{k, \nu}(1 \leq|\nu|$ $\leq k-1)$ is an indicial root of the equation (3.17). We claim that all indicial roots of (3.17) are simple. Otherwise, denote by $\alpha$ a multiple root. Then by Lemma A.1 there exists a solution $\zeta$ of (3.15) such that $(\zeta)_{k}=\sigma^{\alpha}(\log \sigma+\sigma h(\sigma, \log \sigma))$. In particular $\left(K_{+, k-1}^{*} \zeta\right)_{k-1}=\sigma^{\alpha-1}(a \log \sigma+b$ $+\sigma h(\sigma, \log \sigma))$, where $a \propto\left(\alpha-\alpha_{k,-1}\right)\left(\alpha-\alpha_{k, 1}\right)$ and $b \propto \alpha+k+1 / 2$. Since no solution of (3.17) with suffix $k-1$ contains the logarithmic term, it 
follows that $\alpha=\alpha_{k, \pm 1}$. In this case, since $b \neq 0$, one of $\alpha_{k, \pm 1}-1$ is an indicial root of (3.17) by Lemma A.1, which contradicts the induction hypothesis. Now denote by $\alpha$ the one of unknown roots. Again by Lemma A.1 there exists a solution $\zeta$ of (3.15) such that $(\zeta)_{k}=\sigma^{\alpha}(1+\sigma h(\sigma, \log \sigma))$. In particular $\left(K_{+, k-1}^{*} \zeta\right)_{k-1}=\sigma^{\alpha-1}(a+\sigma h(\sigma, \log \sigma))$. Since $a \neq 0$, it follows that $\alpha$ belongs to $\left\{1+\alpha_{k-1, \nu} ; 1 \leq|\nu| \leq k-1\right\} \mid\left\{\alpha_{k, \nu} ; 1 \leq|\nu| \leq k-1\right\}$, namely $\alpha=1+\alpha_{k-1, \pm(k-1)}=\alpha_{k, \pm k}$. Q.E.D.

Now we are in a position to define a fundamental system $\left\{\psi_{k, \nu} ; 1 \leq\right.$ $|\nu| \leq k\}$ of (3.15)

$$
\psi_{k, \nu}=K_{+, k-1} \psi_{k-1, \nu}(1 \leq|\nu|<k) \text { or } \psi_{k, \nu}(|\nu|=k),
$$

where the $k$-th row $\left(\psi_{k, \pm k}\right)_{k}$ satisfies (3.19) and is a holomorphic function on $\dot{D}_{\tau} \times C$ assuming the following form near $\sigma=0$;

$$
\begin{aligned}
& \left(\psi_{k, \pm k}\right)_{k}=\sigma^{\alpha k, \pm 1}\left(\sum_{n=0}^{\infty} e_{k, \pm k, n, k} \sigma^{n}\right), e_{k, \pm k, 0, k}=1 \text { if } \ell \neq-1 / 2, \\
& \left(\psi_{k, k}\right)_{k}=\sigma^{\alpha k, 1}\left(\sum_{n=0}^{\infty} e_{k, k, n, k} \sigma^{n}\right), e_{k, k, 0, k}=1 \\
& \left.\left(\psi_{k,-k}\right)_{k}=\left(\psi_{k, k}\right)_{k} \log \sigma+\sigma^{\alpha_{k}, 1}\left(\sum_{n=1}^{\infty} e_{k,-k, n, k} \sigma^{n}\right)\right\} \text { if } \ell=-1 / 2 \text {. }
\end{aligned}
$$

Put $\Psi_{k, \nu}=\left(\psi_{k,-\nu}, \psi_{k, \nu}\right)$ and $\Psi_{1, k}=\left(\Psi_{k, 1}, \cdots, \Psi_{k, k}\right)$, and define $Z_{k}(\lambda), \rho_{1, k}(\lambda) \in$ $M_{2 k}$ by

$$
\Phi_{k}(\tau, \lambda)=\Psi_{1, k}(\tau, \lambda) Z_{k}(\lambda), \quad \rho_{1, k}=Z_{k} \tilde{\rho}_{k} Z_{k}^{*}
$$

Then $\widetilde{F}_{k}$ and $\tilde{F}_{k}^{-1}$ give rise to an onto partial isometry $\mathscr{F}_{1, k}: L^{2}(R)^{2 k+1} \rightarrow$ $L^{2}\left(R^{*}, \rho_{1, k}\right)$ and its inverse $\mathscr{F}_{1, k}^{-1}: L^{2}\left(R^{*}, \rho_{1, k}\right) \rightarrow L^{2}(R)^{2 k+1}$;

$$
\begin{aligned}
& \mathscr{F}_{1, k} f(\lambda)=\operatorname{lij.m} . \int_{|\tau|<N} \Psi_{1, k}^{*}(\tau, \lambda) f(\tau) d \tau, \\
& \mathscr{F}_{1, k}^{-1} g(\tau)=\operatorname{1.i.m} . \int_{N \rightarrow \infty} \Psi_{1, k}(\tau, \lambda) \rho_{1, k}(\lambda) g(\lambda) d \lambda .
\end{aligned}
$$

Now, repeating the argument in the proof of Proposition 2.6 and using Lemma 3.8 below, we obtain

Proposition 3.7.

$$
\text { (i ) } \rho_{1, k}(\lambda)=\left(\begin{array}{cccc}
\rho_{k, 1}(\lambda) & & & 0 \\
& \rho_{k, 2}(\lambda) & & \\
0 & & \ddots & \\
0 & & & \rho_{k, k}(\lambda)
\end{array}\right), \rho_{k, \nu}(\lambda) \in M_{2}^{++} \text {. }
$$


(ii) $\rho_{k, \nu}=n_{k, \nu} \rho_{k-1, \nu}$ for $1 \leq \nu<k$, where $n_{k, \nu}(\lambda)=\left\{\left(k^{2}-\nu^{2}\right)\left(4 k^{2}+\lambda^{2} / \nu^{2}\right)\right\}^{-1}$. Recall the definition of $\Psi_{k, 0}$ and $\rho_{k, 0}$ in (3.14), and put

$$
\begin{aligned}
& \Psi_{k}=\Psi_{0,0}(k=0) \quad \text { or } \quad\left(\Psi_{k, 0}, \Psi_{1, k}\right)(k>0), \\
& \rho_{k}=\rho_{0,0}(k=0) \quad \text { or } \quad \rho_{k, 0} \oplus \rho_{1, k}(k>0) .
\end{aligned}
$$

We can safely write $L^{2}\left(R, \rho_{k}\right)$ in place of $L^{2}\left(R^{*}, \rho_{k}\right)$. Keeping this remark in mind, let us define an onto isometry $\mathscr{F}_{k}: L^{2}(R)^{2 k+1} \rightarrow L^{2}\left(R, \rho_{k}\right)$ and its inverse $\mathscr{F}_{k}^{-1}$ as follows;

$$
\begin{aligned}
\mathscr{F}_{k} f(\lambda) & =\underset{N \rightarrow \infty}{\lim } \int_{|\tau|<N} \Psi_{k}^{*}(\tau, \lambda) f(\tau) d \tau, \\
\mathscr{F}_{k}^{-1} g(\lambda) & =\operatorname{li} \operatorname{i.m}_{N \rightarrow \infty} \int_{N-1<|\lambda|<N} \Psi_{k}(\tau, \lambda) \rho_{k}(\lambda) g(\lambda) d \lambda .
\end{aligned}
$$

By Propositions 3.2 and 3.7 and Lemma 3.4 one should find no difficulty in verifying that $\mathscr{F}_{k}$ and $\mathscr{F}_{k}^{-1}$ are well-defined and have presupposed properties. As to the estimates of solutions of the equations (3.15) and $\overline{(3.15)}$, we have

LEMMA 3.8.

(i) Fix $\lambda_{0} \in R^{*}$ and $\varepsilon>0$. Then there exist positives $\delta$ and $K$ such that

$$
\begin{aligned}
& \left|\check{\Phi}_{k}\left(\tau, \lambda_{0}\right)\right|<K \quad \text { on } \dot{D}_{\tau} \cap\{|\operatorname{Re} \tau| \geq 1\}, \\
& \left|\check{\Phi}_{k}(\tau, \lambda)\right|<K e^{\varepsilon|\tau|} \quad \text { on } R \times\left\{\left|\lambda-\lambda_{0}\right|<\delta\right\} .
\end{aligned}
$$

In particular the 0 -th row of $\Phi_{k}\left(\tau, \lambda_{0}\right)$ tends to zero as $\tau \rightarrow \pm \infty$.

(ii) Let $\chi_{k}(\tau, \lambda) \in M_{2 k+1,2 k}$ be a solution of $\overline{(3.15)}$ with $\check{\chi}_{k}(0, \lambda)=I_{2 k}$. Then (i) holds for $\chi_{k}$. See (3.3) for the definition of $\check{\Phi}_{k}$ and $\check{\chi}_{k}$.

(iii) Fix $\lambda_{0} \in\{|\operatorname{Im} \lambda|<1 / 4 \backslash\{0\}$. Then there exist positives $\delta$ and $K$ such that $\left|\Phi_{k}(\tau, \lambda)\right|<K e^{|\tau| / 8}$ on $R \times\left\{\left|\lambda-\lambda_{0}\right|<\delta\right\}$.

Proof. Note that $\check{\Phi}_{k}$ satisfies certain differential equation $\check{\Phi}_{k}^{\prime}=$ $V(\tau, \lambda) \check{\Phi}_{k}$. Hence the argument in the proof of Lemma 2.2 is available to prove (i) and (iii). The proof of (ii) is quite similar.

Q.E.D.

We intend to define closed subspaces $D_{k, \pm}^{e}$ of $L^{2}(R)^{2 k+1}$ by the aid of a fundamental system $\left\{\tilde{\psi}_{k, \nu} ; 1 \leq|\nu| \leq k\right\}$ of the equation $\overline{(3.15)}$;

$$
\tilde{\psi}_{k, \nu}=\bar{K}_{+, k-1} \tilde{\psi}_{k-1, \nu}(1 \leq|\nu|<k) \text { or } \tilde{\psi}_{k, \nu}(|\nu|=k),
$$

where the $k$-th row $\left(\tilde{\psi}_{k, \pm k}\right)_{k}$ satisfies $\overline{(3.19)}$ and is a holomorphic function on $\dot{D}_{\tau} \times C$ with the following form; 


$$
\begin{aligned}
& \left(\tilde{\psi}_{k, \pm k}\right)_{k}=\sigma^{\alpha k, \pm 1}\left(\sum_{n=0}^{\infty} \tilde{e}_{k, \pm k, n, k} \sigma^{n}\right), \tilde{e}_{k, \pm k, 0, k}=1 \text { if } \ell \neq-1 / 2 \text {, } \\
& \left(\tilde{\psi}_{k, k}\right)_{k}=\sigma^{\alpha k, 1}\left(\sum_{n=0}^{\infty} \tilde{e}_{k, k, n, k} \sigma^{n}\right), \quad \tilde{e}_{k, k, 0, k}=1 \\
& \left.\left(\tilde{\psi}_{k,-k}\right)_{k}=\left(\tilde{\psi}_{k, k}\right)_{k} \log \sigma+\sigma^{\alpha k, 1}\left(\sum_{n=1}^{\infty} \tilde{e}_{k,-k, n, k} \sigma^{n}\right)\right\} \text { if } \ell=-1 / 2 \text {. }
\end{aligned}
$$

Put $\tilde{\Psi}_{k, \nu}=\left(\tilde{\psi}_{k,-\nu}, \tilde{\psi}_{k, \nu}\right)(1 \leq \nu \leq k)$, and define $X_{k}(\lambda) \in M_{2}$ and $s_{k, \pm}(\lambda), r_{k, \pm}(\lambda)$ $\in M_{2,1}$ by

$$
\begin{aligned}
& \tilde{\Psi}_{k, k}(\tau, \lambda)=\bar{\Psi}_{k, k}(\tau, \lambda) X_{k}(\lambda) \quad \text { for }(\tau, \lambda) \in R \times R^{*}, \\
& s_{k, \pm}=X_{k} v_{ \pm}, \quad r_{k, \pm}=\left(\begin{array}{rr}
0 & 1 \\
-1 & 0
\end{array}\right) s_{k, \pm},
\end{aligned}
$$

where $v_{ \pm}={ }^{t}(1 \pm 1,1 \mp 1)$ or ${ }^{t}(0,2)$ according as $\ell \neq-1 / 2$ or not. Then recalling the definition of $s_{0, \pm}$ and $r_{0, \pm}$ in (3.9), set

$$
D_{k, \pm}^{e}=\mathscr{F}_{k}^{-1}\left\{\left(g_{\nu}\right) \in L^{2}\left(R, \rho_{k}\right) ;{ }^{t} \mathcal{S}_{\nu, \pm} g_{\nu}=0 \text { a.e. for } \nu=0,1, \cdots, k\right\} .
$$

We are now ready to state our main theorem in this section.

Theorem 3.1. Let $D_{k}$ be a closed subspace of $L^{2}(R)^{2 k+1}\left(k \in Z_{+}\right)$. Then the sequence $\left\{D_{k}\right\}_{k \in Z_{+}}$is a nontrivial one satisfying the conditions (Q.1) and (Q.2) iff it coincides with either $\left\{D_{k,-}^{\ell}\right\}$ or $\left\{D_{k,+}^{\ell}\right\}$ (see Theorem 2.2 for the definition of a nontrivial sequence).

For the proof we prepare some lemmas. Let $\left\{\zeta_{k, \nu}(\tau, \lambda) ; 1 \leq|\nu| \leq k\right\}$ and $\left\{\chi_{k, \nu}(\tau, \lambda) ; 1 \leq|\nu| \leq k\right\}$ be new fundamental systems of the equations (3.15) and (3.15) respectively, whose definition runs as follows.

$$
\zeta_{k, \nu}=K_{+, k-1} \zeta_{k-1, \nu}(1 \leq|\nu|<k) \quad \text { or } \quad \zeta_{k, \nu}(|\nu|=k),
$$

where the $k$-th component $\left(\zeta_{k, \pm k}\right)_{k}$, being holomorphic in $\dot{D}_{\tau} \times C$, assumes the form

$$
\begin{gathered}
\left(\zeta_{k, \pm k}\right)_{k}=\sigma^{\alpha k, \pm k}\left(\sum_{n=0}^{\infty} z_{k, \pm k, n, k} \sigma^{n}\right), \quad z_{k, \pm k, 0, k}=1 \text { if } \ell \neq-1 / 2, \\
\left(\zeta_{k, k}\right)_{k}=\sigma^{\alpha k, k}\left(\sum_{n=0}^{\infty} z_{k, k, n, k} \sigma^{n}\right), \quad z_{k, k, 0, k}=1 \\
\left.\left(\zeta_{k,-k}\right)_{k}=\left(\zeta_{k, k}\right)_{k} \log \sigma+\sigma^{\alpha k, k}\left(\sum_{n=1}^{\infty} z_{k,-k, n, k} \sigma^{n}\right)\right\} \text { if } \ell=-1 / 2 . \\
\chi_{k, \nu}=\bar{K}_{+, k-1} \chi_{k-1, \nu} \quad(1 \leq|\nu|<k) \text { or } \chi_{k, \nu}(|\nu|=k),
\end{gathered}
$$

where the $k$-th component $\left(\chi_{k, \pm k}\right)_{k}$, being holomorphic in $\dot{D}_{\tau} \times C$, has the form 


$$
\begin{aligned}
& \left(\chi_{k, \pm k}\right)_{k}=\sigma^{\alpha_{k, \pm k}}\left(\sum_{n=0}^{\infty} x_{k, \pm k, n, k} \sigma^{n}\right), \quad x_{k, \pm k, 0, k}=1 \text { if } \ell \neq-1 / 2, \\
& \left.\left(\chi_{k, k}\right)_{k}=\sigma^{\alpha, k}\left(\sum_{n=0}^{\infty} x_{k,-k, n, k} \sigma^{n}\right), \quad x_{k, k, 0, k}=1\right) \text { if } \ell=-1 / 2 \text {. } \\
& \left.\left(\chi_{k,-k}\right)_{k}=\left(\chi_{k, k}\right)_{k} \log \sigma+\sigma^{\alpha k, k}\left(\sum_{n=1}^{\infty} x_{k,-k, n, k} \sigma^{n}\right)\right\} \text { if } \ell=-1 / 2 \text {. }
\end{aligned}
$$

In view of (3.8), (3.16) and $\overline{(3.16)}, \zeta_{k, \nu}$ and $\chi_{k, \nu}(|\nu|=1 / 4,1, \cdots, k)$ have the following expression near $\sigma=0$; when $\ell \neq-1 / 2$,

$$
\zeta_{k, \nu}=\sigma^{\alpha k, \nu}\left(\sum_{n=0}^{\infty} z_{k, \nu, n} \sigma^{n}\right), \quad \chi_{k, \nu}=\sigma^{\alpha k, \nu}\left(\sum_{n=0}^{\infty} x_{k, \nu, n} \sigma^{n}\right),
$$

while in the case $\ell=-1 / 2, \zeta_{k, \nu}$ and $\chi_{k, \nu}(\nu>0)$ having the form as above, $\zeta_{k,-\nu}$ and $\chi_{k,-\nu}(\nu>0)$ have the following form

$$
\begin{aligned}
& \zeta_{k,-\nu}=\sigma^{\alpha k, \nu}\left(z_{k,-\nu, 0} \log \sigma+y_{k,-\nu, 0}+\sigma h(\sigma, \log \sigma)\right), \\
& \chi_{k,-\nu}=\sigma^{\alpha k, \nu}\left(x_{k,-\nu, 0} \log \sigma+\tilde{y}_{k,-\nu, 0}+\sigma h(\sigma, \log \sigma)\right) .
\end{aligned}
$$

We note that $z_{k,-\nu, 0}=z_{k, \nu, 0}$ and $x_{k,-\nu, 0}=x_{k, \nu, 0}$ if $\ell=-1 / 2$. The operator $R_{k}$ has been introduced just before Lemma 2.3 .

LEMMA 3.9.

$$
\text { (i) } R_{k} z_{k, \nu, n}=(-1)^{n} z_{k, \nu, n}, \quad R_{k} x_{k, \nu, n}=(-1)^{n} x_{k, \nu, n},
$$

where $\nu= \pm 1 / 4, \pm 1, \cdots, \pm k$ or $1 / 4,1, \cdots, k$ according as $\ell \neq-1 / 2$ or not.

(ii) $\left\langle{ }^{t} \chi_{k, \nu}(\tau, \lambda) G_{\alpha} \zeta_{k, \nu^{\prime}}(\tau, \xi)\right\rangle=0$ for $(\lambda, \xi) \in R^{*} \times R^{*}$ and $\alpha(\operatorname{Re} \alpha>0)$, if one of the following two conditions is satisfied;

$$
\begin{array}{lll}
\ell \neq-1 / 2, \quad \nu \nu^{\prime}<0, & \alpha_{k, \nu}+\alpha_{k, \nu^{\prime}} \geq-1, \\
\ell=-1 / 2, \quad \nu, \nu^{\prime}>0, & \alpha_{k, \nu}+\alpha_{k, \nu^{\prime}} \geq-1 .
\end{array}
$$

Of course $\lambda$ or $\xi$ should be positive according as $|\nu|=1 / 4$ or $\left|\nu^{\prime}\right|=1 / 4$.

Proof. Put $z_{k, \nu}(\sigma)=\sum_{n=0}^{\infty} z_{k, \nu, n} \sigma^{n}$ and $x_{k, \nu}=\sum_{n=0}^{\infty} x_{k, \nu, n} \sigma^{n}(0<\nu \leq k$ if $\ell=-1 / 2$ ). We shall show that

$$
R_{k} z_{k, \nu}(\sigma)=z_{k, \nu}(\sigma), \quad \overline{(3.34)} \quad R_{k} x_{k, \nu}=x_{k, \nu}(\sigma) .
$$

Only the proof of (3.34) for $\nu>0$ will be given. First, let $\nu=1 / 4$. Since $R_{k} L_{k}(\sigma) R_{k}=L_{k}(\sigma)$ by Lemma $2.3, R_{0} \zeta_{0, \nu} \propto \zeta_{0, \nu}$. In particular $R_{0} z_{0, \nu}(\sigma)=$ $c z_{0, \nu}(\sigma)$ for some constant $c$. Thus $c=1$, since $z_{0, \nu, 0} 1$. Suppose (3.34) is true up to $k$ for $\nu=1 / 4$. Keeping in mind that $R_{k+1} K_{+, k}(\sigma) R_{k}=$ $-K_{+, k}(\sigma)$, let $R_{k+1}$ operate to the both sides of $\zeta_{k+1, \nu}=K_{+, k} \zeta_{k, \nu}$ to obtain 
$(-1)^{\alpha_{k+1, \nu}} \sigma^{\alpha_{k+1, \nu}} R_{k+1} z_{k+1, \nu}(\sigma)=-(-1)^{\alpha k \nu} \zeta_{k+1, \nu}$. Therefore (3.34) holds for $\nu=1 / 4$. Secondly, let $\nu \geq 1$. Since $R_{k} M_{k}(\sigma) R_{k}=M_{k}(\sigma)$, there exists a constant $c_{1}$ such that $R_{1} z_{1,1}(\sigma)=c_{1} z_{1,1}(\sigma)$. As one can verify easily, $R_{1} z_{1,1,0}$ $=z_{1,1,0}$, which yields $c_{1}=1$. As in the case $\nu=1 / 4$, (3.34) with suffix $(k+1, \nu)$ holds if (3.34) is true. It remains, therefore, to prove that $R_{k} z_{k+1, k+1}(\sigma)=z_{k+1, k+1}(\sigma)$ under the condition that (3.34) is valid for any $\nu \geq 1$. Since $R_{k} K_{+, k}^{*}(\sigma) R_{k+1}=-K_{+, k}^{*}(\sigma)$, the equality $R_{k+1} z_{k+1, k+1}(\sigma)=$ $c_{k+1} z_{k+1, k+1}(\sigma)$, together with $K_{+, k}^{*} \zeta_{k+1, k+1} \propto \zeta_{k, k}$, yields $c_{k+1}(-1)^{\alpha_{k+1, k+1}} K_{+, k}^{*}$ $\times \zeta_{k+1, k+1}=-(-1)^{\alpha k, k} K_{+, k}^{*} \zeta_{k+1, k+1}$, namely $c_{k+1}=1$. This completes the proof of (i). (ii) To begin with, on an additional condition $\alpha_{k, \nu}+\alpha_{k, \nu^{\prime}}>$ $-1,{ }^{t} \chi_{k, \nu}(\tau, \lambda) \zeta_{k, \nu^{\prime}}(\tau, \xi)$ can be expanded as $\sum_{n=0}^{\infty} c_{n} \sigma^{2 n+1}$ by virtue of (i). Consequently the integral in question vanishes by Proposition 1.2 (i) [7]. Now we may assume that $\alpha_{k, \nu}+\alpha_{k, \nu^{\prime}}=-1$. Again by (i) it is enough to show that ${ }^{t} x_{k, \nu, 0} z_{k, \nu^{\prime}, 0}=0$. Note that $z_{k, \pm 1 / 4,0}$ and $x_{k, \pm 1 / 4,0}$ are proportional to $z_{k, \pm 1,0}$ and $x_{k, \pm 1,0}$ respectively, since so they are when $k=1$. Without loss of generality, let $|\nu|,\left|\nu^{\prime}\right| \geq 1$. It is clear that ${ }^{t} x_{k, \nu, 0} z_{k, \nu^{\prime}, 0}=0$ for $k=1$. Assume that this equality holds up to $k-1(k>1)$. Since $\alpha_{k,-k}+\alpha_{k, k}$ $=2 k-3$, either $\chi_{k, \nu}=\bar{K}_{+, k-1} \chi_{k-1, \nu}$ or $\zeta_{k, \nu^{\prime}}=K_{+, k-1} \zeta_{k-1, \nu^{\prime}}$. For the sake of definiteness suppose the latter is the case. Then by Lemma 3.10 below the coefficient of $\sigma^{-1}$ of ${ }^{t} \chi_{k, \nu} \zeta_{k, \nu^{\prime}}$ is equal to the corresponding one of ${ }^{t}\left({ }^{t} K_{+, k-1} \chi_{k, \nu}\right) \zeta_{k-1, \nu^{\prime}}$, which can be represented as ${ }^{t}\left(\sum_{\mu}^{\prime} a_{\mu} \chi_{k-1, \mu}\right) \zeta_{k-1, \nu^{\prime}}$. Here $\sum_{\mu}^{\prime}$ stands for $\sum_{\mu=\nu-1}^{-1}$ or $\sum_{\mu=\nu-1}^{k-1}$ according as $\nu<0$ or $\nu>0$. By the induction hypothesis ${ }^{t} x_{k, \nu, 0} z_{k, \nu^{\prime}, 0}=0$.

Q.E.D.

Lemma 3.10. Let $C$ and $D$ be constant matrices in $M_{p, q}$, and $x(\sigma)$ (resp. $z(\sigma)$ ) be $M_{p, 1}$ (resp. $M_{q, 1}$ )-valued functions of the form

$$
x(\sigma)=\sigma^{\alpha}\left(\sum_{n=0}^{\infty} x_{n} \sigma^{n}\right), \quad z(\sigma)=\sigma^{\beta}\left(\sum_{n=0}^{\infty} z_{n} \sigma^{n}\right) \text { with } \alpha+\beta=0 .
$$

Then the coefficient of $\sigma^{-1}$ of ${ }^{t} x(\sigma)\left(C d / d \sigma+D \sigma^{-1}\right) z(\sigma)$ is equal to the coefficient of $\sigma^{-1}$ of ${ }^{t}\left\{\left(-{ }^{t} C d / d \sigma+{ }^{t} D \sigma^{-1}\right) x(\sigma)\right\} z(\sigma)$.

Proof. This is because ${ }^{t} x_{0}(\beta C+D) z_{0}={ }^{t}\left\{\left(-\alpha^{t} C+{ }^{t} D\right) x_{0}\right\} z_{0}$. Q.E.D.

The next lemma is concerned with the if part of Theorem 3.1.

Lemma 3.11. Assume that, for any $(\lambda, \xi) \in R^{*} \times R^{*}, \alpha(\operatorname{Re} \alpha>0)$ and $\nu, \nu^{\prime}$ with either $\nu \nu^{\prime}<0$ or $\nu, \nu^{\prime}>0$ according as $\ell \neq-1 / 2$ or not, the following equality holds for any $k \leq k^{\prime}\left(\in Z_{+}\right)$;

$$
\left\langle{ }^{t} \chi_{k, \nu}(\tau, \lambda) G_{a} \zeta_{k, \nu}(\tau, \xi)\right\rangle=0 \text {. }
$$


(i) The equality (3.35) holds even for $k=k^{\prime}+1$.

(ii) $D_{k, \pm}^{\ell}\left(k \leq k^{\prime}\right)$ are invariant under the selfadjoint operators $L_{k}$ and $M_{k}$ and the semigroup $T_{t}(t \geq 0)$. In particular so are $D_{k^{\prime}+1, \pm}^{e}$ by (i).

To be precise, in (3.35) $\lambda$ or $\xi$ should be positive according as $|\nu|=1 / 4$ or $\left|\nu^{\prime}\right|=1 / 4$.

Proof. The proof is much like that of Lemma 2.8, and will be sketched briefly. (ii) Using relations $L_{k} \Psi_{k, 0}(\tau, \lambda)=\lambda \Psi_{k, 0}(\tau, \lambda)$ and $L_{k} \Psi_{k, \nu}(\tau, \lambda)$ $=\left\{\lambda^{2} /\left(4 \nu^{2}\right)-\nu^{2}\right\} \Psi_{k, \nu}(\tau, \lambda)$, we can show that $L_{k}$ and $M_{k}$ leave $D_{k, \pm}^{\ell}$ invariant. It remains to prove the $T_{t}$-invariance of $D_{k, \pm}^{\ell}(t \geq 0)$. By the assumption the integral $\left\langle{ }^{t} \tilde{\Psi}_{k, \nu}(\tau, \lambda) G_{\alpha} \Psi_{k, \nu^{\prime}}(\tau, \xi)\right\rangle\left(\nu, \nu^{\prime}=0, \cdots, k\right)$ takes the form $\left(\begin{array}{ll}* & 0 \\ 0 & *\end{array}\right)$ or $\left(\begin{array}{ll}* & * \\ * & 0\end{array}\right)$ according as $\ell \neq-1 / 2$ or not. Consequently a matrix $\hat{\rho}_{k, \nu}(\lambda)$ $=\rho_{k, \nu}(\lambda)^{t} X_{\nu}^{-1}(\lambda)$ turns out to be of the form $\left(\begin{array}{ll}* & 0 \\ 0 & *\end{array}\right)$ or $\left(\begin{array}{ll}0 & * \\ * & *\end{array}\right)$ according as $\ell \neq-1 / 2$ or not (cf. the proof of Theorem 1.1 [7]). Now it follows that, for any $h_{\nu^{\prime}} \in C_{0}\left(R^{*}\right)^{1}\left(C_{0}\left(R_{+}\right)^{1}\right.$ for $\left.\nu^{\prime}=0\right)$ and $\alpha(\operatorname{Re} \alpha>0)$, the integral

$$
{ }^{t} S_{\nu, \pm}(\lambda) \int \Psi_{k, \nu}^{*}(\tau, \lambda) G_{\alpha}\left(\int \Psi_{k, \nu^{\prime}}(\tau, \xi) \rho_{k, \nu^{\prime}}(\xi) r_{\nu^{\prime}, \pm}(\xi) h_{\nu^{\prime}}(\xi) d \xi\right) d \tau
$$

vanishes. This means that $G_{\alpha}$ sends a dense set of $D_{k, \pm}^{\ell}$ into $D_{k, \pm}^{\ell}$. In other words $T_{t}(t \geq 0)$ leaves $D_{k, \pm}^{\ell}$ invariant. (i) Thanks to Lemma 3.9, it suffices to prove $\left\langle{ }^{t} \chi_{k+1, \nu} G_{\alpha} \zeta_{k+1, \nu^{\prime}}\right\rangle=0$ on the additional condition that $\chi_{k+1, \nu}=\bar{K}_{+, k} \chi_{k, \nu}$ and $\zeta_{k+1, \nu}=K_{+, k} \zeta_{k, \nu^{\prime}}$. This can be done as in the proof of Lemma 2.8, since the exact analogues to (2.32) (2.35) hold. Q.E.D.

The following lemma is concerned with the only if part of Theorem 3.1 .

LEMMA 3.12.

(i) When $\ell \neq-1 / 2,{ }^{t} x_{k, \pm 1,0} z_{k, \pm 1,0} \neq 0$. If $\ell=-1 / 2$, then ${ }^{t} x_{k, \pm 1,0} \times$ $z_{k, \pm 1,0}=0$ while $\left({ }^{t} \tilde{y}_{k,-1,0} z_{k, 1,0}\right)\left({ }^{t} x_{k, 1,0} y_{k,-1,0}\right) \neq 0$.

(ii) For any $(\lambda, \xi) \in R^{*} \times R^{*}$ and $\nu, \nu^{\prime}$ with $\nu \nu^{\prime}>0$ or $\nu \nu^{\prime}<0$ according as $\ell \neq-1 / 2$ or not, there exists an $\alpha(\operatorname{Re} \alpha>0)$ such that $\left\langle{ }^{t} \tilde{\psi}_{k, \nu}(\tau, \lambda) G_{\alpha} \times\right.$ $\left.\psi_{k, \nu^{\prime}}(\tau, \xi)\right\rangle$ does not vanish. To be more precise, $\lambda$ or $\xi$ should be positive according as $|\nu|=1 / 4$ or $\left|\nu^{\prime}\right|=1 / 4$.

Proof. (i) We observed before that $z_{1, \pm 1 / 4,0} \propto z_{1, \pm 1,0}$ and $x_{1, \pm 1 / 4,0} \propto$ $x_{1, \pm 1,0}$. Let us define sequences $\left\{z_{k, \pm}\right\}_{k \in Z_{+}}$and $\left\{x_{k, \pm}\right\}_{k \in Z_{+}}$so that $z_{k, \pm}$ and $x_{k, \pm}$ are proportional to $z_{k, \pm 1,0}$ and $x_{k, \pm 1,0}$ respectively $(k>0)$ by the following recursion formulas; 


$$
\begin{aligned}
& z_{k+1, \pm}=\left[2\{-2 k-1 / 2 \pm(\ell+1 / 2)\} B_{k}-Y_{k}\right] z_{k, \pm}, \quad z_{0, \pm}=1, \\
& x_{k+1, \pm}=\left[-2\{-2 k-1 / 2 \pm(\ell \pm 1 / 2)\} B_{k}-\bar{Y}_{k}\right] x_{k, \pm}, \quad x_{0, \pm}=1 .
\end{aligned}
$$

Then, repeating the argument in the proof of (2.39), we obtain

$$
\begin{aligned}
& { }^{t} x_{k+1, \pm} A_{k}^{2} z_{k+1, \pm}=\frac{-\left(\alpha_{ \pm}-k\right)^{2}+k(k+1)+\ell(\ell+1)+1 / 4}{2+4\left(\alpha_{ \pm}-k+1 / 2\right)}{ }^{t} x_{k, \pm} z_{k, \pm}, \\
& { }^{t} x_{k+1, \pm} z_{k+1, \pm}=-8(k+1)(2 k+1)\left(\alpha_{ \pm}-k\right)\left(\alpha_{ \pm}-k-1 / 2\right)^{t} x_{k, \pm} z_{k, \pm},
\end{aligned}
$$

where $\alpha_{ \pm}= \pm(\ell+1 / 2)$. Now it is clear that ${ }^{t} x_{k, \pm} z_{k, \pm}=0(k>0)$ iff $\ell=$ $-1 / 2$. In case $\ell=-1 / 2$, let $\left\{y_{k}\right\}$ and $\left\{\tilde{y}_{k}\right\}$ be sequences defined by

$$
\begin{array}{ll}
y_{k+1}=\left\{+2(-2 k-1 / 2) B_{k}-Y_{k}\right\} y_{k}+2 B_{k} z_{k}, & y_{0}=0, \\
\tilde{y}_{k+1}=\left\{-2(-2 k-1 / 2) B_{k}-\bar{Y}_{k}\right\} \tilde{y}_{k}-2 B_{k} x_{k}, & \tilde{y}_{0}=0,
\end{array}
$$

where $z_{k}=z_{k, \pm}$ and $x_{k}=x_{k, \pm}$. Since $y_{k} \propto y_{k,-1,0}$ and $\tilde{y}_{k} \propto \tilde{y}_{k,-1,0}(k>0)$, it is enough to show the following relations (3.39) and (3.40).

$$
\begin{aligned}
& \left.\begin{array}{l}
{ }^{t} x_{k+1} y_{k+1}=-4 k(k+1)(2 k+1)^{2} x_{k} y_{k} \\
{ }^{t} \tilde{y}_{k+1} z_{k+1}=-4 k(k+1)(2 k+1)^{2}{ }^{t} \tilde{y}_{k} z_{k}
\end{array}\right\}(k>1) . \\
& \left({ }^{t} x_{k} y_{k}\right)\left({ }^{t} \tilde{y}_{k} z_{k}\right) \neq 0(k=1,2) \text {. }
\end{aligned}
$$

From now on we shall be concerned with ${ }^{t} \tilde{y}_{k} z_{k}$, for the same argument is applicable to ${ }^{t} x_{k} y_{k}$. By the definition of $\tilde{y}_{k}$ and $z_{k}$, we have

$$
\begin{aligned}
{ }^{t} \tilde{y}_{k+1} z_{k+1}= & { }^{t} \tilde{y}_{k}\left[-4\left\{(k+1)^{2}-A_{k}^{2}\right\}(2 k+1 / 2)^{2}\right. \\
& \left.+(4 k+1)\left(Y_{k}^{*} B_{k}-B_{k} Y_{k}\right)+Y_{k}^{*} Y_{k}\right] z_{k} \\
& -{ }^{t} x_{k}\left[-4\left\{(k+1)^{2}-A_{k}\right\}(2 k+1 / 2)-2 B_{k} Y_{k}\right] z_{k} .
\end{aligned}
$$

At this stage, notice that

$$
\begin{aligned}
& Y_{k}^{*} B_{k}-B_{k} Y_{k}=-(2 k+3) U_{k}, \quad B_{k} Y_{k}=(k+1) U_{k}+A_{k} V_{k}, \\
& Y_{k}^{*} Y_{k}=-V_{k}^{2}+4(k+1)(k+2) A_{k}-4(k+1)^{2} \ell(\ell+1) .
\end{aligned}
$$

Furthermore, $\boldsymbol{x}_{k}, \tilde{y}_{k}$ and $\boldsymbol{z}_{k}$ satisfy the following relations;

$$
\begin{aligned}
& \left\{2(-k+1 / 2) A_{k}-\bar{Y}_{k}\right\} \tilde{y}_{k}+2 A_{k} x_{k}=0, \\
& \left\{2(-k+1 / 2) A_{k}+V_{k}\right\} z_{k}=0, \\
& \left\{k^{2}+1 / 4+k(k+1)+\ell(\ell+1)-2 A_{k}^{2}+U_{k}\right\} z_{k}=0 .
\end{aligned}
$$

Indeed, (3.42) (3.44) follows from the equalities $\left(\bar{M}_{k}-\lambda\right) \chi_{k, 1}=0,\left(M_{k}-\lambda\right) \zeta_{k, 1}$ $=0$ and $\left(L_{k}-\lambda\right) \zeta_{k, 1 / 4}=0$ respectively. Since $U_{k}=A_{k} V_{k}-V_{k} A_{k}$ and since ${ }^{t} x_{k} A_{k}^{2} z_{k}=0(k>1)$ by (3.37), it follows from (3.42) and (3.43) that ${ }^{t} \tilde{y}_{k} U_{k} z_{k}$ 
$\propto{ }^{t} \tilde{y}_{k} A_{k}^{2} z_{k}$. Now (3.44) yields ${ }^{t} \tilde{y}_{k} A_{k}^{2} z_{k}=-k\{4(k-1)\}^{-1}{ }^{t} \tilde{y}_{k} z_{k}(k>1)$. Expressing the right side of (3.41) in terms of ${ }^{t} \tilde{y}_{k} z_{k}$ and ${ }^{t} \tilde{y}_{k} A_{k}^{2} z_{k}$, we get (3.39). Finally, it is not hard to verify (3.40) directly. (ii) We shall give the proof in the case $\ell=-1 / 2$. The other case is easier to deal with. For the sake of definiteness assume $\nu<0<\nu^{\prime}$. Then

$$
\tilde{\psi}_{k, \nu}=\sum_{\mu<0} \tilde{a}_{\mu} \chi_{k, \mu}, \quad \psi_{k, \nu^{\prime}}=\sum_{\mu>0} a_{\mu} \zeta_{k, \mu},
$$

where only one element of $\left\{\tilde{a}_{-1 / 4}, \tilde{a}_{-1}\right\}$ and $\left\{a_{1 / 4}, a_{1}\right\}$ vanishes. Therefore, in the neighborhood of $\sigma=0{ }^{t} \tilde{\psi}_{k, \nu} \psi_{k, \nu^{\prime}}$ takes the form $\sigma^{2 \alpha_{k, 1}}(\mathrm{c} \log \sigma+\sigma h(\sigma$, $\log \sigma))(c \neq 0)$ by virtue of (i). In particular $F(z)={ }^{t} \tilde{\psi}_{k, \nu}(\tau, \lambda) \psi_{k, \nu^{\prime}}(\tau, \xi) /$ $\sqrt{z(1-z)}$, as a function of $z=(1+i \operatorname{sh} \tau) / 2$, can not be holomorphic in $\{\operatorname{Re} z<1\}$ by Lemma 1.1 (ii) [7]. On the other hand, if $\left\langle{ }^{t} \tilde{\psi}_{k, \nu} G_{\alpha} \psi_{k, \nu^{\prime}}\right\rangle$ vanishes identically in $\{\operatorname{Re} \alpha>0\}$, it follows from Proposition 1.2 (ii) [7] that $F$ is holomorphic in $\{\operatorname{Re} z<1\}$, which is a desired contradiction.

We return to the

Q.E.D.

Proof of Theorem 3.1. We devide the proof into six parts as in the proof of Theorem 2.2. Since there arises no difficulty anew until the last step 6), it suffices to show that a nontrivial sequence $\left\{D_{k}\right\}$ satisfying the conditions (Q.1) and (Q.2) coincides with one of $\left\{D_{k,+}^{e}\right\}$. For the sake of definiteness, assume $\ell=-1 / 2 . \quad P_{k, \nu}(\nu=0,1, \cdots, k)$ now denotes the orthogonal projection: $L^{2}(R)^{2 k+1} \rightarrow\left\{\int \Psi_{k, \nu} \rho_{k, \nu} g d \lambda ; g \in L^{2}\left(R, \rho_{k, \nu}\right)\right\}$. By Proposition $3.1 D_{k}=D_{k,+}^{\ell}\left(=D_{k,-}^{\ell}\right)$ for $k=0$. We shall show that $D_{k}=D_{k,+}^{\ell}$ on the condition that $D_{k^{\prime}}=D_{k^{\prime},+}^{e}$ for any $k^{\prime}<k(k>0)$. Since $K_{+, k-1} D_{k-1,+}^{e}$ is dense in $\left(I-P_{k, k}\right) D_{k,+}^{\ell}$, the condition (Q.1) yields $\left(I-P_{k, k}\right) D_{k,+}^{\ell} \subset D_{k}$. In addition, by Lemmas 3.9 and 3.12 we have

$$
\begin{aligned}
& \left\langle{ }^{t} \tilde{\psi}_{k, k}(\tau, \lambda) G_{\alpha} \psi_{k, 1 / 4}(\tau, \xi)\right\rangle=0 \text { for any } \alpha(\operatorname{Re} \alpha>0), \\
& \left\langle{ }^{t} \tilde{\psi}_{k,-k}(\tau, \lambda) G_{\alpha} \psi_{k, 1 / 4}(\tau, \xi)\right\rangle \neq 0 \text { for some } \alpha^{\prime}\left(\operatorname{Re} \alpha^{\prime}>0\right) .
\end{aligned}
$$

These facts imply the existence of an element $f$ in $P_{k, 0} D_{k,+}^{\ell}$ such that $P_{k, k} G_{\alpha^{\prime}} f \neq 0$ for the above $\alpha^{\prime}$. Since $\mathscr{F}_{k} P_{k, k} G_{\alpha^{\prime}} f(\lambda)$ is anti-holomorphic in $\{|\operatorname{Im} \lambda|<1 / 4\} \mid\{0\}$ by Lemma 3.8 (iii), the closed linear span of $\left\{\left[\exp i t M_{k}\right]\right.$ $\left.\times P_{k, k} G_{\alpha^{\prime}} f ; t \in R\right\}$ coincides with $P_{k, k} D_{k,+}^{e}$. Now $D_{k,+}^{e} \subset D_{k}$ in view of the fact that $P_{k, k} G_{\alpha^{\prime}} f \in D_{k,+}^{\ell} \cap D_{k}$ and $\left(I-P_{k, k}\right) D_{k,+}^{\ell} \cap D_{k}$. As in the proof of Theorem 2.2, $D_{k}=P_{k, k} D_{k} \oplus\left(I-P_{k, k}\right) D_{k,+}^{e}$. To conclude the proof, we shall show $P_{k, k} D_{k}=P_{k, k} D_{k,+}^{\ell}$ by checking the following equality for $h$ in $P_{k, k} D_{k}$. 


$$
{ }^{t} S_{k,+}(\lambda) \int \Psi_{k, k}^{*}(\tau, \lambda) G_{\alpha} h(\tau) d \tau=0, \quad(\lambda, \alpha) \in R^{*} \times\{\operatorname{Re} \alpha>0\} .
$$

In fact, if the left side in (3.45) does not vanish for some $(\lambda, \alpha)$, then $P_{k, k} D_{k}=\left\{\int \Psi_{k, k} \rho_{k, k} g d \lambda ; g \in L^{2}\left(R, \rho_{k, k}\right)\right\}$ (see the proof of Theorem 2.2). Moreover, Lemma 3.12 ensures existence of an $\alpha^{\prime}\left(\operatorname{Re} \alpha^{\prime}>0\right)$ such that

$$
{ }^{t} S_{\nu,+}(\lambda)\left\langle\Psi_{k, \nu}^{*}(\tau, \lambda) G_{\alpha^{\prime}} \psi_{k,-k}(\tau, \xi)\right\rangle=\int{ }^{t} \tilde{\psi}_{k, \nu^{\prime}} G_{\alpha^{\prime}} \psi_{k,-k} d \tau \neq 0,
$$

where $\nu^{\prime}=1 / 4$ or $\nu$ according as $\nu=0$ or $\nu>0$. Using this fact, it can be easily shown that there is an $f$ in $P_{k, k} D_{k}$ such that $P_{k, \nu} G_{\alpha^{\prime}} f \notin P_{k, \nu} D_{k,+}^{\ell}$. This contradicts the decomposition $D_{k}=P_{k, k} D_{k} \oplus\left(I-P_{k, k}\right) D_{k,+}^{e}$. Hence (3.45) holds and we have proved that $D_{k}=D_{k,+}^{\ell}$.

Q.E.D.

The following lemma has been used in the proof of Lemma 3.3.

LEMMA 3.13. Let $f={ }^{t}\left(f_{k}, f_{k-1}, \cdots, f_{-k}\right)=\left(f_{\nu}\right)$ be absolutely continuous on $R$ with $f_{k} \in L^{2}(R)(k \geq 1)$. If $f$ satisfies

$$
M_{k} f=0 \quad \text { and } \quad K_{+, k-1}^{*} f=0,
$$

then $f=0$.

Proof. Thanks to the first equality in (3.46), we can represent $f_{k-1}$ and $f_{k-2}$ in terms of $f_{k}$ and its derivatives. Now the second equality yields a differential equation of $f_{k} ; f_{k}^{\prime \prime}+2 k$ th $\tau f_{k}^{\prime}-(k+\ell)(k-\ell-1) f_{k} / \operatorname{ch}^{2} \tau=0$. By the change of variable $z=(1+i \operatorname{sh} \tau) / 2, h(z)=f_{k}(\tau)$ satisfies

$$
h^{\prime \prime}+\frac{(2 k+1)(z-1 / 2)}{z(z-1)} h^{\prime}+\frac{(k+\ell)(k-\ell-1)}{\{2 z(z-1)\}^{2}} h=0 .
$$

Since the set of indicial roots at $z=0$ is $\{-(k+\ell) / 2,-(k-\ell-1) / 2\}$, a solution of (3.47) which is holomorphic in a punctured vicinity of $z=0$ is a trivial one. The set of indicial roots at $z=\infty$ is $\{0,2 k\}$. If $f_{k}=0$, then $f=0$ by the first equality in (3.46). To complete the proof, we shall show that $h$ is a nontrivial holomorphic function in a punctured vicinity of $z=0$ unless $f_{k}=0$. If $f_{k} \neq 0, h$ takes the form $z^{-2 k}\left(\sum_{n=0}^{\infty} c_{n} z^{-n}\right)\left(c_{0} \neq 0\right)$ in some region $\{|z|>K\}$. This is because $\int_{C}|h(z)|^{2} d|z| \mid \sqrt{z(1-z)}<\infty$ for $C=\{1 / 2+i y ; y \in R\}$. Since $h$ is continuous on the line $C$ by the assumption on $f_{k}, h$ is holomorphic in a punctured vicinity of $z=0$. Q.E.D.

At the end of this section we remark that 


$$
\begin{aligned}
K_{+, k-1}^{*} f & =\sum_{\nu=0}^{k-1} \int \Psi_{k-1, \nu} \rho_{k-1, \nu} r_{\nu, \pm} h_{\nu} d \lambda, \\
K_{+, k} f & =\sum_{\nu=0}^{k} \int \Psi_{k+1, \nu} \rho_{k+1, \nu} r_{\nu, \pm} n_{k+1, \nu}^{-1} h_{\nu} d \lambda,
\end{aligned}
$$

where

$$
f=\sum_{\nu=0}^{k} \int \Psi_{k, \nu} \rho_{k, \nu} r_{\nu, \pm} h_{\nu} d \lambda \quad\left(h_{\nu} \in C_{0}\left(R^{*}\right)^{1} \text { for } \nu=0, \cdots, k\right) .
$$

Indeed, (3.48) is clear by virtue of Propositions 3.2 and 3.7.

\section{§4. $P_{+}$-invariant subspaces for the representation $\left(U^{\ell, \epsilon}, \mathfrak{F}_{\mathcal{C}}^{\ell, \epsilon}\right)$}

In this section all $P_{+}$-invariant, closed proper subspaces in $\mathfrak{S}^{\ell, \varepsilon}$ will be determined. Throughout this section we assume $(\ell, \varepsilon) \neq(0,0)$. It has been established in $\S \S 2 \sim 3$ that the sequences $\left\{D_{k,+}^{\ell}\right\}_{k \in Z_{++\varepsilon}}$ satisfy the conditions (Q.1) and (Q.2) and that there are no other such nontrivial sequences (Theorems 2.2 and 3.1). Regarding $D_{k, \pm}^{\ell}$ as a subspace of $W_{k, \mu}^{\ell, s}=L^{2}(R)^{2 k+1}$, set

$$
\mathscr{D}_{ \pm}^{\ell, \varepsilon}=\sum_{k \in Z_{+}+\varepsilon} \sum_{\mu=-k}^{k} \oplus J_{k, \mu}^{\ell, s-1} D_{k, \pm}^{\ell},
$$

where $J_{k, \mu}^{\ell, \varepsilon}: \mathscr{W}_{k, \mu}^{\ell, \varepsilon} \rightarrow W_{k, \mu}^{\ell, \epsilon}$ is an onto isometry defined by (1.10). Theorem 4.1, together with Theorem 1.1, is our main result in this paper. As to the representation $\widetilde{S}_{m, \rho}$ of $G$, see $[12, \S 11]$.

Theorem 4.1. Let $\mathscr{D}$ be a closed proper subspace of $\mathfrak{S}^{\ell, \varepsilon}$. Then $\mathscr{D}$ is $P_{+}$-invariant iff it coincides with either $\mathscr{D}_{-}^{\ell, \varepsilon}$ or $\mathscr{D}_{+}^{\ell, \epsilon}$.

TheOREM 4.2. The representations of $S L(2, C)$ realized in $\mathscr{D}_{ \pm}^{\ell, \varepsilon}$ decompose into irreducible ones as

$$
\begin{gathered}
\sum_{n \in Z_{+}} \oplus \int_{R}^{\oplus} \mathbb{S}_{2 n+1, \rho} d \rho \quad \text { if } \varepsilon=1 / 2, \\
\int_{R_{+}}^{\oplus} \widetilde{S}_{0, \rho} d \rho \oplus \sum_{n-1 \in Z_{+}} \oplus \int_{R}^{\oplus} \widetilde{S}_{2 n, \rho} d \rho \quad \text { if } \varepsilon=0 .
\end{gathered}
$$

Remark. It is known [1] that the representation of $S L(2, C)$ in $\mathfrak{S}^{\ell, \varepsilon}$ is unitary equivalent to the 2-multiple of the representation (4.2) or (4.3) according as $\varepsilon=1 / 2$ or $\varepsilon=0$.

Proof of Theorem 4.1. The proof of the only if part is quite the same as that of the proof of Theorem 1.1. We shall, therefore, show that $\mathscr{D}_{ \pm}^{e, s}$ 
are $P_{+}$-invariant. Put $\mathscr{D}_{k, \mu, \pm}^{\ell, s}=J_{k, \mu}^{\ell, s-1} D_{k, \pm}^{\ell}$ and $\mathscr{D}_{k, \pm}^{\ell, \varepsilon}=\sum_{\mu=-k}^{k} \oplus \mathscr{D}_{k, \mu, \pm}^{\ell, \varepsilon}$. Then $\mathscr{D}_{k, \mu,+}^{\ell, \varepsilon}$ is invariant under $U^{\ell, \varepsilon}(t, 0,0,0, e)(t \geq 0)$ by Proposition 1.1 and Theorem 3.1. In addition $\mathscr{D}_{k, \pm}^{\ell, s}$ is $S U(2)$-invariant, for $H_{3}$ and $H_{-}$leave it invariant by (1.8). Therefore it is enough to show that $U^{\ell, \varepsilon}\left(0, \omega_{6}(t)\right)$ keeps $\mathscr{D}_{ \pm}^{e, \varepsilon}$ invariant. To this end put

$$
\begin{aligned}
& D_{k, \mu, \pm}^{\ell}=D_{k, \pm}^{\ell}, \quad \tilde{D}_{k, \mu, \pm}^{\ell}=\mathscr{F}_{k} D_{k, \pm}^{\ell} \\
& \hat{D}_{k, \mu}=D^{2}(R)^{k+1 / 2}\left(k \in Z_{+}+1 / 2\right) \text { or } L^{2}\left(R_{+}\right) \oplus L^{2}(R)^{k}\left(k \in Z_{+}\right) .
\end{aligned}
$$

Then a map $I_{ \pm, k, \mu}: \tilde{D}_{k, \mu, \pm}^{\ell} \rightarrow \hat{D}_{k, \mu}$ defined by

$$
I_{ \pm, k, \mu}\left(r_{\nu, \pm} h_{k, \mu, \nu}\right)=\left(h_{k, \mu, \nu} \sqrt{r_{\nu, \pm}^{*} \rho_{k, \nu} r_{\nu, \pm}}\right)
$$

is an onto isometry. Put further

$$
D_{ \pm}^{\ell, \varepsilon}=\sum_{k, \mu}^{\varepsilon} \oplus D_{k, \mu, \pm}^{\ell}, \quad \tilde{D}_{ \pm}^{\ell, \epsilon}=\sum_{k, \mu}^{\varepsilon} \oplus \tilde{D}_{k, \mu, \pm}^{\ell}, \quad \hat{D}^{\varepsilon}=\sum_{k, \mu}^{\varepsilon} \oplus \hat{D}_{k, \mu},
$$

where $\sum_{k, \mu}^{\varepsilon}=\sum_{k \in Z_{+}+\varepsilon} \sum_{\mu=-k}^{k}$. Now in terms of $\mathscr{F}_{k}$ and $I_{ \pm, k, \mu}$ we can define onto isometries $\mathscr{F}^{\ell, \varepsilon}: D_{ \pm}^{\ell, \varepsilon} \rightarrow \tilde{D}_{ \pm}^{\ell, s}$ and $I_{ \pm}^{\ell, \varepsilon}: \tilde{D}_{ \pm}^{\ell, s} \rightarrow \hat{D}^{e}$ in a trivial manner. Denote by $\hat{D}_{c}^{\varepsilon}$ a dense set $\left\{\left(\hat{h}_{k, \mu, \nu}\right) \in \hat{D}^{\varepsilon} ; \hat{h}_{k, \mu, \nu} \in C_{0}\left(R^{*}\right)^{1}\right.$ or $C_{0}\left(R_{+}\right)^{1}$ according as $\nu>0$ or $\nu=0, \hat{h}_{k, \mu, \nu}=0$ for sufficiently large $\left.k\right\}$. Then $\mathscr{D}_{ \pm, c}^{\ell, \varepsilon}=\left(I_{ \pm}^{\ell, \varepsilon} \mathscr{F}^{\ell, s}\right.$ $\left.\times J^{\ell, \varepsilon}\right)^{-1} \hat{D}_{c}^{\varepsilon}$ lies in the domain of $F_{3}$ by Lemma 1.2. Moreover, $F_{3} \mathscr{D}_{ \pm, c}^{\ell, \varepsilon} \subset$ $\mathscr{D}_{ \pm}^{\ell, \varepsilon}$ in virtue of Lemma $1.2,(2.45)$ and (3.48). To prove that $U^{\ell, \varepsilon}\left(0, \omega_{6}(t)\right)$ leaves $\mathscr{D}_{ \pm}^{\ell, \varepsilon}$ invariant, it is enough to show that $F_{3}$ restricted to $\mathscr{D}_{ \pm, c}^{\ell, \varepsilon}$ is essentially selfadjoint [7, Lemma 2.4]. To complete the proof we shall show that the image $\left(F_{3}-z\right) \mathscr{D}_{ \pm, c}^{\ell, \varepsilon}$ is dense in $\mathscr{D}_{ \pm}^{\ell, \varepsilon}$ for any $z(\operatorname{Im} z \neq 0)$. For this purpose, set

$$
\hat{F}_{3, \pm}=\left(I_{ \pm}^{\ell, \epsilon} \mathscr{F}^{\ell, \varepsilon} J^{\ell, \varepsilon}\right) F_{3}\left(I_{ \pm}^{\ell, \varepsilon} \mathscr{F}^{\ell, \nu} J^{\ell, \varepsilon}\right)^{-1} \text { restricted to } \hat{D}_{c}^{\varepsilon},
$$

and let us show that the image $\left(\hat{F}_{3, \pm}-z\right) \hat{D}_{c}^{\epsilon}$ is dense in $\hat{D}^{\epsilon}$. By virtue of Lemma 1.2, (2.45) and (3.48), we have the following relations for an $\hat{h}=\left(\hat{h}_{k^{\prime}, \mu^{\prime}, \nu^{\prime}}\right) \in \hat{D}_{c}^{\varepsilon}$ with $\hat{h}_{k^{\prime}, \mu^{\prime}, \nu^{\prime}}=0$ for $\left(k^{\prime}, \mu^{\prime}, \nu^{\prime}\right) \neq(k, \mu, \nu)$, where $k^{\prime}$ and $\nu^{\prime}$ run in $Z_{+}+\varepsilon$ and $\left\{\varepsilon, \varepsilon+1, \cdots, k^{\prime}\right\}$ respectively.

In case $\nu>0$,

$$
\begin{aligned}
\left(\hat{F}_{3} \hat{h}\right)_{k-1, \mu, \nu}(\lambda) & =\frac{\sqrt{((k \pm \mu))}}{k \sqrt{((2 k \pm 1))}}\left[\left\{k^{2}-\nu^{2}\right\}\left\{k^{2}+\left(\frac{\lambda}{2 \nu}\right)^{2}\right\}\right]^{1 / 2} \hat{h}_{k, \mu, \nu}(\lambda) \\
\left(\hat{F}_{3} \hat{h}\right)_{k, \mu, \nu}(\lambda) & =\frac{u \lambda}{2 k(k+1)} \hat{h}_{k, \mu, \nu}(\lambda)
\end{aligned}
$$




$$
\begin{aligned}
\left(\hat{F}_{3} \hat{h}\right)_{k+1, \mu, \nu}(\lambda)= & \frac{\sqrt{((k \pm \mu+1))}}{(k+1) \sqrt{((2 k+2 \pm 1))}}\left[\left\{(k+1)^{2}-\nu^{2}\right\}\right. \\
& \left.\times\left\{(k+1)^{2}+\left(\frac{\lambda}{2 \nu}\right)^{2}\right\}\right]^{1 / 2} \hat{h}_{k, \mu, \nu}(\lambda), \\
\left(\hat{F}_{3} \hat{h}\right)_{k^{\prime}, \mu^{\prime}, \nu^{\prime}}(\lambda)= & 0 \quad \text { otherwise. }
\end{aligned}
$$

In case $\nu=0$,

$$
\begin{aligned}
& \left(\hat{F}_{3} \hat{h}\right)_{k-1, \mu, 0}(\lambda)=\frac{\sqrt{((k \pm \mu))}}{\sqrt{((2 k \pm 1))}} \sqrt{k^{2}+\lambda} \hat{h}_{k, \mu, 0}(\lambda), \\
& \left(\hat{F}_{3} \hat{h}\right)_{k+1, \mu, 0}(\lambda)=\frac{\sqrt{((k \pm \mu+1))}}{\sqrt{((2 k+2 \pm 1))}} \sqrt{(k+1)^{2}+\lambda} \hat{h}_{k, \mu, 0}(\lambda), \\
& \left(\hat{F}_{3} \hat{h}\right)_{k^{\prime}, \mu^{\prime}, \nu^{\prime}}(\lambda)=0 \quad \text { otherwise. }
\end{aligned}
$$

In the above $\hat{F}_{3}=\hat{F}_{3, \pm}$. From now on we assume $\varepsilon=0$ for the sake of definiteness. Let $\hat{f}=\left(\hat{f}_{k, \mu, \nu}\right) \in \hat{D}^{\varepsilon}$ be orthogonal to the image $\left(\hat{F}_{3}-z\right) \hat{D}_{c}^{s}$. Then it can be easily seen that

$$
\begin{aligned}
& \frac{\sqrt{((k \pm \mu))}}{\sqrt{((2 k \pm 1))}} \sqrt{k^{2}+\lambda} \hat{f}_{k-1, \mu, 0}(\lambda)-z^{*} \hat{f}_{k, \mu, 0}(\lambda) \\
& \quad+\frac{\sqrt{((k \pm \mu+1))}}{\sqrt{((2 k+2 \pm 1))}} \sqrt{(k+1)^{2}+\lambda} \hat{f}_{k+1, \mu, 0}(\lambda)=0 \text { a.e. on } R_{+} \\
& \frac{\sqrt{((k \pm \mu))}}{k \sqrt{((2 k \pm 1))}}\left[\left\{k^{2}-\nu^{2}\right\}\left\{k^{2}+\left(\frac{\lambda}{2 \nu}\right)^{2}\right\}\right]^{1 / 2} \hat{f}_{k-1, \mu, \nu}(\lambda) \\
& \quad+\left(\frac{\mu \lambda}{2 k(k+1)}-z^{*}\right) \hat{f}_{k, \mu, \nu}(\lambda) \\
& \quad+\frac{\sqrt{((k \pm \mu+1))}}{(k+1) \sqrt{((2 k+2 \pm 1))}}\left[\left\{(k+1)^{2}-\nu^{2}\right\}\right. \\
& \left.\quad \times\left\{(k+1)^{2}+\left(\frac{\lambda}{2 \nu}\right)^{2}\right\}\right]^{1 / 2} \hat{f}_{k+1, \mu, \nu}(\lambda)=0 \text { a.e. on } R .
\end{aligned}
$$

Applying the corollary of Proposition 1.6 in the cases $(m, \rho)=(0,2 \sqrt{\lambda})$ and $(m, \rho)=(2 \nu, \lambda / \nu)$ to $(4.6)$ and (4.7) respectively, we obtain

$$
\hat{f}_{k, \mu, 0}(\lambda)=0 \text { a.e. on } R_{+} \text {, and } \hat{f}_{k, \mu, \nu}(\lambda)=0 \text { a.e. on } R(1 \leq \nu \leq k)
$$

respectively. This means that $\hat{f}=0$. We have shown that $F_{3}$ restricted to $\mathscr{D}_{ \pm, c}^{\ell, \varepsilon}$ are essentially selfadjoint in $\mathscr{D}_{ \pm}^{\ell, \varepsilon}$.

Q.E.D.

Proof of Theorem 4.2. Let $\mathscr{D}_{k, \mu, \pm}^{\ell, \varepsilon}$ be as in the proof of Theorem 4.1, 
and only the case $\varepsilon=0$ will be discussed. Note first that $\mathscr{D}_{k, k, \pm}^{\ell, \varepsilon}=\mathscr{W}_{k, k}^{\ell, s}$ $\cap \mathscr{D}_{ \pm}^{\ell, \epsilon}$ (see (1.7) for the definition of $\mathscr{W}_{k, k}^{\ell, \varepsilon}$ ). Moreover $\mathscr{D}_{k, k, \pm}^{\ell, \varepsilon} \ominus F_{+} \mathscr{D}_{k-1, k-1, \pm}^{\ell, \varepsilon}$ $=J_{k, k}^{\ell, \varepsilon}-1\left\{\int \Psi_{k, k} \rho_{k, k} r_{k, \pm} h ; r_{k, \pm} h \in L^{2}\left(R, \rho_{k, k}\right)\right\}$. In fact, the latter, say $J_{k, k}^{\ell, \varepsilon-1}$ $\times P_{k, k} D_{k, \pm}^{e}$, contains the former on account of (2.48) and (3.48), while the former contains the latter, because, for any $f \in H_{2}(R)^{2 k+1} \cap D_{k, \pm}^{e}$ and $h$ in $\mathscr{D}_{k-1, k-1, \pm}^{\ell, \varepsilon}$ as well as in the domain of $F_{+}$, we have

$$
\left\langle J_{k, k}^{\ell, \epsilon}-1 f, F_{+} h\right\rangle=\left\langle F_{-} J_{k, k}^{\ell, \varepsilon}-1 f, h\right\rangle \propto\left\langle K_{+, k-1}^{*} f, J_{k-1, k-1}^{\ell, \varepsilon} h\right\rangle=0,
$$

that is, because the former contains a dense subset $J_{k, k}^{\ell, \varepsilon-1}\left(H_{2}(R)^{2 k+1} \cap D_{k, \pm}^{\ell}\right)$ of $J_{k, k}^{\ell, s}-1 P_{k, k} D_{k, \pm}^{\ell}$. Now the following unitary equivalence relations are easy to verify.

$$
\begin{aligned}
& \Delta\left|\mathscr{D}_{0,0, \pm}^{\ell, \epsilon} \simeq L_{0, \ell}\right| D_{0, \pm}^{\ell} \simeq \int_{R_{+}}^{\oplus} \lambda d \lambda . \\
& \Delta^{\prime}\left|\left(\mathscr{D}_{k, k, \pm}^{\ell, \varepsilon} \ominus F_{+} \mathscr{D}_{k-1, k-1, \pm}^{\ell, s}\right) \simeq M_{k, \ell}\right| P_{k, k} D_{k, \pm}^{\ell} \simeq \int_{R}^{\oplus} \lambda d \lambda .
\end{aligned}
$$

In view of a general method to decompose a unitary representation of $G$ into irreducible ones, (4.8) and (4.9) means that the representations in $\mathscr{D}_{ \pm}^{\ell, \varepsilon}(\varepsilon=0)$ contain

$$
\int_{R_{+}}^{\oplus} \widetilde{S}_{0, \rho} d \rho \text { and } \int_{R}^{\oplus} \widetilde{S}_{2 k, \rho} d \rho
$$

respectively $[6, \S 3]$.

Q.E.D.

Acknowledgement. The author expresses his heart-felt thanks to Professors Hirai, Takenaka and Tatsuuma for their encouraging comments and advice. He thanks Mr. Itatsu for much fruitful discussion. It is also his great pleasure to extend his sincere thanks to Professors Hida, Kubo and Nomoto, who generously granted him the opportunity to study the subject.

\section{REFERENCES}

[1] E. Angelopoulos, Reduction on the Lorentz subgroup of UIR's of the Poincaré group induced by semisimple little group, Math. Phys., 15 (1974), 155-165.

[2] E. A. Coddington and N. Levinson, Theory of differential equations, McGraw-Hill, 1955.

[ 3 ] J. Diximier, $C^{*}$-algebras (English translation), North Holland, 1977.

[4] S. Itatsu and H. Kaneta, Spectral matrices for first and second order self-adjoint ordinary differential operators with long range potentials, Funkcialaj Ekvacioj, 24, no. 1 (1981), 23-45.

[5] - Spectral matrices for first and second order self-adjoint ordinary differen- 
tial operators with short range potentials, Funkcialaj Ekvacioj, 24, no. 2 (1981), $167-186$.

[6] H. Kaneta, Irreducibility of some unitary representations of the Poincaré group with respect to the Poincaré subsemigroup, I, Nagoya Math. J., 78 (1980), 113136.

[7] - Irreducibility of some unitary representations of the Poincare group with respect to the Poincaré subsemigroup, II, Nagoya Math. J., 87 (1982), 147-174.

[8] T. Kato, Perturbation theory for linear operators, Springer-Verlag, 1966.

[ 9 ] J. L. Lions and E. Magenes, Problémes aux limites non-homogénes et applications, Vol. 1, Dunod, 1968.

[10] G. M. Mackey, Induced representations of locally compact groups I, Ann. of Math., 55 (1952), 101-139.

[11] N. Mukunda, Zero-mass representations of the Poincaré group in an $O(3,1)$ basis, J. Math. Phys., 9 (1968), 532-536.

[12] M. A. Neumark, Lineare Darstellungen der Loretz gruppe, VED Deutsher Verlag: der Wissenshahten, 1963.

[13] N. Vilenkin, Special functions and the theory of group representations, AMS Translation Monographs 22, 1968.

[14] K. Yosida, Functional analysis, Springer-Verlag, 1965.

Department of Mathematics

Nagoya University

Current address :

Department of Mathematics

Faculty of Education

Tokushima University 